\title{
A rough surface damping contact model and its application in spatial variable stator vane mechanism with dry friction joint
}

\section{Shuai Cheng}

Shanghai Jiao Tong University School of Mechanical Engineering

Xianghui Meng ( $\nabla$ xhmeng@sjtu.edu.cn )

Shanghai Jiao Tong University School of Mechanical Engineering https://orcid.org/0000-0003-23871186

Rui Li

Shanghai Jiao Tong University School of Mechanical Engineering

Ruichao Liu

Shanghai Jiao Tong University School of Mechanical Engineering

\section{Rui Zhang}

Shanghai Jiao Tong University School of Mechanical Engineering

\section{Research Article}

Keywords: Rough surface, Contact damping, Spatial tribo-dynamics, Variable stator vane, Wear

Posted Date: March 11th, 2021

DOI: https://doi.org/10.21203/rs.3.rs-259039/v1

License: (c) (i) This work is licensed under a Creative Commons Attribution 4.0 International License. Read Full License 


\title{
Title
}

A rough surface damping contact model and its application in spatial variable stator vane mechanism with dry friction joint

\section{Author information}

- Shuai Cheng, Xianghui Meng*, Rui Li, Ruichao Liu, Rui Zhang

- School of Mechanical Engineering, Shanghai Jiao Tong University, Shanghai 200240, People's Republic of China

- Corresponding author: Xianghui Meng, School of Mechanical Engineering, Shanghai Jiao Tong University, No. 800, Dongchuan Road, Shanghai 200240, People's Republic of China, Email: xhmeng@sjtu.edu.cn

- ORCID: 0000-0003-2387-1186

\begin{abstract}
For the mechanical system without oil lubrication, the impact or collision often occurs in the joint clearance, such as the variable stator vane (VSV) mechanism. In the dry friction joint, the damping of the contact bodies has a significant effect on the simulation stability of the tribo-dynamics calculation process. In order to investigate the effect of contact damping and joint clearance on the VSV mechanism performance, this paper proposes a damping contact model on rough surfaces to calculate the clearance contact force between the trunnion and bushing, and the spatial tribodynamics of VSV is established by combining this model with spatial dynamics. In addition, the effect of clearance size on the tribo-dynamics is analyzed. The results show that the contact damping must be included in the contact force model of dry friction joints, otherwise the calculation process will oscillate or even not converge, but the contact damping effect can be ignored in the case of lubricating oil. The movement of the trunnion in the bushing is affected by the adjustment drive and the aerodynamic drag, which leads to the wear concentrated on the edge of the bushing. The clearance size affects the distribution of the damping forces and the rigid forces in the contact process, and the damping forces ensure the stability of the VSV tribo-dynamics simulation process. Moreover, with the increase of clearance, the adjustment accuracy
\end{abstract}


of the VSV mechanism is reduced, and the wear of the bushing is intensified.

\section{Keywords}

Rough surface $\cdot$ Contact damping $\cdot$ Spatial tribo-dynamics $\cdot$ Variable stator vane $\cdot$ Wear

\section{Declarations}

\section{- $\quad$ Funding}

This study was supported by the National Natural Science Foundation of China (Grant No. 51875344), the Industry-University-Research cooperation project in Minhang District of Shanghai (Grant No. 2019MHC050), and the Shanghai Science and Technology Committee (Grant No. 19511133000).

\section{- Conflicts of interest/Competing interests}

The authors declare that they have no conflict of interest.

- Availability of data and material (Not applicable)

- Code availability (Not applicable)

- Authors' contributions

All authors contributed to the study conception and design. Shuai Cheng built the models, carried out simulation analysis and wrote the first draft; Xianghui Meng proposed improvements to the models and the paper. Models analysis and verification were carried out by Rui Li, Ruichao Liu and Rui Zhang. All authors read and approved the final manuscript.

- Ethics approval

Compliance with ethical standards

\section{- Consent to participate}

The authors consent to participate

\section{- Consent for publication}

The authors agree to publish 


\title{
A rough surface damping contact model and its application in spatial variable stator vane mechanism with dry friction joint
}

\author{
Shuai Cheng, Xianghui Meng*, Rui Li, Ruichao Liu, Rui Zhang
}

\begin{abstract}
For the mechanical system without oil lubrication, the impact or collision often occurs in the joint clearance, such as the variable stator vane (VSV) mechanism. In the dry friction joint, the damping of the contact bodies has a significant effect on the simulation stability of the tribo-dynamics calculation process. In order to investigate the effect of contact damping and joint clearance on the VSV mechanism performance, this paper proposes a damping contact model on rough surfaces to calculate the clearance contact force between the trunnion and bushing, and the spatial tribodynamics of VSV is established by combining this model with spatial dynamics. In addition, the effect of clearance size on the tribo-dynamics is analyzed. The results show that the contact damping must be included in the contact force model of dry friction joints, otherwise the calculation process will oscillate or even not converge, but the contact damping effect can be ignored in the case of lubricating oil. The movement of the trunnion in the bushing is affected by the adjustment drive and the aerodynamic drag, which leads to the wear concentrated on the edge of the bushing. The clearance size affects the distribution of the damping forces and the rigid forces in the contact process, and the damping forces ensure the stability of the VSV tribo-dynamics
\end{abstract}

S. Cheng $\cdot$ R. Li $\cdot$ R.C. Liu $\cdot$ R. Zhang

School of Mechanical Engineering, Shanghai Jiao Tong University, Shanghai 200240, People's

Republic of China

Email: Ashuai@sjtu.edu.cn

${ }^{*}$ Corresponding author: X.H. Meng

School of Mechanical Engineering, Shanghai Jiao Tong University, No. 800, Dongchuan Road, Shanghai 200240, People's Republic of China.

Email: xhmeng@sjtu.edu.cn 
simulation process. Moreover, with the increase of clearance, the adjustment accuracy of the VSV mechanism is reduced, and the wear of the bushing is intensified.

Keywords : Rough surface $\cdot$ Contact damping $\cdot$ Spatial tribo-dynamics $\cdot$ Variable stator vane $\cdot$ Wear

\section{Introduction}

It is inevitable that there are clearances in the joints of the mechanism, which will cause motion error, vibration, wear, and even reduce the overall performance of the system. In order to study the mechanical structure with clearance, it is essential to establish a reasonable and accurate contact model, especially in the dry friction joint, there is no lubricating medium to provide buffer, and the intense impact or collision often occurs. In this case, if the contact damping of the friction pair material is not considered, the calculation of the contact force will oscillate, and even lead to the nonconvergence of the dynamic simulation process.

Most contact models are based on Hertz contact model, which only study the effect of material properties and geometric features on the contact force, without considering the hysteresis damping of material. Goldsmith [1] used linear spring and linear damping model to describe the collision process, which laid the foundation for the contact model with damping, namely Kelvin-Voigt model. After that, many scholars optimized the linear spring damping model [2-4], in which Lankarani and Nikravesh [4] deduced the damping coefficient through the momentum principle in the contact process and proposed a contact force model with hysteresis damping, namely the LN model. The model not only considered the effect of material properties and geometric features on the contact force, but also reflected the influence of motion state on the collision process, so it has been widely used. For example, Zhao et al. [5], Chen et al. [6], Li et al. [7] and Sun et al. [8] used the LN model to calculate the normal contact force of the clearance joints to study the dynamics of the planar mechanical system. Yan [9] and Xiang [10] calculated the contact force at the clearance joints based on the LN model to study the dynamics of the spatial linkage mechanism. The LN model is suitable for the contact 
of two spheres or cylinders, or the contact of they with a flat plate [11], these research objects are nonconformal contact. However, for the joints with conformal contact, the contact model should be based on the surface-to-surface contact, and in this case, the influence of surface topography cannot be ignored.

On the microscopic scale, the contact between two surfaces is actually the contact of asperities [12,13], and the contact area depends on the surface morphology, so the accurate contact model should be built on the rough surface. Greenwood and Williamson [12] used statistical method and Hertz contact theory to calculate the contact force between rough surface and rigid flat. Later, Greenwood and Tripp [13] obtained the contact calculation methods of rough surfaces, known as the GT model. Moreover, they proposed that two-rough-surface contact can be equal to equivalent single-rough-surface contact. Chang [14] proposed that the contact sphere transited from the elastic to the elastic-plastic regime after the contact interference reached the critical value. Kogut and Etsion [15] established an elastoplastic contact force model for rough surface contact namely the KE model, and clearly distinguished the elastoplastic transition regime according to critical interference. Unfortunately, these excellent contact models did not consider the damping of asperities. Chen et al. [16] and Pan et al. [17] obtained the damping dissipation factor of rough surface in the contact process by fractal method, and derived the dimensionless contact damping from the damping dissipation factor, but the dimensionless damping could not be applied to the calculation of actual contact force. Through the above studies, it can be found that using statistical model to extend the hysteresis damping of a single asperity to the whole surface is a feasible method to establish the contact damping model of rough surface. Based on this idea, this paper established a rough surface damping contact (RSDC) model and applied it to the tribo-dynamics investigation of the variable stator vane (VSV) mechanism.

The VSV mechanism of compressor is the most important motion adjusting mechanism in the engine, which can prevent the compressor from surging and effectively improve the overall efficiency of the engine by changing the vane angle to adjust the incoming angle of attack, and is widely used in modern aeroengines [18-20]. 
After long-term operation of the VSV mechanism, the rotation of the vane trunnion will inevitably wear the bushing, resulting in the gradual increase of the assembly clearance, which directly affects the motion accuracy and reliability of the mechanism. Therefore, it is of great significance to study the contact and wear of key joints of the VSV mechanism for the development of aeroengine. However, the VSV mechanism runs in a high temperature environment of more than $400{ }^{\circ} \mathrm{C}$, which makes the joints unable to use the lubricating medium but in dry friction conditions. As mentioned earlier, in this case, the RSDC model must be used to calculate the contact force at the joints. In addition, the bushing of VSV mechanism is generally made of polymer materials to protect the components [21], which means that the contact force of the bushing contains a more significant damping effect. It should also be noted that the trunnion rotates relative to the bushing, the tangential force is much greater than the static friction, and the tangential damping can be ignored [22,23].

The difficulty in the study of tribo-dynamics of the VSV mechanism is that it is a spatial mechanism, which leads to the interaction of clearance friction pairs is threedimensional. Therefore, the accurate analysis model of the mechanism should also be based on the spatial dimension. Compared with planar mechanism, the dynamics formulas of spatial mechanism are more various and complex. Take a torsion rod type VSV mechanism as an example, which adopts spatial multi-rod linkage to adjust vane angle, and the overall structure diagram is shown in Figure 1. The actuator drives the crank to rotate, the crank drives the drag link, and then the drag link drives the unison ring to make circular motion. Driven by the unison ring, the lever arm rotates around the axis of the vane trunnion to adjust the vane. During the process of adjustment, the rotation of the lever arm will cause the axial movement of the unison ring and make the drag link rotate in the spatial dimension. It can be seen that the whole mechanism has obvious spatial motion. Yu et al. [24] simplified the VSV mechanism to a planar mechanism for kinematic analysis, which ignored the transmission of motion in the three-dimensional space, so there were errors in the simulation results. Some scholars analyzed the overall structure of the VSV in the spatial dimension. Reitenbach et al. [25] took the engine fuel consumption as the objective function and designed the 
optimal setting scheme of stator vane through the aero engine simulation software tool Gas Turbine Laboratory. Wang et al. [26] studied the influence of the penny platform locations on the gas flow through experiments and numerical simulation, and proposed that optimizing the structure and size of the VSV can improve the aerodynamic performance. Tang et al. [27] combined forward and inverse kinematics design method to optimize the global dimensional scale of the VSV mechanism to improve the accuracy of the vane rotation angle. And Riesland [28] used the ADAMS software to simulate the dynamics of the VSV mechanism. However, these researches did not consider the effect of the clearance in the joints on the system, and could not describe the nonlinear dynamics of the VSV mechanism in detail. Therefore, the reasonable tribo-dynamics of the mechanism should be based on the RSDC model on the one hand, and on the other hand, it should be built in the three-dimensional space.

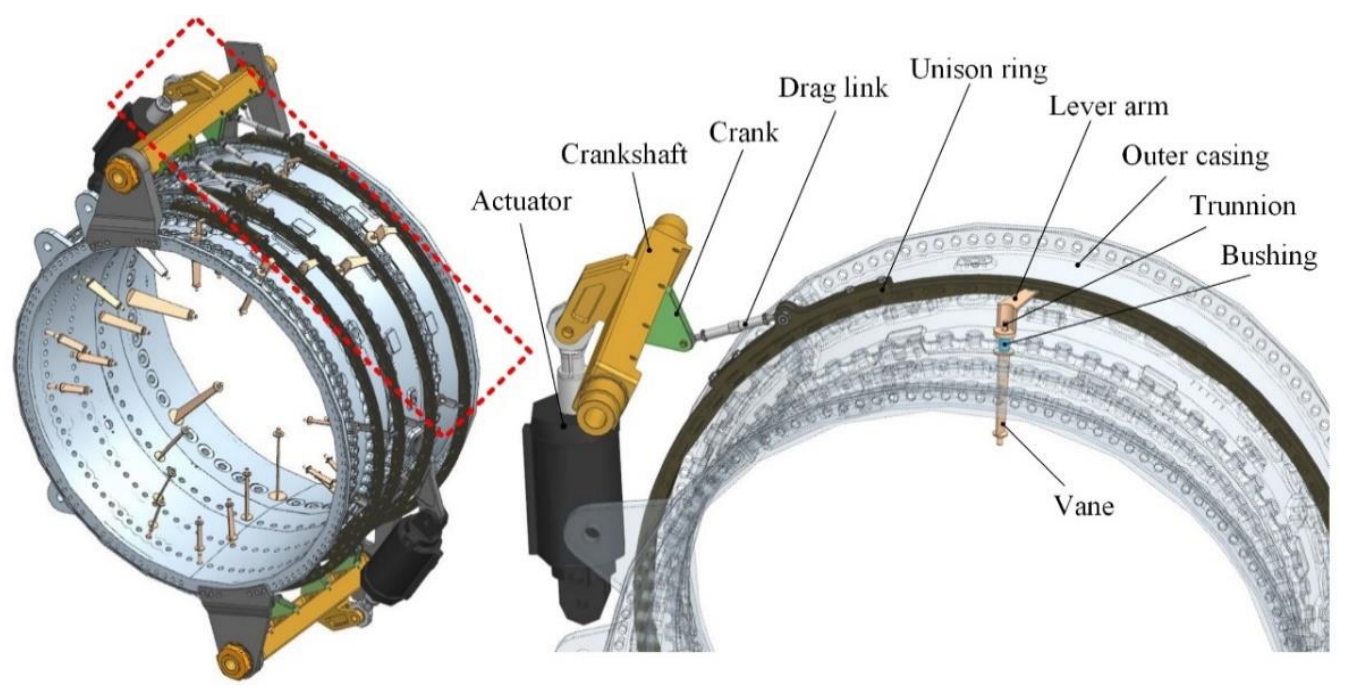

Fig 1 Schematic diagram of the VSV mechanism

In this paper, a rough surface damping contact model is established, and the dynamics simulation of journal bearing is carried out to verify the effect of the model under the condition of dry friction and lubrication. The rough surface damping contact model is applied to the calculate the clearance contact forces between the trunnion and bushing of the VSV mechanism, the kinematics and the dynamics are solved by the spatial coordinate system and the Newton-Euler equation respectively, and then the spatial tribo-dynamics of the VSV is established. Based on the spatial tribo-dynamics, 
the effect of clearance size on the system is studied and analyzed. In view of the symmetry of the VSV mechanism, the research on single-stage adjusting structure is still representative.

\section{Mathematical model}

\subsection{Rough surface damping contact model}

In this section, the damping characteristics of asperities are considered in the study of surface contact, and a rough surface damping contact (RSDC) model is proposed, the expression of contact pressure is as follows:

$$
P_{c}(\delta, \dot{\delta})=P_{k}(\delta)+P_{d}(\dot{\delta})
$$

Where the contact pressure $P_{c}$ is composed of rigid pressure $P_{k}$ and damping pressure $P_{d}, \delta$ and $\dot{\delta}$ represent the relative interference and the interference velocity of the asperities, respectively. The elastic-plastic contact model proposed by Kogut and Etsion is used to obtain the rigid pressure $P_{k}$. According to the KE model, the contact process is divided into elastic stage, elastic-plastic stage and plastic contact stage by the critical interference $\delta_{c}$, then $P_{k}$ can be expressed as [29,30]:

$$
P_{k}(\delta)=P_{e}+P_{e p 1}+P_{e p 2}+P_{p}
$$

where $P_{e}$ is the elastic contact pressure, $P_{e p 1}$ and $P_{e p 2}$ are the elastic-plastic contact pressure, and $P_{p}$ is the plastic contact pressure, specifically expressed as:

$$
\begin{gathered}
P_{e}\left(h^{*}\right)=\frac{2}{3} K H \pi(\xi \beta \sigma)\left[\delta_{c}^{*-0.5} \int_{h^{*}}^{h^{*}+\delta_{c}^{*}} \delta^{* 1.5} \Phi\left(z^{*}\right) d z^{*}\right] \text { for } \delta<\delta_{c} \\
P_{e p 1}\left(h^{*}\right)=\frac{2}{3} K H \pi(\xi \beta \sigma)\left[1.03 \delta_{c}^{*-0.425} \int_{h^{*}+\delta_{c}^{*}}^{h^{*}+6 \delta^{*}} \delta^{* 1.425} \Phi\left(z^{*}\right) d z^{*}\right] \text { for } \delta_{c}<\delta<6 \delta_{c} \\
P_{e p 2}\left(h^{*}\right)=\frac{2}{3} K H \pi(\xi \beta \sigma)\left[1.4 \delta_{c}^{*-0.263} \int_{h^{*}+6 \delta_{c}^{*}}^{h^{*}+110 \delta_{c}^{*}} \delta^{* 1.263} \Phi\left(z^{*}\right) d z^{*}\right] \text { for } 6 \delta_{c}<\delta<110 \delta_{c} \\
P_{p}\left(h^{*}\right)=2 H \pi(\xi \beta \sigma) \int_{h^{*}+110 \delta_{c}^{*}}^{\infty} \delta^{* 1} \Phi\left(z^{*}\right) d z^{*} \text { for } \delta>110 \delta_{c}
\end{gathered}
$$

where $H$ is the hardness of the softer material, $K$ is the hardness coefficient can be obtained by $K=0.454+0.41 v$, and $v$ is the Poisson's ratio of the softer material. $\xi$ is the equivalent areal asperity density, $\beta$ is the equivalent curvature radius of asperity, $\sigma$ is the equivalent standard deviation of surface height. $E$ is the equivalent elastic 
modulus, $E=1 /\left[\frac{1-v_{1}^{2}}{E_{1}}+\frac{1-v_{2}^{2}}{E_{2}}\right]$, where $E_{1}, E_{2}$ and $\nu_{1}, v_{2}$ are the elastic modulus and Poisson ratios of the contact surfaces, respectively. $\Phi\left(\mathrm{z}^{*}\right)$ is the asperity height probability density function. $\delta^{*}=z^{*}-h^{*}, z^{*}$ and $h^{*}$ are the dimensionless asperity height and separation of surfaces respectively. $\delta_{c}$ and its dimensionless form $\delta_{c}{ }^{*}$ can be expressed as:

$$
\left\{\begin{array}{l}
\delta_{c}=\left(\frac{\pi K H}{2 E}\right)^{2} \beta \\
\delta_{c}^{*}=\left(\frac{\pi K H}{2 E}\right)^{2} \frac{\beta}{\sigma}
\end{array}\right.
$$

Lankarani and Nikravesh established a contact force model with hysteresis damping. For two spheres with an equivalent radius of $R$, the contact force $F_{n}$ follows the relation according to the $\mathrm{LN}$ model:

$$
F_{n}=K_{n} \delta^{1.5}+D_{n} \dot{\delta}
$$

where $K_{n}=\frac{4}{3} E \sqrt{R}, D_{n}$ is the damping coefficient, $D_{n}=\frac{E \sqrt{R}\left(1-e^{2}\right) \delta^{1.5}}{\dot{\delta}_{-}} . e$ is the coefficient of restitution and defined as $e=-\frac{\dot{\delta}_{+}}{\dot{\delta}_{-}}, \dot{\delta}_{-}$and $\dot{\delta}_{+}$represent the velocity of initial contact and separation, respectively.

Extend the contact force of the two spheres to the rough surfaces covered with asperities, and then the damping pressure $P_{d}$ of the surfaces with Gaussian distribution of asperities is obtained:

$$
P_{d}(\dot{\delta})=\frac{E\left(\xi \sqrt{\beta} \sigma^{1.5}\right)\left(1-e^{2}\right) \int_{h^{*}}^{\infty} \delta^{* 1.5} \Phi\left(z^{*}\right) d z^{*}}{\dot{\delta}_{-}} \dot{\delta}
$$

In the LN model, usually given a value of restitution coefficient $e$ to calculate the contact force. However, $e$ depends on the hysteresis characteristic of material, so it is necessary to clarify the value of $e$. The damping loss factor $\mu$ can describe the hysteresis characteristic, and it can be expressed by the ratio of the dissipated energy $W_{p}$ and the elastic strain energy $W_{e}$ during the entire contact process:

$$
\mu=\frac{W_{p}}{W_{e}}
$$

where $W_{e}$ and $W_{p}$ can be obtained from the work done by the elastic force and the plastic force in the KE model, respectively. Then $W_{e}$ and $W_{p}$ on the unit area $A_{n}$ can be expressed as [17]: 


$$
\left\{\begin{array}{l}
W_{e}=A_{n} \int_{0}^{\delta}\left(P_{e}+P_{e p 1}+P_{e p 2}\right) d \delta \\
W_{p}=A_{n} \int_{0}^{\delta}\left(P_{p}+P_{e p 2}+P_{e p 1}\right) d \delta
\end{array}\right.
$$

In addition, the dissipated energy and the elastic strain energy are respectively equal to the consumption of kinetic energy and the residual kinetic energy during the contact process, so the damping loss factor $\mu$ can also be expressed by the following formula:

$$
\mu=\frac{\frac{1}{2} m \dot{\delta}_{-}{ }^{2}-\frac{1}{2} m \dot{\delta}_{+}{ }^{2}}{\frac{1}{2} m \dot{\delta}_{+}{ }^{2}}=\left(\frac{\dot{\delta}_{-}}{\dot{\delta}_{+}}\right)^{2}-1=\left(\frac{1}{e}\right)^{2}-1
$$

Then the coefficient of restitution $e$ can be obtained from the damping loss factor $\mu$, that is, $e=\frac{1}{\sqrt{\mu+1}}$. Therefore, the damping pressure $P_{d}$ on the rough surfaces can be modified as:

$$
P_{d}(\dot{\delta})=\frac{E\left(\xi \sqrt{\beta} \sigma^{1.5}\right) \mu \int_{h^{*}}^{\infty} \delta^{* 1.5} \Phi\left(z^{*}\right) d z^{*}}{(\mu+1) \dot{\delta}_{-}} \dot{\delta}
$$

By introducing Eqs. (2) and (10) into Eq. (1), the RSDC model is obtained. The application object of this model is no longer point or line contact, but surface contact. Therefore, the RSDC model should be more accurate in the study of conformal contact friction pairs.

\subsection{Spatial kinematics model of the VSV mechanism}

The single-stage VSV with one vane can be simplified as a spatial multi-rod mechanism, as shown in Figure 2. The rod $1\left(A S_{l}\right)$ is the driving crank, which is connected with the casing through the rotary joint $A$, and the rod $2\left(S_{1} S_{2}\right)$ is the drag link, which is connected with the rod 1 and the unison ring (rod 3) through the spherical joints $S_{1}$ and $S_{2}$ respectively. The rod 3 is composed of $S_{1} B$ and $B C$ which are fixedly connected. One end of the rod 3 is connected with the outer casing through the cylindrical joint $B$, and the other end is connected with the lever arm rod $4\left(S_{3} E\right)$ through the pin $(C D)$ and the spherical joint $S_{3}$, wherein $S_{3}$ can move along the axis of the pin. Finally, the rod 4 is fixedly connected with the vane, the trunnion of the vane is connected with the casing through the rotary joint $E$, and the bushing is between the trunnion and the casing. 
Regarding all joints as ideal pairs, the degree of freedom of the mechanism can be obtained by the G-K formula:

$$
M=d(n-g-1)+\sum_{i=1}^{g} f_{i}+v-\xi
$$

Where $M$ is the degree of freedom of the mechanism, $d$ is the common constraint, $n$ is the number of components (including the frame), $g$ is the number of kinematic pairs, $f_{i}$ is the degree of freedom of joint $i, v$ is the number of redundant constraints, and $\xi$ is the number of local degrees of freedom. In this mechanism, $d=6, n=5, g=6, f_{l}=1$, $f_{2}=3, f_{3}=3, f_{4}=2, f_{5}=4, f_{6}=1, v=0, \xi=1$, so $M=1$.

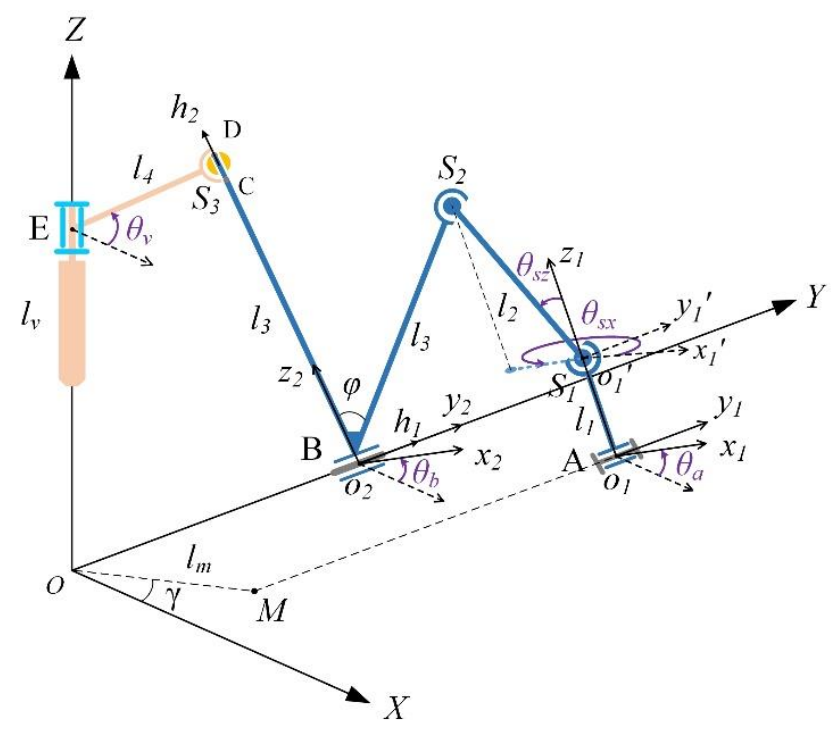

Fig 2 Schematic diagram of spatial multi-rod mechanism

In Figure 2, the global coordinate system $O-X Y Z$ is established, in which the origin is at point $O$, the $Y$-axis is along the casing axis, the $Z$-axis is vertically upward along the trunnion axis, and the $X$-axis is perpendicular to the $O Y Z$ plane. The local coordinate system $o_{1}-x_{1} y_{1} z_{1}$ takes point $o_{1}$ as the origin, the $z_{1}$-axis is fixedly connected to rod 1 , the $y_{1}$-axis is along the axis of the casing, and the $x_{1}$-axis is perpendicular to the $o_{1} y_{1} z_{1}$ plane. The local coordinate system $o_{2}-x_{2} y_{2} z_{2}$ takes point $o_{2}$ as the origin, and the $z_{2}$-axis is fixed on the $B C$ rod, the $y_{2}$-axis is along the axis of the casing, and the $x_{2}$-axis is perpendicular to the $o_{2} y_{2} z_{2}$ plane.

During the operation of the VSV mechanism, the rod 1 rotates only around the $y_{1^{-}}$ axis, the angle is $\theta_{a}$, the rod 2 rotates and moves in space, and the angle with the $z_{1^{-}}$- 
axis is $\theta_{S Z}$, and the angle between its projection on the $o_{1}{ }^{\prime} x_{1} y_{1}^{\prime}$ plane and $x_{1}{ }^{\prime}$-axis is $\theta_{s x}$, where $o_{1}^{\prime} x_{1} y_{1}$ is the plane that $o_{1} x_{1} y_{1}$ translation along the $z_{1}$-axis. The rod 3 rotates around $y_{2}$-axis with an angle of $\theta_{b}$ and moves along $y_{2}$-axis with a distance of $h_{1}$. The moving distance of the spherical joint $S_{3}$ on the pin is $h_{2}$ The rod 4 and the vane rotate synchronously with an angle of $\theta_{v}$. The attitudes of components in the initial position are: $\theta_{a}=-16^{\circ}, \theta_{s x}=0, \theta_{b}=0, \theta_{v}=90^{\circ}, h_{1}=0, h_{2}=0$, and the direction of coordinates and motion marked in the Figure 2 are positive.

According to the motion characteristics of the rod 1, the transformation matrix $\boldsymbol{T}_{\boldsymbol{O}}^{\boldsymbol{o}_{1}}$ from the local coordinate system $o_{1}-x_{1} y_{1} z_{1}$ to the global coordinate system $O$ $X Y Z$ is

$$
\boldsymbol{T}_{\boldsymbol{o}}^{\boldsymbol{o}_{\mathbf{1}}}=\left[\begin{array}{cccc}
\cos \theta_{a} & 0 & -\sin \theta_{a} & l_{m} \cos \gamma \\
0 & 1 & 0 & l_{4} \\
\sin \theta_{a} & 0 & \cos \theta_{a} & l_{m} \sin \gamma \\
0 & 0 & 0 & 1
\end{array}\right]
$$

the coordinate of $S_{2}$ in $O-X Y Z$ is

$$
S_{2}^{O}=\left[\begin{array}{lll}
l_{3} \cos \left(\theta_{b}+\frac{\pi}{2}-\varphi\right) & l_{4} \sin \theta_{v} & l_{3} \sin \left(\theta_{b}+\frac{\pi}{2}-\varphi\right)
\end{array}\right]^{T}
$$

the coordinate of $S_{2}$ in $o_{1}-x_{1} y_{1} z_{1}$ is

$$
S_{2}^{o_{1}}=\left[\begin{array}{lll}
l_{2} \sin \theta_{s Z} \cos \theta_{s x} & l_{2} \sin \theta_{s z} \sin \theta_{s x} & l_{1}+l_{2} \cos \theta_{s Z}
\end{array}\right]^{T}
$$

the relationship between $S_{2}^{O}$ and $S_{2}^{O_{1}}$ is as follows

$$
\left[\begin{array}{c}
S_{2}^{O} \\
1
\end{array}\right]=T_{O}^{o_{1}}\left[\begin{array}{c}
S_{2}^{o_{1}} \\
1
\end{array}\right]
$$

According to the motion characteristics of the rod 3, the transformation matrix $\boldsymbol{T}_{\boldsymbol{O}}^{\boldsymbol{o}_{2}}$ from $o_{2}-x_{2} y_{2} z_{2}$ to $O-X Y Z$ is

$$
\boldsymbol{T}_{\boldsymbol{o}}^{\boldsymbol{o}_{2}}=\left[\begin{array}{cccc}
\cos \theta_{b} & 0 & -\sin \theta_{b} & 0 \\
0 & 1 & 0 & l_{4}+h_{1} \\
\sin \theta_{b} & 0 & \cos \theta_{b} & 0 \\
0 & 0 & 0 & 1
\end{array}\right]
$$

the coordinates of $S_{3}$ in $O-X Y Z$ and $o_{2}-x_{2} y_{2} z_{2}$ are $S_{3}^{O}=\left[\begin{array}{lll}l_{4} \cos \theta_{v} & l_{4} \sin \theta_{v} & l_{3}\end{array}\right]^{T}$ and $S_{3}^{O_{2}}=\left[\begin{array}{lll}0 & 0 & l_{3}+h_{2}\end{array}\right]^{T}$ respectively. Similarly, $S_{3}^{O}$ and $S_{3}^{O_{2}}$ have the following relationship: 


$$
\left[\begin{array}{c}
S_{3}^{O} \\
1
\end{array}\right]=T_{O}^{o_{2}}\left[\begin{array}{c}
S_{3}^{o_{2}} \\
1
\end{array}\right]
$$

By combining Eqs. (12) (15), we can get:

$$
1-\frac{l_{3}^{2} \sin ^{2} \theta_{b}}{l_{4}{ }^{2} \cos ^{2} \theta_{b}}-2 \sqrt{1-\frac{l_{3}^{2} \sin ^{2} \theta_{b}}{l_{4}^{2} \cos ^{2} \theta_{b}}}=k_{1} \sin \theta_{b}+k_{2} \cos \theta_{b}+k_{3}
$$

where $\quad k_{1}=\frac{-2 l_{3} l_{m} \cos (\gamma+\varphi)+2 l_{1} l_{3} \sin \left(\theta_{a}+\varphi\right)}{l_{4}{ }^{2}}, \quad k_{2}=\frac{2 l_{3} l_{m} \sin (\gamma+\varphi)+2 l_{1} l_{3} \cos \left(\theta_{a}+\varphi\right)}{l_{4}{ }^{2}}$ and $k_{3}=\frac{l_{2}{ }^{2}+2 l_{1} l_{m} \sin \left(\theta_{a}-\gamma\right)-l_{1}{ }^{2}-l_{3}{ }^{2}-l_{4}{ }^{2}-l_{m}{ }^{2}}{l_{4}{ }^{2}}$. Simplify Eq. (16) and let:

$$
f\left(\theta_{b}\right)=\left[1-\left(\frac{l_{3} \sin \theta_{b}}{l_{4} \cos \theta_{b}}\right)^{2}-k_{1} \sin \theta_{b}-k_{2} \cos \theta_{b}-k_{3}\right]^{2}+4\left(\frac{l_{3} \sin \theta_{b}}{l_{4} \cos \theta_{b}}\right)^{2}-4
$$

Eq. (17) is solved by dichotomy method to get $\theta_{b}$, and then the vane rotation angle $\theta_{v}$ is obtained from the following equation:

$$
\theta_{v}=\arccos \left(-\frac{l_{3} \sin \theta_{b}}{l_{4} \cos \theta_{b}}\right)
$$

The detailed derivation process can be seen in Appendix 1.

The rotation velocity $\omega_{i}$, rotation acceleration $\alpha_{i}$ of body $i$ are obtained by numerical method, that is, $\omega_{i}=\frac{d \theta_{i}}{d t}, \alpha_{i}=\frac{d \omega_{i}}{d t}$.

\subsection{Spatial dynamics model of the VSV mechanism}

According to Newton-Euler equation, the forces acting on the components of VSV are described, and the spatial moments can be derived from the following formula:

$$
\begin{gathered}
M_{O}(\vec{F})=\vec{r} \times \vec{F}=\left|\begin{array}{ccc}
\vec{\imath} & \vec{\jmath} & \vec{k} \\
x & y & z \\
F_{x} & F_{y} & F_{z}
\end{array}\right|=\left(y F_{z}-z F_{y}\right) \vec{\imath}+\left(z F_{x}-x F_{z}\right) \vec{\jmath}+\left(x F_{y}-y F_{x}\right) \vec{k} \\
\\
\qquad \begin{array}{l}
M_{O x}(\vec{F})=y F_{z}-z F_{y} \\
M_{O y}(\vec{F})=z F_{y}-x F_{z} \\
M_{O z}(\vec{F})=x F_{y}-y F_{x}
\end{array}
\end{gathered}
$$

where $x, y$ and $z$ are the distances of the force action point relative to the center of rotation.

As shown in Figure 3, the rod 1 rotates under the action of the driving moment $M_{d}$, and is subjected to the constraint forces $F_{a x}, F_{a y}, F_{a z}$ and constraint moments $M_{a x}, M_{a z}$ at the rotary joint $A$, and the constraint forces $F_{21 x}, F_{21 y}, F_{21 z}$ from the rod 2 at the spherical joint $S_{1}$. At the center of mass $O_{1}$, it is subjected to inertial forces 
$F_{1 x i}, F_{1 z i}$, gravity $G_{1}$ and inertial moment $M_{1 y i}$. The relationships between the forces are as follows:

$$
\begin{gathered}
F_{a x}+F_{21 x}+F_{1 x i}=0 \\
F_{a y}+F_{21 y}=0 \\
F_{a z}+F_{21 z}+F_{1 z i}-G_{1}=0
\end{gathered}
$$

In the global coordinate system, the coordinates of $A$ and $S_{l}$ relative to the center of mass $O_{1}$ are $\left(X_{a}^{O_{1}}, Y_{a}^{O_{1}}, Z_{a}^{O_{1}}\right)$ and $\left(X_{s_{1}}^{O_{1}}, Y_{s_{1}}^{O_{1}}, Z_{s_{1}}^{O_{1}}\right)$ respectively, then the moment acting on $O_{1}$ can be expressed as:

$$
\begin{gathered}
-Z_{a}^{O_{1}} F_{a y}-Z_{s_{1}}^{O_{1}} F_{21 y}+M_{a x}=0 \\
X_{a}^{O_{1}} F_{a z}-Z_{a}^{O_{1}} F_{a x}+X_{s_{1}}^{O_{1}} F_{21 z}-Z_{s_{1}}^{O_{1}} F_{21 x}+M_{1 y i}+M_{d}=0
\end{gathered}
$$

Fig. 3 Schematic of the forces on the rod 1

The rod 2 rotates and moves in spatial dimension, as shown in Figure 4, and is restrained by $-F_{21 x},-F_{21 y},-F_{21 z}$ from the rod 1 and $F_{32 x}, F_{32 y}, F_{32 z}$ from the rod 3, respectively. At the center of mass $\mathrm{O}_{2}$, the rod 2 is subjected to inertial forces $F_{2 x i}, F_{2 y i}, F_{2 z i}$, gravity $G_{2}$ and inertial moments $M_{2 x i}, M_{2 y i}, M_{2 z i}$. The relationships between the forces are as follows:

$$
F_{32 x}-F_{21 x}+F_{2 x i}=0
$$




$$
\begin{gathered}
F_{32 y}-F_{21 y}+F_{2 y i}=0 \\
F_{32 z}-F_{21 z}+F_{2 z i}-G_{2}=0
\end{gathered}
$$

In the global coordinate system, the coordinates of $S_{1}$ and $S_{2}$ relative to the center of mass $O_{2}$ are $\left(X_{s_{1}}^{O_{2}}, Y_{s_{1}}^{O_{2}}, Z_{s_{1}}^{O_{2}}\right)$ and $\left(X_{s_{2}}^{O_{2}}, Y_{s_{2}}^{O_{2}}, Z_{s_{2}}^{O_{2}}\right)$ respectively, then the moment acting on $\mathrm{O}_{2}$ can be expressed as:

$$
\begin{aligned}
& Y_{s_{2}}^{O_{2}} F_{32 z}-Z_{s_{2}}^{O_{2}} F_{32 y}-Y_{s_{1}}^{O_{2}} F_{21 z}+Z_{s_{1}}^{O_{2}} F_{21 y}+M_{2 x i}=0 \\
& X_{s_{2}}^{O_{2}} F_{32 z}-Z_{s_{2}}^{O_{2}} F_{32 x}-X_{s_{1}}^{O_{2}} F_{21 z}+Z_{s_{1}}^{O_{2}} F_{21 x}+M_{2 y i}=0 \\
& X_{s_{2}}^{O_{2}} F_{32 y}-Y_{s_{2}}^{O_{2}} F_{32 x}-X_{s_{1}}^{O_{2}} F_{21 y}+Y_{s_{1}}^{O_{2}} F_{21 x}+M_{2 z i}=0
\end{aligned}
$$

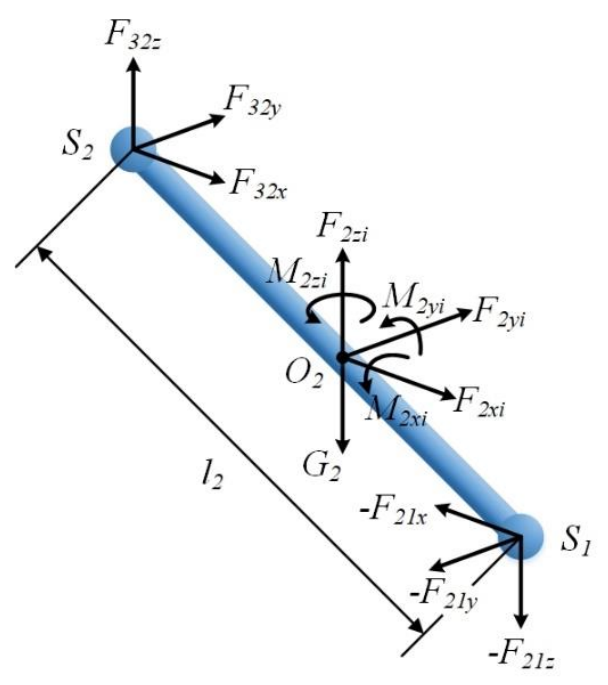

Fig.4 Schematic of the forces on the rod 2

As shown in Figure 5, due to the rotation of the rod 3, the joint ball is subject to the constraint forces $F_{s 3 x}, F_{s 3 y}, F_{s 3 z}$ and the constraint moment $M_{s 3 x}, M_{s 3 y}$ exerted by the pin. It is worth noting that due to the arbitrary rotation of the rod 4 at the spherical joint $S_{3}$ and the mobility of the joint ball along the pin, the rod 4 is not affected by the constraint moment and the constraint force along the $Z$-axis. Therefore, there are only constraint forces $F_{43 x}\left(=F_{s 3 x}\right)$ and $F_{43 y}\left(=F_{s 3 y}\right)$ between the rod 3 and the rod 4. In addition, the rod 2 applies constraint forces $-F_{32 x},-F_{32 y}$ and $-F_{32 z}$ to the rod 3 , and the rod 3 is subjected to inertial forces $F_{3 x i}, F_{3 y i}, F_{3 z i}$, gravity $G_{3}$ and inertial moment $M_{3 y i}$ at the center of mass $O_{3}$. The relationships between the forces are as follows: 


$$
\begin{gathered}
F_{43 x}-F_{32 x}+F_{b x}+F_{3 x i}=0 \\
F_{43 y}-F_{32 y}+F_{3 y i}=0 \\
F_{b z}-F_{32 z}+F_{3 z i}-G_{3}=0
\end{gathered}
$$

In the global coordinate system, the coordinates of $\mathrm{S}_{2}, \mathrm{~S}_{3}$ and the cylindrical pair $\mathrm{B}$ relative to the center of mass $O_{3}$ are $\left(X_{s_{2}}^{O_{3}}, Y_{s_{2}}^{O_{3}}, Z_{s_{2}}^{O_{3}}\right),\left(X_{s_{3}}^{O_{3}}, Y_{s_{3}}^{O_{3}}, Z_{s_{3}}^{O_{3}}\right)$ and $\left(X_{b}^{O_{3}}, Y_{b}^{O_{3}}, Z_{b}^{O_{3}}\right)$ respectively, then the moment acting on $O_{3}$ can be expressed as:

$$
\begin{gathered}
Z_{s_{2}}^{O_{3}} F_{32 y}-Z_{s_{3}}^{O_{3}} F_{43 y}+M_{b x}=0 \\
Z_{s_{2}}^{O_{3}} F_{32 x}-X_{s_{2}}^{O_{3}} F_{32 z}-Z_{b}^{O_{3}} F_{b x}+X_{b}^{O_{3}} F_{b z}-Z_{s_{3}}^{O_{3}} F_{43 x}+M_{3 y i}=0 \\
X_{s_{3}}^{O_{3}} F_{43 y}-X_{s_{2}}^{O_{3}} F_{32 y}+M_{b z}=0
\end{gathered}
$$

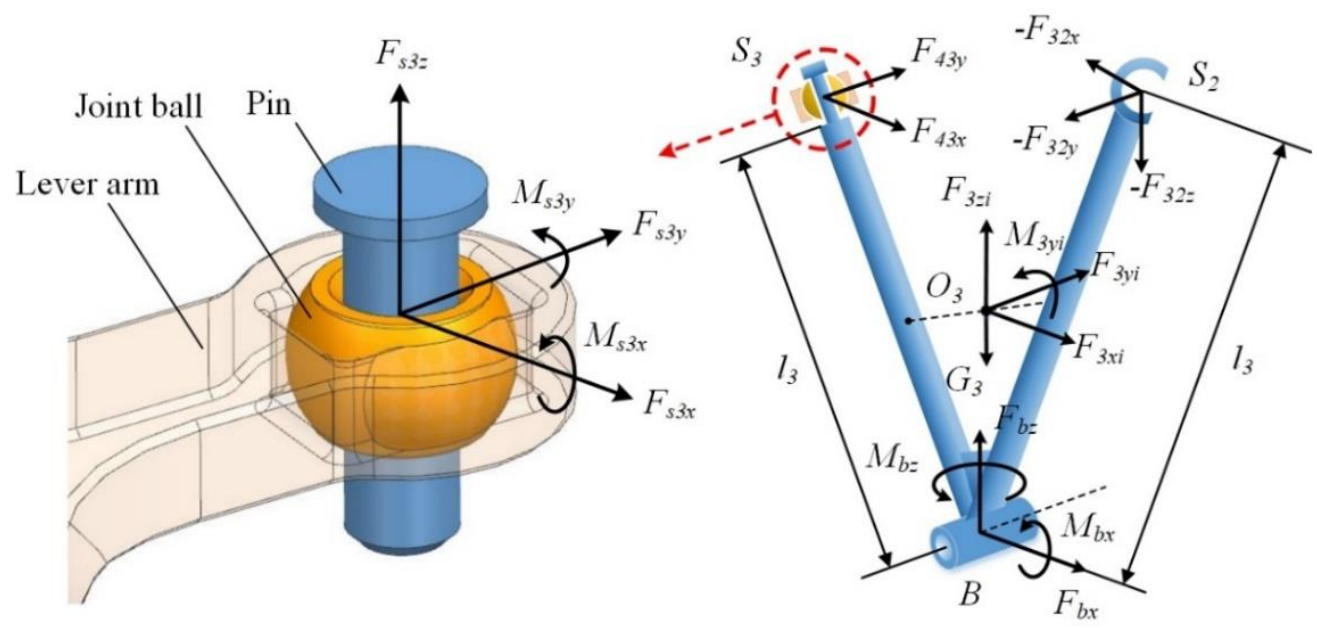

Fig.5 Schematic of the forces on the rod 3

As shown in Figure 6, the lever arm (rod 4) and the vane are fixedly connected, and the rod 4 is far smaller than the vane in size and mass, so the overall center of mass is concentrated on the vane center. The aerodynamic drag on the vane can be simplified as the forces $F_{\text {airx }}, F_{\text {airy }}$ and $M_{\text {air }}$ acting on the mass center $O_{v}$. Due to the clearance at the rotary joint $\mathrm{E}$, the trunnion is subjected to the contact forces $F_{c x}, F_{c y}$ and $F_{c z}$ exerted by the bushing, where $F_{c z}$ is always in balance with the gravity $G_{v}$. In addition, the variations of the contact areas cause the contact force to generate moments $M_{j c x}, M_{j c y}, M_{t c x}$ and $M_{t c y}$ on the trunnion center $O_{j}$, and the rotation of 
the trunnion in the bushing to generate friction moments $M_{j f}$ and $M_{t f}$. The inertia forces $F_{v x i}, F_{v y i}$ and inertia moment $M_{v x i}, M_{v y i}$ and $M_{v z i}$ are applied to the center of mass $O_{v}$, and the constraint force $-F_{43 x},-F_{43 y}$ is applied to the rod 4 at the spherical joint $S_{3}$. The relationships between the forces are as follows:

$$
\begin{gathered}
F_{c x}-F_{43 x}+F_{\text {airx }}+F_{v x i}=0 \\
F_{c y}-F_{43 y}+F_{\text {airy }}+F_{v y i}=0 \\
F_{c z}-G_{4}=0
\end{gathered}
$$

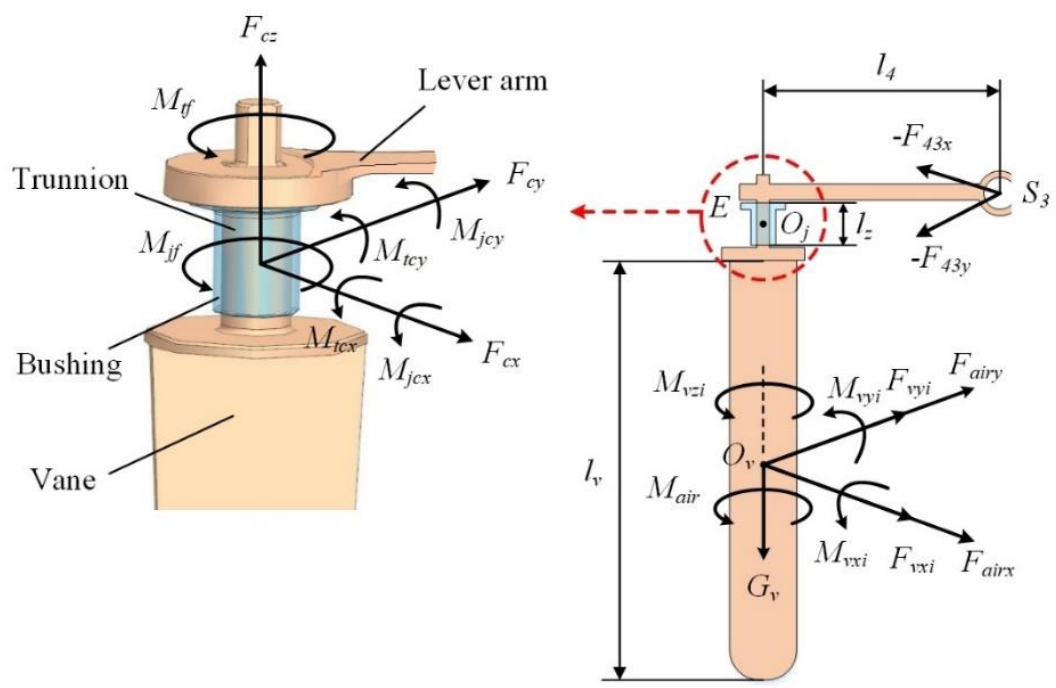

Fig.6 Schematic of the forces on the lever arm and the vane

In the global coordinate system, the coordinates of $S_{3}$ relative to the center of the trunnion $O_{j}$ are $\left(X_{s_{3}}^{O_{j}}, Y_{s_{3}}^{O_{j}}, Z_{s_{3}}^{O_{j}}\right)$, then the moment acting on $O_{j}$ can be expressed as:

$$
\begin{gathered}
M_{j c x}+M_{t c x}+Z_{s_{3}}^{O_{j}} F_{43 y}+\frac{l_{v}}{2} F_{a i r y}+\frac{l_{v}}{2} F_{v y i}+M_{v x i}=0 \\
M_{j c y}+M_{t c y}+Z_{s_{3}}^{O_{j}} F_{43 x}+\frac{l_{v}}{2} F_{a i r x}+\frac{l_{v}}{2} F_{v x i}+M_{v y i}=0 \\
Y_{s_{3}}^{O_{j}} F_{43 x}-X_{s_{3}}^{O_{j}} F_{43 y}+M_{j f}+M_{t f}+M_{a i r}+M_{v z i}=0
\end{gathered}
$$

In the above formulas, the inertia forces and moments are expressed as $F_{\lambda i}=$ $-m_{\lambda} a_{\lambda}, M_{\lambda i}=-J_{\lambda} \alpha_{\lambda}$, where $m_{\lambda}, a_{\lambda}, J_{\lambda}$ and $\alpha_{\lambda}$ are the mass, acceleration, moment of inertia and angular acceleration of component $\lambda$ respectively. The detailed expressions of the inertia forces, moments and the coordinates of the joints are shown in the Appendix 2 
The contact forces $F_{c x}, F_{c y}$ and the contact moment $M_{j c x}, M_{j c y}$ are obtained by integrating the contact pressure $p_{c}$ and the moment of $p_{c}$ to the center of the trunnion $O_{j}$ on the contact area $A_{j}$ respectively. The supporting force $F_{c z}$ of the bushing to the lever arm also generates the moment to the point $O_{j}$, namely, $M_{t c x}$ and $M_{t c y}$. The formulas for calculation are as follows:

$$
\left\{\begin{array}{c}
F_{c x}=\iint_{A_{j}} p_{c} \cos \theta\left(r_{b} d \theta d z\right) \\
F_{c y}=\iint_{A_{j}} p_{c} \sin \theta\left(r_{b} d \theta d z\right) \\
M_{j c x}=-\iint_{A_{j}} p_{c} \sin \theta\left(r_{b} d \theta d z\right) \cdot z_{j} \\
M_{j c y}=-\iint_{A_{j}} p_{c} \cos \theta\left(r_{b} d \theta d z\right) \cdot z_{j} \\
M_{t c x}=-F_{c z} \sin \left(\arctan \frac{\varphi_{x}}{\varphi_{y}}\right) \cdot r_{t} \\
M_{t c y}=-F_{c z} \cos \left(\arctan \frac{\varphi_{x}}{\varphi_{y}}\right) \cdot r_{t}
\end{array}\right.
$$

The calculation formula of friction moment caused by the trunnion rotation is as follows:

$$
\left\{\begin{array}{c}
M_{j f}=\iint_{A_{j}} \mu_{f} p_{c} \operatorname{sig}\left(\omega_{v}\right)\left(r_{b} d \theta d z\right) \cdot r_{b} \\
M_{t f}=\mu_{f} F_{c z} \operatorname{sig}\left(\omega_{v}\right) \cdot r_{t}
\end{array}\right.
$$

where $r_{b}$ and $l_{z}$ are the radius and the height of bushing, $\theta$ is the circumference coordinate, $r_{t}$ is the radius of the lever arm support surface, $z_{j}$ is the distance between the contact point and $O_{j}$, which is positive along the $Z$-axis and negative on the contrary. $\operatorname{sig}\left(\omega_{v}\right)=\left\{\begin{array}{cc}-1 & \omega_{v}>0 \\ 1 & \omega_{v}<0\end{array}, \mu_{f}\right.$ is the coefficient of friction between the trunnion and the bushing. The detailed description of these symbols can refer to Figure 7.

Figure 7(a) shows the spatial attitude of the trunnion in the bushing at a certain time, and Figure 7(b) is a plane diagram. The deviation of the trunnion along $x$ and $y$ direction is defined as $e_{x}$ and $e_{y}$, and the deflection angle around $x$ and $y$ axis is defined as $\varphi_{x}$ and $\varphi_{y}$. Next, the dynamics equation is established to solve the four independent variables. 


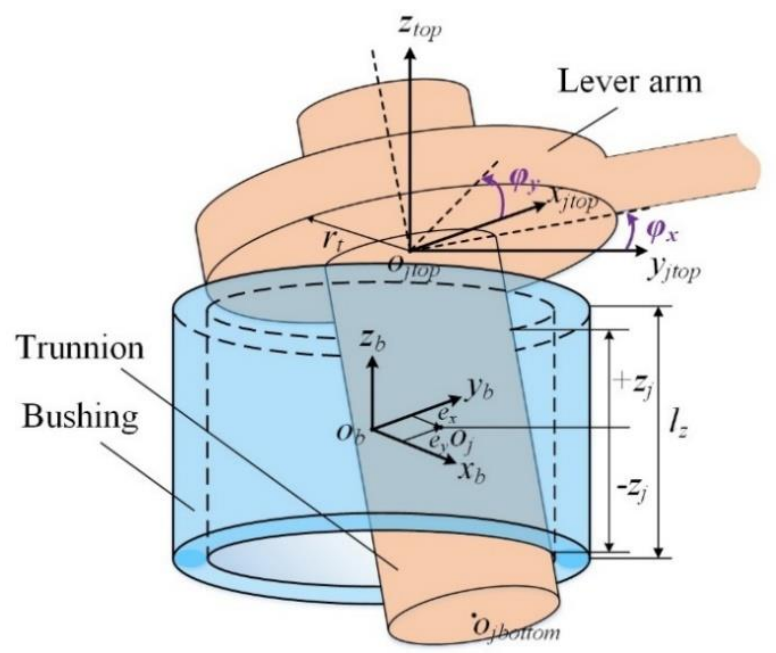

(a)

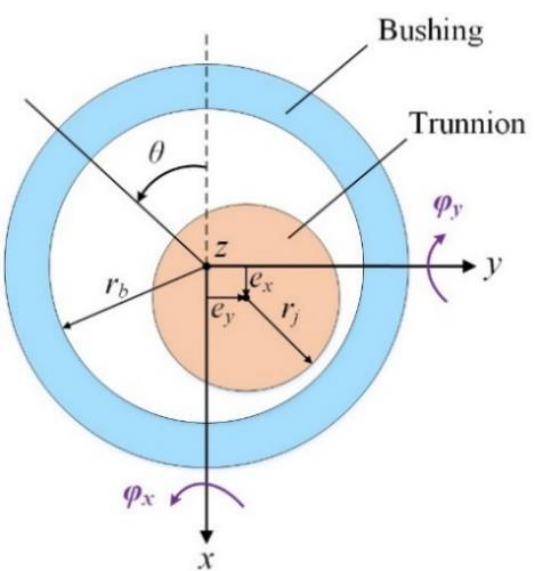

(b)

Fig.7 Schematic diagram of attitude of the trunnion in the bushing

By combining Eqs. (20) (43) and eliminating the constraint forces, a dynamics matrix containing four independent variables is obtained:

$$
\left[\begin{array}{llll}
A_{11} & A_{12} & A_{13} & A_{14} \\
A_{21} & A_{22} & A_{23} & A_{24} \\
A_{31} & A_{32} & A_{33} & A_{34} \\
A_{41} & A_{42} & A_{43} & A_{44}
\end{array}\right]\left[\begin{array}{c}
\ddot{e}_{x} \\
\ddot{e}_{y} \\
\ddot{\varphi}_{x} \\
\ddot{\varphi}_{y}
\end{array}\right]=\left[\begin{array}{l}
B_{11} \\
B_{21} \\
B_{31} \\
B_{41}
\end{array}\right]
$$

the intermediate variables $A_{11} \sim A_{44}$ and $B_{11} \sim B_{41}$ are given in the Appendix 2.

\subsection{Wear model}

In this study, it is assumed that wear occurs only on the surface of the bushing due to the material is soft. According to Archard wear model [31], the wear depth $h_{\text {wear }}$ of the bushing can be expressed as:

$$
h_{\text {wear }}=\frac{C_{w}}{H_{d}} \cdot t_{\text {step }} \cdot \bar{W}_{L}
$$

where $C_{w}$ is the wear coefficient, $H_{d}$ is the hardness of the bushing, and $t_{\text {step }}$ is the relevant step time. Here, the material of bushing is polyimide, the reference value of hardness $H_{d}$ is $65 \mathrm{HV}$, and the wear coefficient is assumed as a constant $C_{w}=$ $1.1 \times 10^{-15} \mathrm{~m}^{2} \mathrm{~N}^{-1}$, the trunnion is made of stainless steel. The wear load $\bar{W}_{L}$ is the average contact pressure of the vane in a rotation period $T$, which can be expressed as: 
$\bar{W}_{L}=\frac{1}{T} \int_{t}^{t+T} p_{c}|u| d t$, and $u$ is the sliding speed of the trunnion, $u=\omega_{v} \cdot r_{j}$.

Therefore, the separation distance $h$ between the trunnion and the bushing can be expressed as:

$$
h=c+\left(e_{x}-z_{j} \tan \varphi_{y}\right) \cos \theta+\left(e_{y}-z_{j} \tan \varphi_{x}\right) \sin \theta+h_{w e a r}
$$

where $c$ is the radial clearance, that is, $c=r_{b}-r_{j}$.

\section{Numerical method}

The tribo-dynamics of the VSV spatial mechanism contains many nonlinear ordinary differential equations (ODEs). To solve these ODEs, the first step is to express the four second-order variables in Eq. (45) as first-order ODEs:

$$
\boldsymbol{y}=\left(e_{x}, e_{y}, \varphi_{x}, \varphi_{y}, \dot{e}_{x}, \dot{e}_{y}, \dot{\varphi}_{x}, \dot{\varphi}_{y}\right)^{T}
$$

and the first derivative of $\boldsymbol{y}$ is

$$
\boldsymbol{y}^{\prime}=\left(\dot{e}_{x}, \dot{e}_{y}, \dot{\varphi}_{x}, \dot{\varphi}_{y}, \ddot{e}_{x}, \ddot{e}_{y}, \ddot{\varphi}_{x}, \ddot{\varphi}_{y}\right)^{T}
$$

According to the initial state of the trunnion center, set the initial value of $y_{0}=$ $y\left(t_{0}\right)=\left(e_{x}^{0}, e_{y}^{0}, \varphi_{x}^{0}, \varphi_{y}^{0}, \dot{e}_{x}^{0}, \dot{e}_{y}^{0}, \dot{\varphi}_{x}^{0}, \dot{\varphi}_{x}^{0}\right)^{T}$, and then the initial value problem in the firstorder ODEs is obtained as follows:

$$
\boldsymbol{y}^{\prime}=f(t, y), \boldsymbol{y}_{0}=\boldsymbol{y}\left(t_{0}\right)
$$

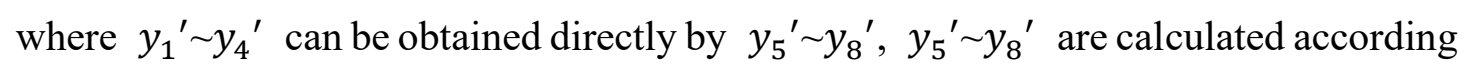
to Eq. (46).

The modified extended backward differentiation formulae (MEBDF) proposed by Cash [32] is adopted to solve Eq. (51), and it has been proved stable and efficient in solving the nonlinear ODEs. For more details on the application of MEBDF can be found in Ref. [33,34]. 


\section{Application and analysis of the RSDC model}

\subsection{RSDC model under dry friction condition}

In this section, the proposed RSDC model is used to simulate a simple physical object: journal bearing under the condition of dry friction and steady loads, in order to show the beneficial effect of the model in dynamics calculation. The motion diagram of the journal in the bearing can be referred to Figure 7 (b). Here, it is assumed that the journal does not rotate, and the center of the journal is subjected to the stable loads $W_{x}=200 N$ in the $X$ direction and $W_{y}=100 N$ in the $Y$ direction respectively, so the journal only deviates $\left(e_{x}, e_{y}\right)$ in the $X$ and $Y$ directions without deflection $\left(\varphi_{x}, \varphi_{y}\right)$. The radial clearance between bearing and journal is set as $20 \mu \mathrm{m}$, and the basic parameters of journal and bearing correspond to the trunnion and bushing in VSV mechanism respectively. It is specially noted that the trunnion and the vane are integrated, and their total mass $M_{v}$ is used as the mass of the journal $M_{\text {ass }}$ for simulation. The values of each parameter are shown in Table 1.

Table 1 Basic parameters of the trunnion and the bushing.

\begin{tabular}{cccc}
\hline Parameter & Value & Parameter & Value \\
\hline$m_{v}$ & $0.253(\mathrm{~kg})$ & $\sigma_{1}$ & $0.392(\mu \mathrm{m})$ \\
$r_{j}$ & $6(\mathrm{~mm})$ & $\sigma_{2}$ & $1.586(\mu \mathrm{m})$ \\
$l_{z}$ & $20(\mathrm{~mm})$ & $E_{1}$ & $203(\mathrm{GPa})$ \\
$\xi$ & $0.038\left(\mu \mathrm{m}^{-2}\right)$ & $E_{2}$ & $7.17(\mathrm{GPa})$ \\
$\beta$ & $8.17(\mu \mathrm{m})$ & $v_{1}$ & 0.27 \\
$H$ & $492(\mathrm{MPa})$ & $v_{2}$ & 0.34 \\
\hline
\end{tabular}

The deviations of the journal and the contact forces provided by the bearing are shown in Figure 8, in which the calculation results of the RSDC contact model and KE model are compared. It can be seen from the Figure 8 (a) that the RSDC model shows strong stability in the calculation process, that is, after the initial contact, the journal gradually stabilizes in a appropriate position, and finally $e_{x}$ and $e_{y}$ remain constant. However, the calculation result of KE model is oscillatory, and with the calculation 
process, the oscillation intensifies. Gradually, the deviation of the journal is larger than the radial clearance, which means that the journal penetrates the bearing. The variation of journal deviations is also reflected in the contact forces, as shown in Figure 8 (b). During the initial contact, the contact forces $F_{c x}$ and $F_{c y}$ increase sharply due to the high-speed impact of journal, but the RSDC model still maintains the stability of the contact forces. In the KE model, only the interference of the surface asperities provides the rigid contact forces, which causes the distance of the journal rebound to be greater than the distance of the initial state, so the interference is more serious when they contact again. Eventually, the journal will penetrate the bearing and produce extremely large contact force. This phenomenon can be attributed to the damping dissipates the kinetic energy of the journal in the contact process, and the steady-state equilibrium can be formed only when the energy decays. In other words, the contact model without damping will satisfy the conservation of kinetic energy or even increase the kinetic energy, so the dynamics of the system is difficult to be stable.

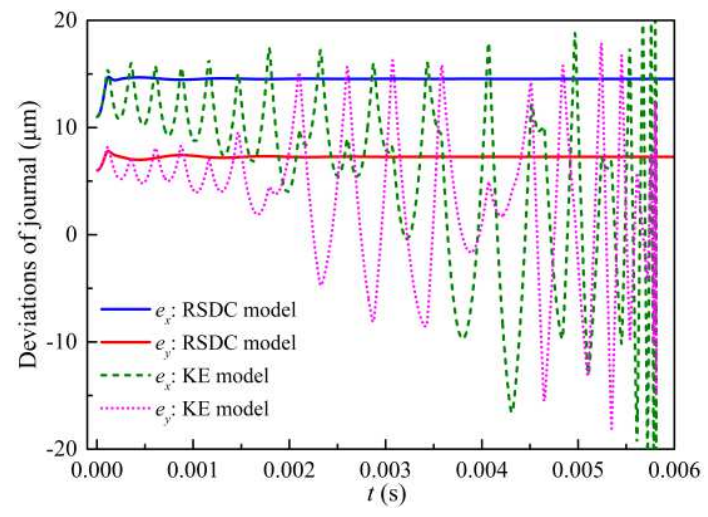

(a)

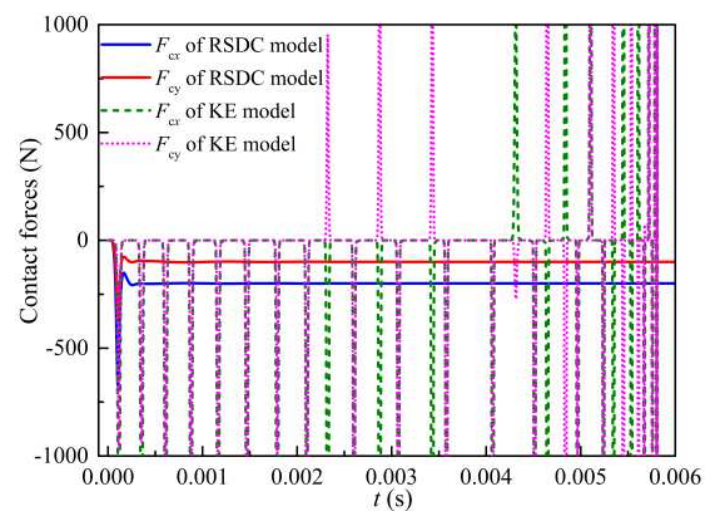

(b)

Fig. 8 Journal bearing with dry friction: (a) deviations of journal, (b) contact forces

\subsection{RSDC model under lubrication condition}

In the actual working condition, there is lubricating oil in the bearing, and the oil film force provided by the lubricating oil and the contact force provided by the bearing play a supporting role together. Not only that, the lubricating medium can also provide effective damping effect. In order to prove the damping effect of lubricating oil, the journal bearing under the action of stable loads is still selected as the simulation object 
in this section, and the load values and the parameters of journal bearing are derived from Section 4.1. The average Reynolds equation is used to calculate the oil film pressure, the expression is as follows:

$$
\frac{1}{r_{j}^{2}} \frac{\partial}{\partial \theta}\left(\phi_{\theta} \frac{h^{3}}{\eta} \frac{\partial p_{o}}{\partial \theta}\right)+\frac{\partial}{\partial z}\left(\phi_{z} \frac{h^{3}}{\eta} \frac{\partial p_{o}}{\partial z}\right)=6 \omega \phi_{c} \frac{\partial h}{\partial \theta}-6 \omega \sigma \frac{\partial \phi_{s}}{\partial \theta}+12 \phi_{c} \frac{\partial h}{\partial t}
$$

where $p_{o}$ is the average lubricant pressure, the meaning of $h$ is the same as that in Eq. (48), and it can also indicate the oil film thickness here. $\eta$ is the viscosity of lubricating oil, $\eta=0.01 \mathrm{~Pa} \cdot \mathrm{s} . \phi_{\theta}$ and $\phi_{z}$ are the pressure flow factors, $\phi_{s}$ is the shear flow factor [35,36], and $\phi_{c}$ is the contact factor [37]. $\omega$ is the rotational speed of the journal, $\omega=0$, so only the squeeze action of the oil film provides the support force.

The Reynolds boundary conditions are applied to solve Eq. (52), which can be expressed as:

$$
\begin{aligned}
p_{o}(\theta, z) & =0, & & z \in\left(0, l_{z}\right) \\
\frac{\partial p_{o}}{\partial \theta} & =0, & & \theta=\theta^{\prime}
\end{aligned}
$$

where $\theta^{\prime}$ is the circumferential position of the oil film rupture.

The oil film forces $F_{o x}$ ( $X$ direction) and $F_{o y}$ ( $Y$ direction) can be obtained by integrating the oil film pressure on the pressure distribution area. The integral formulas are as follows:

$$
\left\{\begin{array}{l}
F_{o x}=\iint_{A_{j}} p_{o} \cos \theta\left(r_{j} d \theta d z\right) \\
F_{o y}=\iint_{A_{j}} p_{o} \sin \theta\left(r_{j} d \theta d z\right)
\end{array}\right.
$$

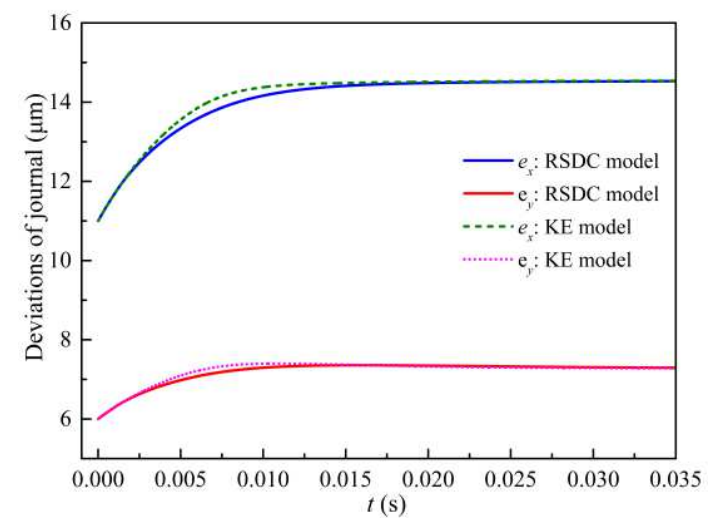

(a)

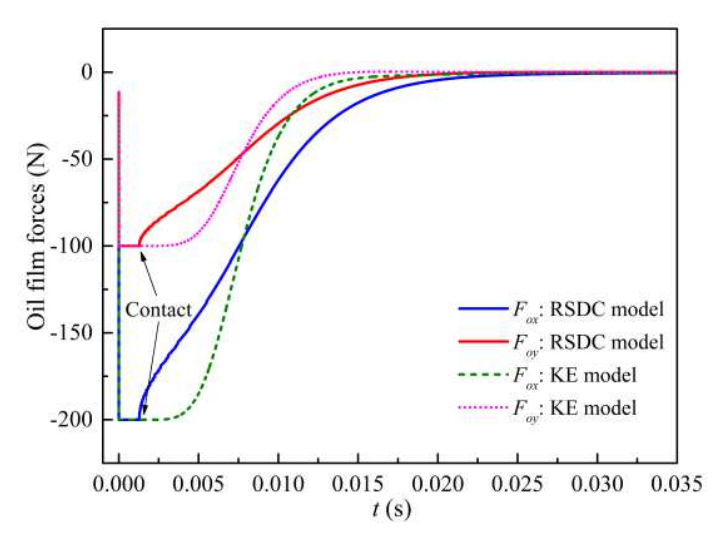

(b) 


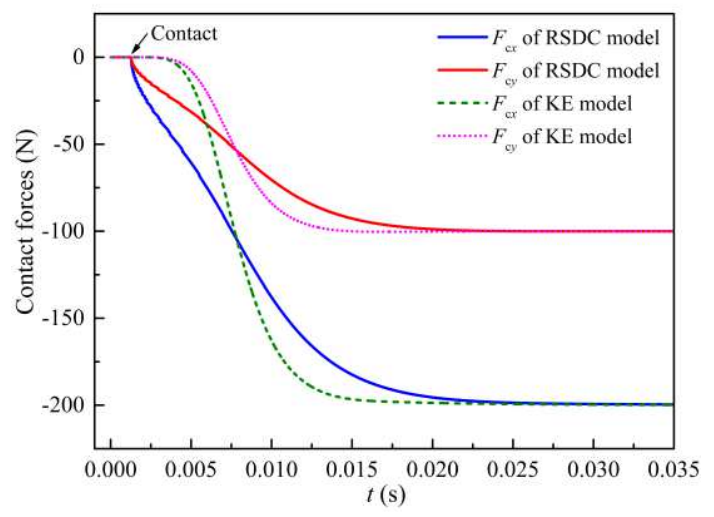

(c)

Fig. 9 Journal bearing with oil lubrication: (a) deviations of journal (b) oil film forces, (b) contact forces

Under the condition of lubricating oil, the deviations and forces of the journal in the bearing are shown in Figure 9. No matter the RSDC model or the KE model is used to calculate the contact forces, a stable calculation process is realized under the action of lubricant. In Figure 9 (a), the variations of journal deviations obtained by the two contact models are smooth curves, and the trajectories are almost coincident. This shows that the contact models have little influence on the journal motion in the presence of lubricating medium. Under the action of external loads, the journal compresses the oil film, so the lubricating oil rapidly produces the support forces. When the journal starts to contact with the bearing, the contact forces are generated, and the oil film forces are reduced accordingly, as shown in Figure 9 (b) and (c). It can be seen from Figure 9 (b) that when the RSDC model is used to calculate the contact forces, the oil film forces decrease rapidly after contact, while when the KE model is used, the oil film forces decrease slowly. It is also shown in Figure 9 (c) that in the initial stage of contact, the contact forces produced by the RSDC model are significant, while those produced by the KE model are very small. This is because the contact forces in the RSDC model is not only from the deformation of the asperities, but also from the impact velocity. However, the contact forces in the KE model only depend on the former, so the contact forces are not obvious when the interferences are just generated. Even so, the calculation process of the two models is stable under the action of lubricating oil, and 
the final contact forces are balanced with the external loads. From these results, it can be seen that the damping effect of lubricating oil is more significant than that of friction pairs materials, which is due to its viscosity. Therefore, the dynamics simulation process of clearance pair with lubricating medium can be stable even if rigid contact model is used. However, the joints of the VSV mechanism are dry friction condition, so the calculation of contact forces should adopt the model with damping.

\section{Tribo-dynamics analysis of the VSV mechanism}

\subsection{Rotation angle and velocity of the vane}

The material parameters of the trunnion and the bushing can be seen in Table 1, and the structural parameters of the VSV mechanism are shown in Table 2.

Table 2 Basic parameters for the VSV mechanism

\begin{tabular}{cccc}
\hline Parameter & Value & Parameter & Value \\
\hline$m_{1}$ & $0.82(\mathrm{~kg})$ & $l_{m}$ & $500(\mathrm{~mm})$ \\
$m_{2}$ & $0.1232(\mathrm{~kg})$ & $r_{t}$ & $12(\mathrm{~mm})$ \\
$m_{3}$ & $0.6744(\mathrm{~kg})$ & $J_{1}$ & $6.22 \times 10^{-4}\left(\mathrm{~kg} \cdot \mathrm{m}^{2}\right)$ \\
$l_{1}$ & $47.7(\mathrm{~mm})$ & $J_{2}$ & $8.33 \times 10^{-4}\left(\mathrm{~kg} \cdot \mathrm{m}^{2}\right)$ \\
$l_{2}$ & $142.4(\mathrm{~mm})$ & $J_{3}$ & $7.20 \times 10^{-2}\left(\mathrm{~kg} \cdot \mathrm{m}^{2}\right)$ \\
$l_{3}$ & $400(\mathrm{~mm})$ & $J_{v}$ & $2.16 \times 10^{-3}\left(\mathrm{~kg} \cdot \mathrm{m}^{2}\right)$ \\
$l_{4}$ & $50(\mathrm{~mm})$ & $\varphi$ & $55\left({ }^{\circ}\right)$ \\
$l_{v}$ & $160(\mathrm{~mm})$ & $\gamma$ & $20\left(^{\circ}\right)$ \\
\hline
\end{tabular}

In this study, given the input rotation speed $\omega_{a}=20 \sin 1 t\left({ }^{\circ} \cdot s^{-1}\right)$ and the starting angle $\theta_{a}^{0}=-16^{\circ}$ of the crank to simulate the tribo-dynamics of the VSV mechanism, as shown in Figure 10. The rotation angle of the vane $\theta_{v}$ in a complete cycle is calculated according to Eq. (18), and the rotation speed $\omega_{v}$ is obtained by differentiating the time with the rotation angle. The results are shown in Figure 11, it can be seen that the rotation range of the vane is $60^{\circ}$ to $120^{\circ}$. In addition, the direction 
of aerodynamic drag will change when the vane reverses. The specific value of aerodynamic drag is shown in Table 3.

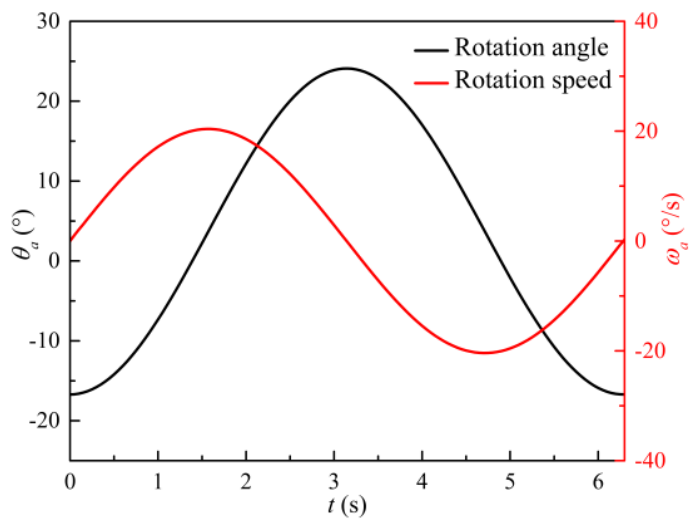

Fig. 10 Driving angle and speed of the crank

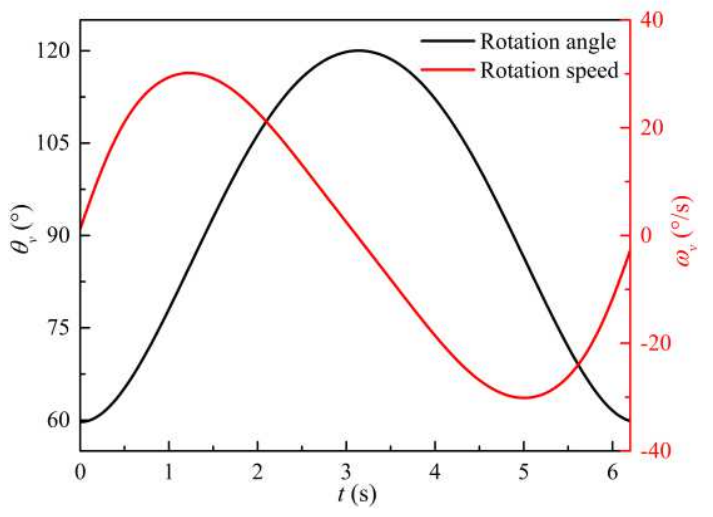

Fig. 11 Rotation angle and speed of the vane

Table 3 Aerodynamic drag acting on a single vane

\begin{tabular}{cccccc}
\hline & \multicolumn{2}{c}{$F_{\text {air }}(\mathrm{N})$} & $F_{\text {airy }}(\mathrm{N})$ & \multicolumn{2}{c}{$M_{\text {air }}(\mathrm{Nm})$} \\
\hline Vane State & $60^{\circ}<\theta_{v}<90^{\circ}$ & $90^{\circ}<\theta_{v}<120^{\circ}$ & & $\omega_{v}>0$ & $\omega_{v}<0$ \\
Value & -0.4 & 0.4 & 1.2 & -0.84 & 0.84 \\
\hline
\end{tabular}

\subsection{Dynamics analysis of the trunnion}

Based on the tribo-dynamics and the RSDC model established in this paper, the contact forces between the trunnion and the bushing with a clearance of $20 \mu \mathrm{m}$ are calculated. As shown in Figure 12, the contact forces $F_{c x}$ and $F_{c y}$ are periodic during the two cycles of adjustment, where $F_{c x}$ presents a positive and negative transformation with the direction of vane rotation, but $F_{c y}$ is almost always negative 
due to the effect of the main aerodynamic resistance $F_{\text {airy }}$. In addition, $F_{c x}$ is always larger than $F_{c y}$, which indicates that the interference distance of the asperities is more serious in $X$ direction. The contact forces include rigid forces $\left(F_{k x}, F_{k y}\right)$, damping forces $\left(F_{d x}, F_{d y}\right)$, and the rigid forces are dominant. When the rotation direction of the vane is changed, the action direction of the driving forces and the resistance moment $M_{\text {air }}$ will be reversed, and the interference velocity and distance will also vary accordingly, which cause the fluctuation of rigid forces and damping forces. This phenomenon can be seen in the enlarged pictures in Figure 12. However, the rigid forces and damping forces compensate each other to make the contact forces stable, so that the system still maintains the balance of the forces at the moment of intense contact.

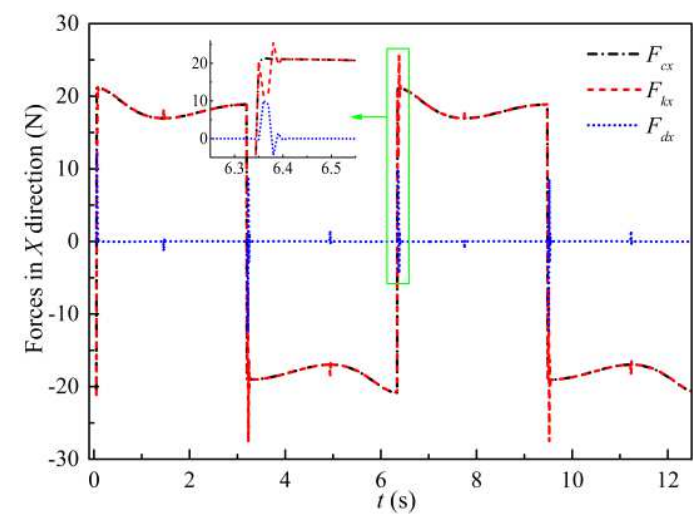

(a)

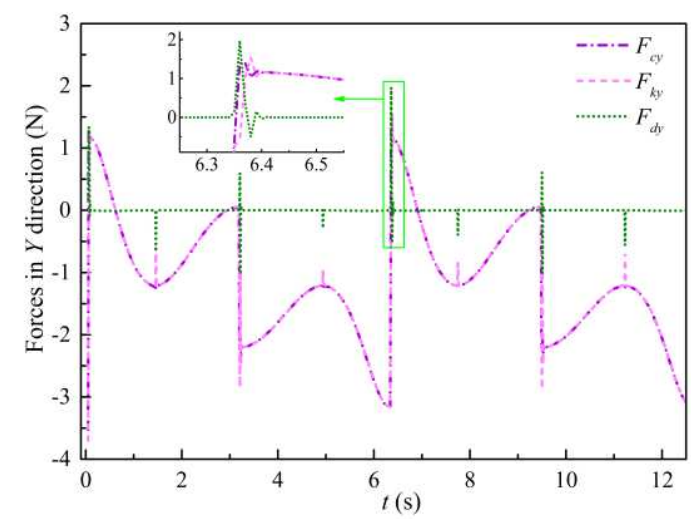

(b)

Fig. 12 Contact, rigid and damping forces acting on the trunnion: (a) $X$ direction, (b) $Y$ direction

In the cyclic operation, the movement of the trunnion in the bushing is shown in Figure 13. Obviously, the deviations of the top center $O_{j t o p}$, the middle center $O_{j}$, and the bottom center $O_{j b o t t o m}$ of the trunnion in the $X$ direction are more obvious than that in the $Y$ direction, that is, the contact areas are concentrated in the $X$ direction, which also proves the performance of the contact forces in Figure 12. It can be seen from the continuous trajectories of the three centers that a tilt angle always exists in the whole movement process, which is due to the deflection of the trunnion around the $X$ axis caused by the main aerodynamic drag $F_{\text {airy }}$. In brief, the driving forces of the unison ring acting on the lever arm make the trunnion deviate obviously in the $X$ direction, and $F_{\text {airy }}$ makes the trunnion deflect around the $X$-axis.

The deviation velocities of the three centers $O_{j t o p}, O_{j}$, and $O_{j b o t t o m}$ in the $X$ and 
$Y$ directions are shown in Figure 14, in which the velocities of the trunnion are small in the continuous adjustment process, but when the vane changes the direction of rotation, the velocities will increase suddenly, corresponding to the variations of displacement in Figure 13. In Figure 14 (a), the velocities of the three centers are approximate in value and consistent in direction, which is due to the adjustment function of the mechanism. However, there are obvious differences in the velocity of the three centers in the $Y$ direction, and the velocity of $O_{j t o p}$ and $O_{j b o t t o m}$ is opposite, as shown in Figure 14 (b), which is due to the moment generated by the main aerodynamic drag $F_{\text {airy }}$ on the center $O_{j}$, resulting in the deflection of the trunnion around the $X$-axis.

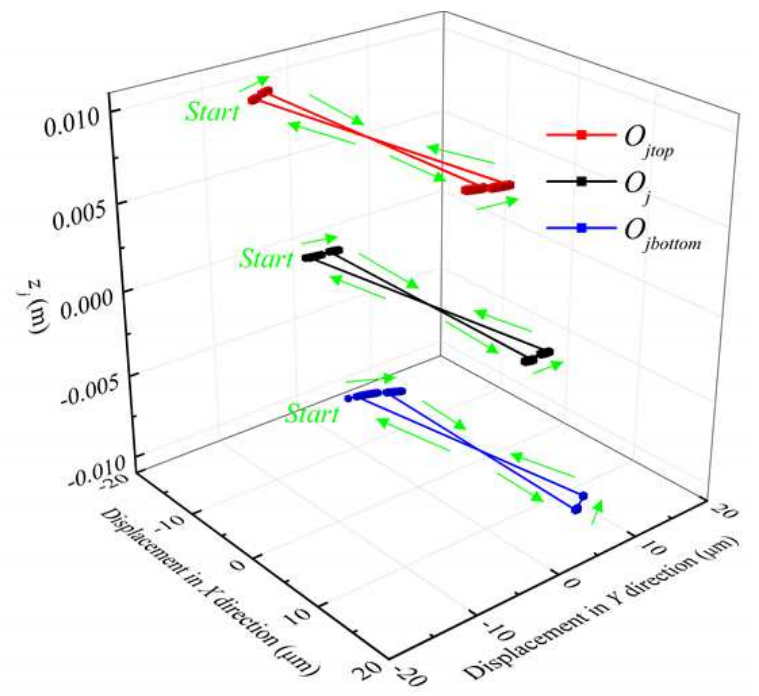

Fig. 13 Trajectory of the trunnion

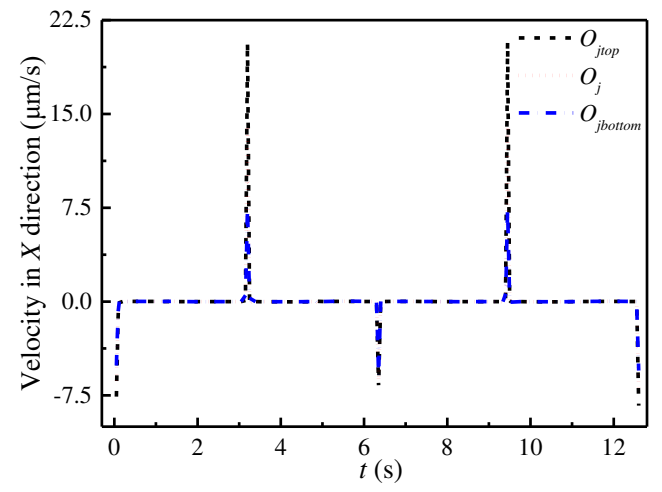

(a)

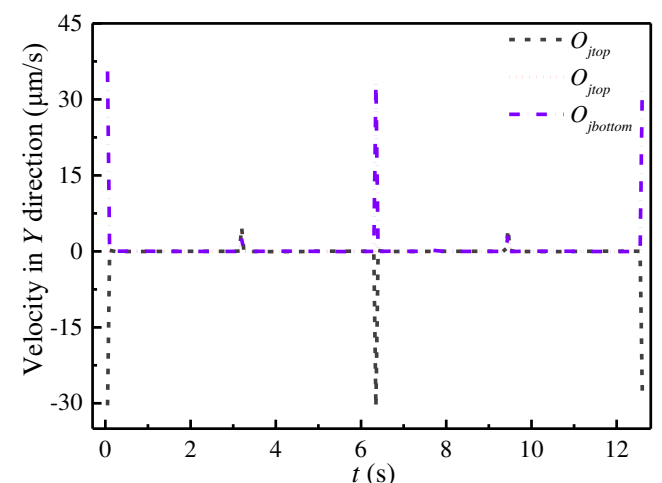

(b)

Fig. 14 Velocity of the three centers of the trunnion in (a) $\mathrm{X}$ direction and (b) $\mathrm{Y}$ direction

Figure 15 shows the contact pressure distribution between the trunnion and the bushing at 6 locations $\left(60^{\circ}, 90^{\circ}, 120^{\circ}\right.$ in forward rotation and $120^{\circ}, 90^{\circ}, 60^{\circ}$ in reverse 
rotation) during the complete cycle of the vane, from which it can be seen that the contact areas are concentrated at the edge. In the forward rotation state, the cloud images of contact pressure fields with vane rotation angles of $60^{\circ}, 90^{\circ}$ and $120^{\circ}$ are shown in figures $(\mathrm{a}) \sim(\mathrm{c})$, and the contact areas occur at $300^{\circ}$ to $45^{\circ}$ of the circumferences. When the vane angles are $120^{\circ}, 90^{\circ}$ and $60^{\circ}$ in reverse rotation, the cloud images of the contact pressure fields are (d) (f), and the contact areas are at 135 $\sim 225^{\circ}$ of the circumference. There are also differences in the contact pressure values and axial distribution in figures (a) (f), which are caused by the reversal of aerodynamic drag and the instantaneous change of driving forces.

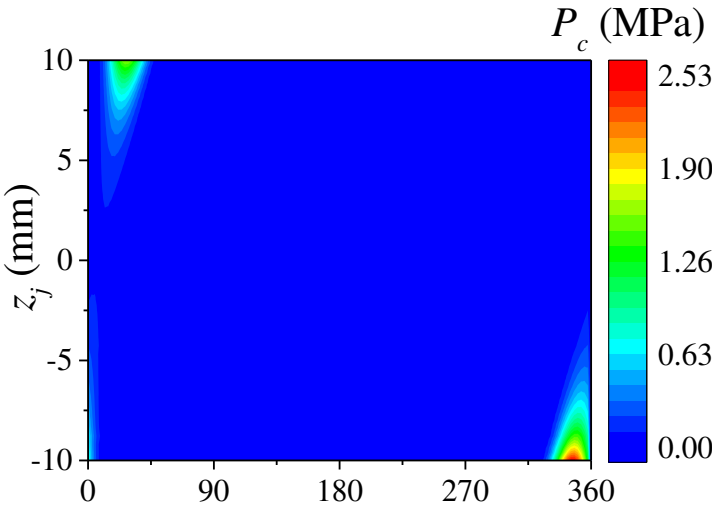

(a)

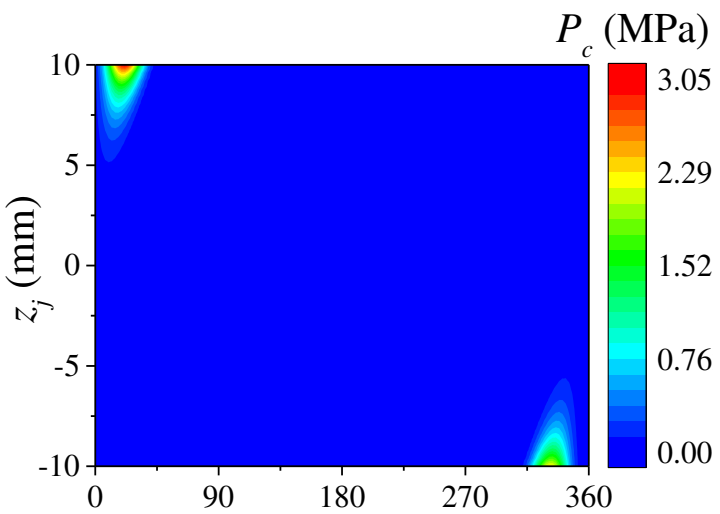

(c)

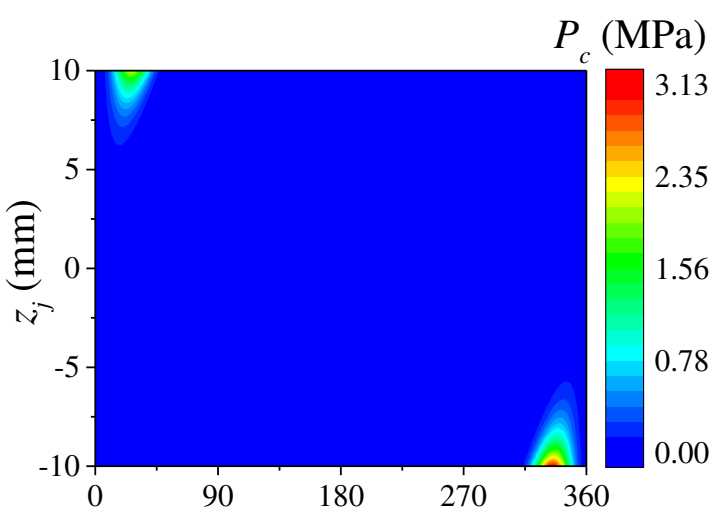

(b)

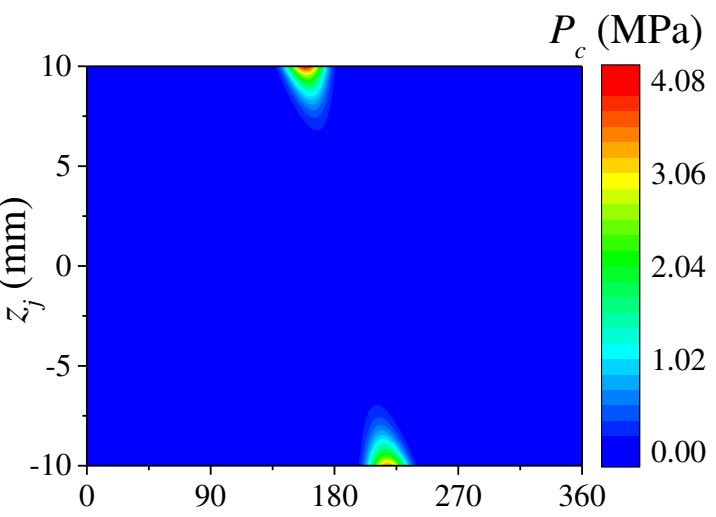

(d) 


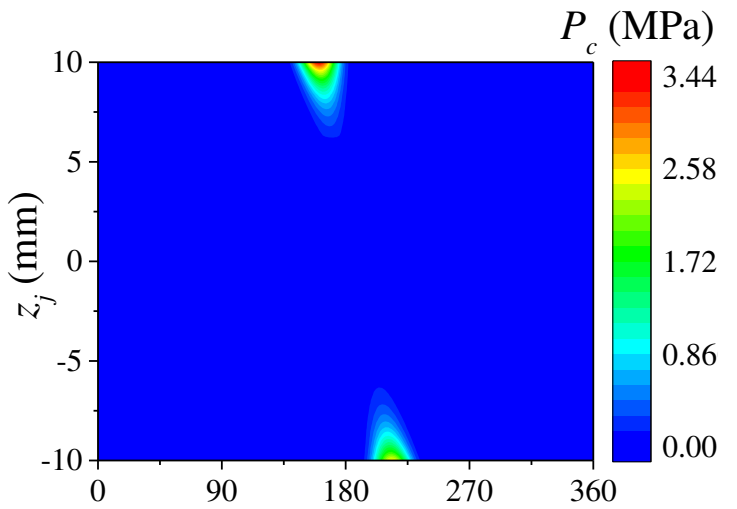

(e)

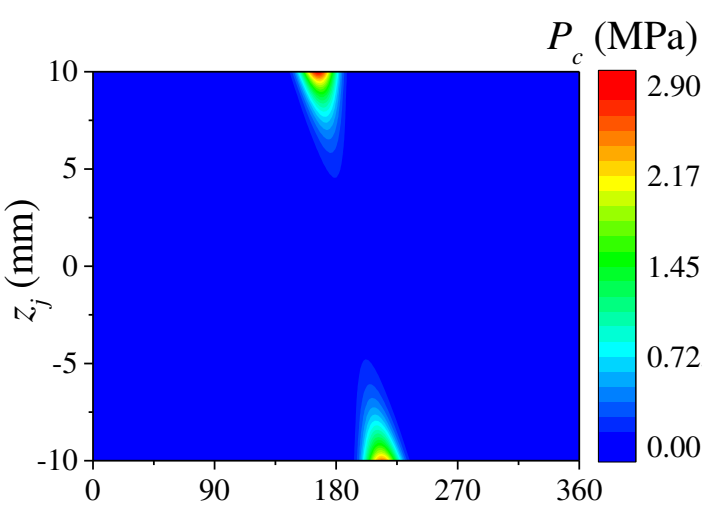

(f)

Circumferential position $\left(^{\circ}\right)$

Fig.15 The contact pressure distribution of the vane at different positions: (a) $60^{\circ}$, (b) $90^{\circ}$, (c) $120^{\circ}$ in forward rotation, (d) $120^{\circ}$, (e) $90^{\circ}$, (f) $60^{\circ}$ in reverse rotation

\subsection{Effect of clearance size}

The effect of clearance size on the VSV mechanism is studied in the range of 20 $\mu \mathrm{m}$ to $40 \mu \mathrm{m}$. In one cycle, the rotation angle and rotation velocity of the vane with different clearance sizes are shown in Figure 16. Figure 16 (a) shows the variation of rotation angle with time, from which it can be seen that the error of rotation angle caused by the clearance of $20 \mu \mathrm{m}$ is about $0.02^{\circ}$ compared with the ideal pair. Figure 16 (b) shows that the difference of rotation velocity between the clearance of $20 \mu \mathrm{m}$ and the ideal pair is about $2.5 \times 10^{-4} \% \mathrm{~s}$. According to the error of single joint clearance, the error of angle and velocity are about $0.14^{\circ}$ and $1.75 \times 10^{-3} \% / \mathrm{s}$ respectively when all joints of the mechanism are considered clearance. Obviously, as the clearance size increases gradually, the error also increases. It can be seen from Figure 16 that when the clearance increases to $40 \mu \mathrm{m}$, the errors of rotation angle and rotation velocity are $0.043^{\circ}$ and $7.5 \times 10^{-4} \% \mathrm{~s}$, then the errors caused by clearances of all joint will be about $0.301^{\circ}$ and $5.25 \times 10^{-3} \%$ s. 


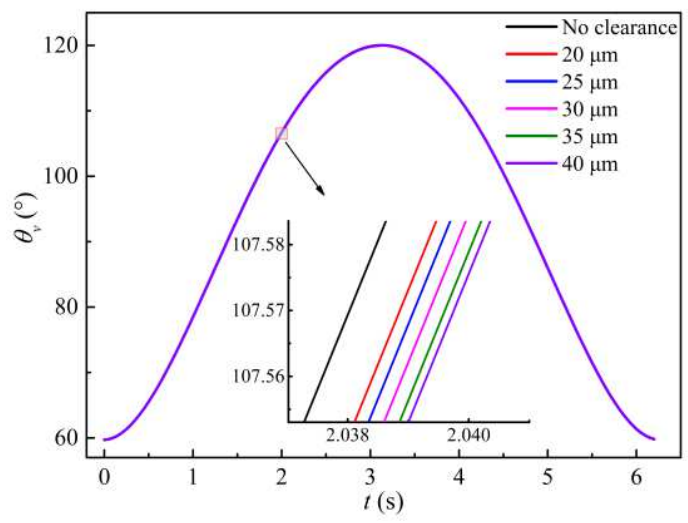

(a)

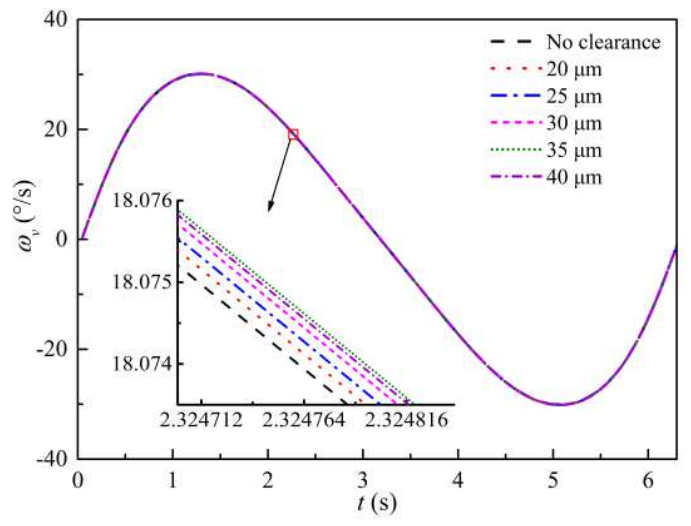

(b)

Fig. 16 Motion state of the vane with different clearance sizes: (a) rotation angle, (b) rotation velocity

As mentioned previously, there is a reversal of the vane direction in a complete cycle, and the contact area of the trunnion in the bushing changes accordingly, that is to say, the two surfaces in contact will separate and collide again. As shown in Figure 17, the contact forces, the damping forces, and the rigid forces oscillate in different degrees at the moment of collision. The contact forces $F_{c x}$ and $F_{c y}$ of different clearance sizes are almost equal, but when the clearance is greater than $30 \mu \mathrm{m}$, slight oscillations also occur in the continuous adjustment process, as shown in figures (a) and (b), which indicates that the large clearances may weaken the stability of the system. The damping forces are strongly dependent on the initial contact velocity, and the contact velocity increases with clearance size, so the effect on the damping forces is more obvious. In figures (c) and (d), when the vane is turned to $90^{\circ}$, the aerodynamic component force $F_{\text {airx }}$ changes direction, causing slight relative motion between trunnion and bushing, the relative motion velocity of large clearance is greater, so the damping force is higher than that of small clearance. When the two surfaces collide again after separation, the contact velocity is violent and the interference is serious, so the damping forces and rigid forces increase suddenly, as shown in figures (c) (f). In this process, the damping forces play a role in preventing excessive interference. Moreover, after reaching the maximum interference distance, the two surfaces begin to separate, and the damping forces also acts in the opposite direction to weaken the 
separation velocity. These effects can be confirmed by the positive and negative conversion of damping forces at the time of collision, as shown in the enlarged pictures in figures (c) and (d). Correspondingly, the interference of the asperities in the large clearances (35 $\mu \mathrm{m}$ and $40 \mu \mathrm{m})$ is relatively slight, so the rigid forces are smaller, as shown in the enlarged pictures of figures (e) and (f).

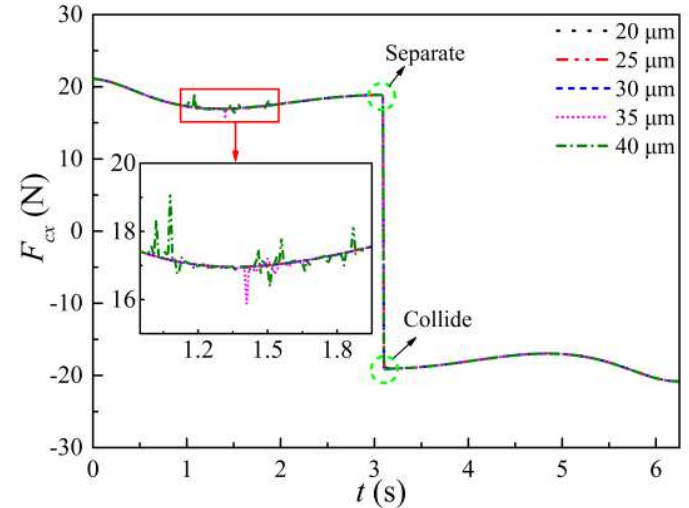

(a)

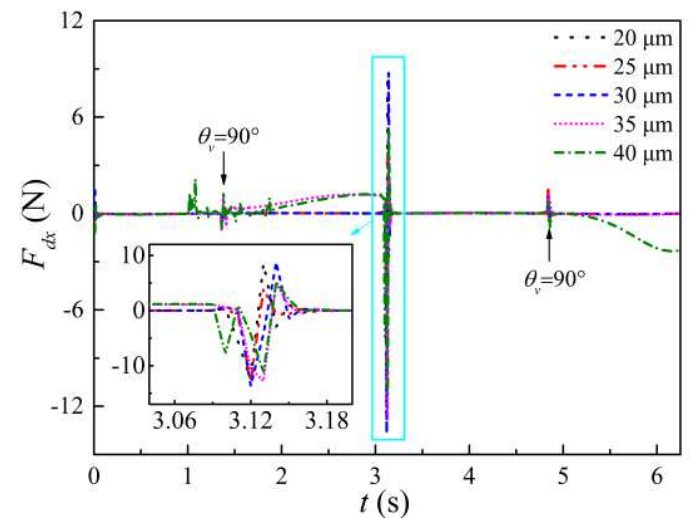

(c)

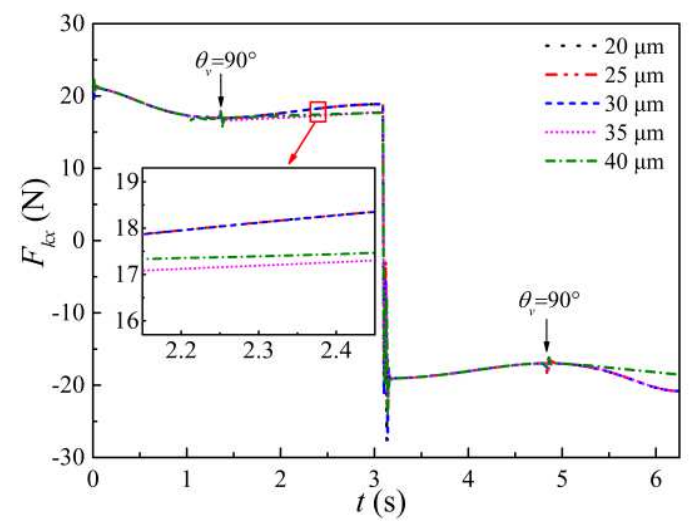

(e)

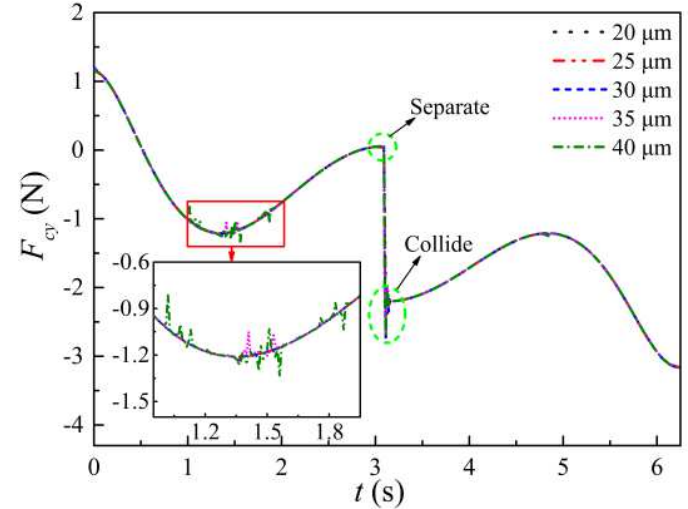

(b)

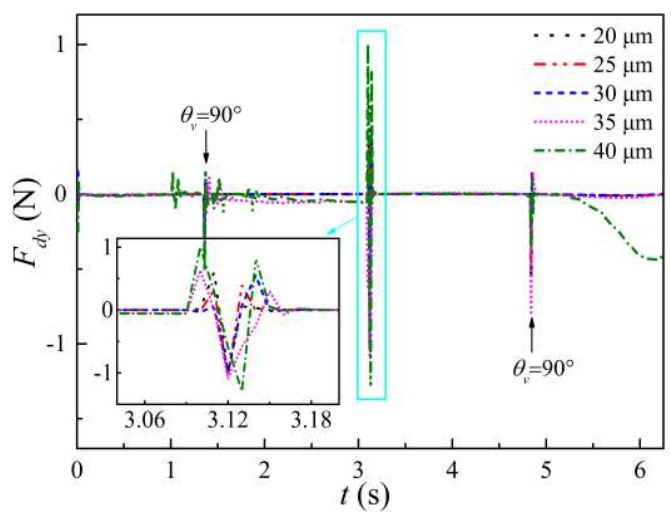

(d)

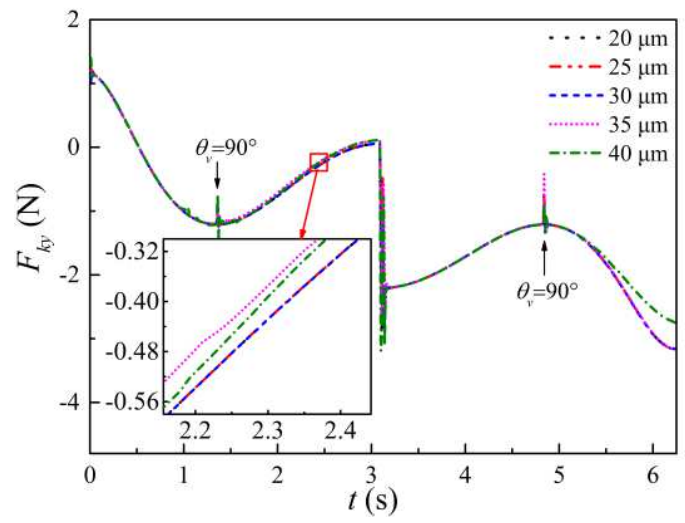

(f)

Fig. 17 Variation of forces with different clearance sizes: (a) and (b) for contact forces, (c) and (d) for damping forces, (e) and (f) for rigid forces 
The trajectory of the trunnion center $O_{j}$ and the deflection angle of the trunnion are shown in Figure 18. In a movement cycle, the deviation distances $e_{x}$ and $e_{y}$ increase with the increase of the clearance size, as shown in Figure 18 (a). Then, after the separation of the two contact surfaces, the velocity of the next collision in larger clearance will be greater, resulting in more significant damping force. Figure 18 (b) shows the deflection angle of the trunnion, which still follows the rule that the larger the clearance is, the larger the deflection angle is. In addition, the deflection angle $\varphi_{x}$ is always positive, which is the effect of aerodynamic drag, while the deflection angle $\varphi_{y}$ is alternating positive and negative, which is the effect of mechanism adjustment.

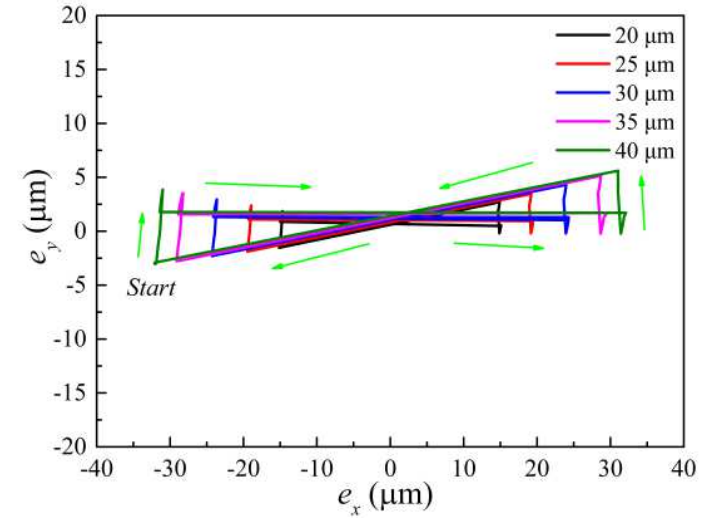

(a)

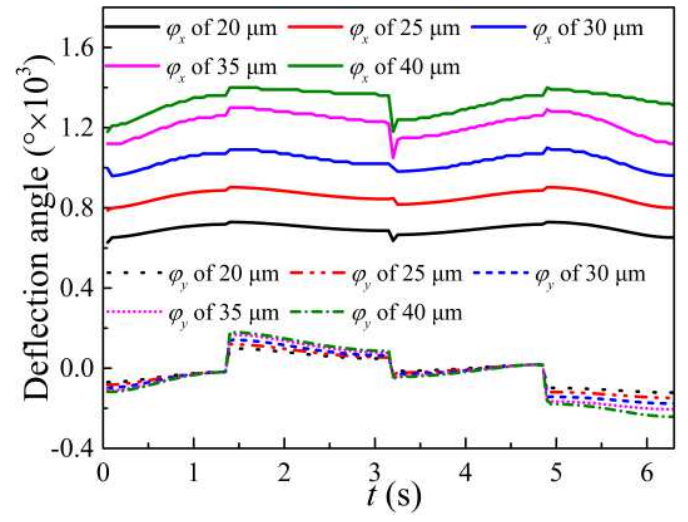

(b)

Fig.18 Attitude of the trunnion center with different clearance sizes: (a) trajectory, (b) deflection angle

The wear depth $h_{\text {wear }}$ of the bushing is shown in Figure 19. For each size of clearance, the wear of the mechanism running for 100 to 400 cycles is simulated. The wear mainly concentrated on the edge of the bushing, and gradually deepened with the accumulation of running times, which indicates the deflection of the trunnion keep its top and bottom in contact with the bushing. In addition, with the increase of the clearance, the wear becomes more and more serious. The mechanism operates 400 cycles under the clearance of $40 \mu \mathrm{m}$, and the maximum wear depth is $2 \times 10^{-4} \mu \mathrm{m}$, which is about 1.85 times of the clearance of $20 \mu \mathrm{m}$. 


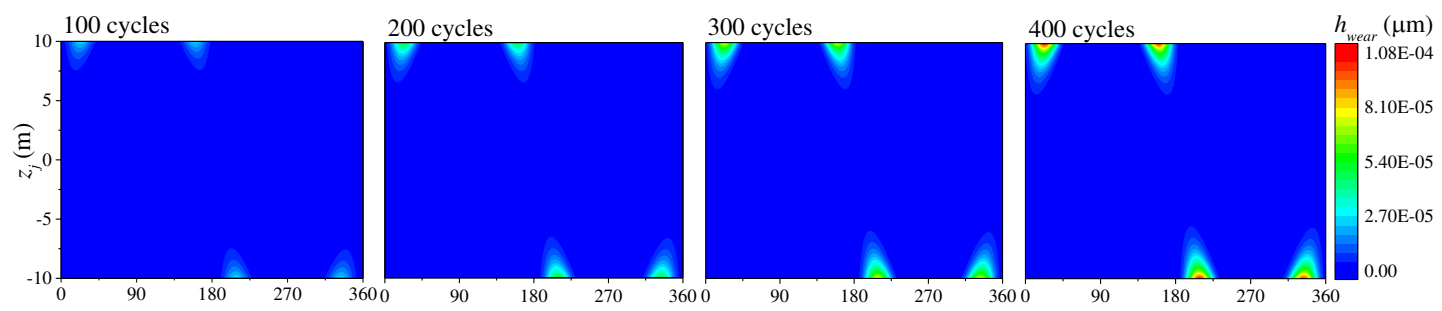

(a)

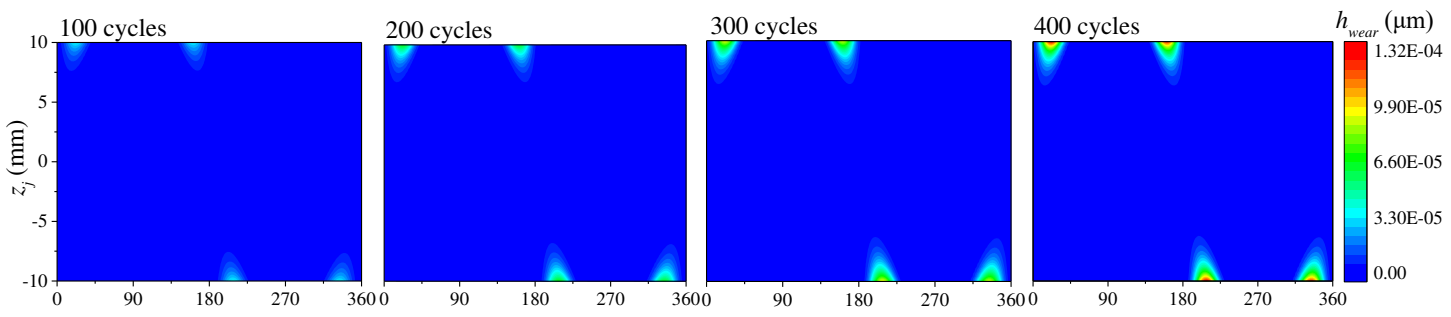

(b)

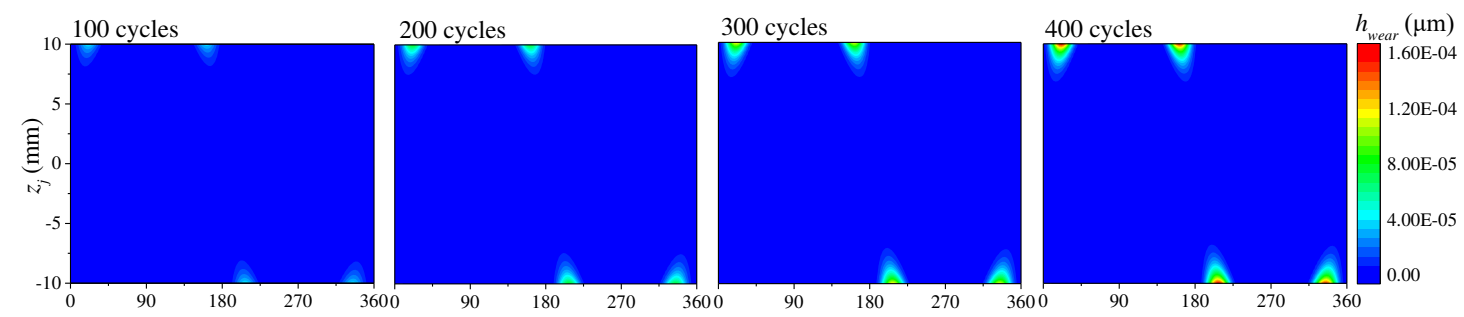

(c)
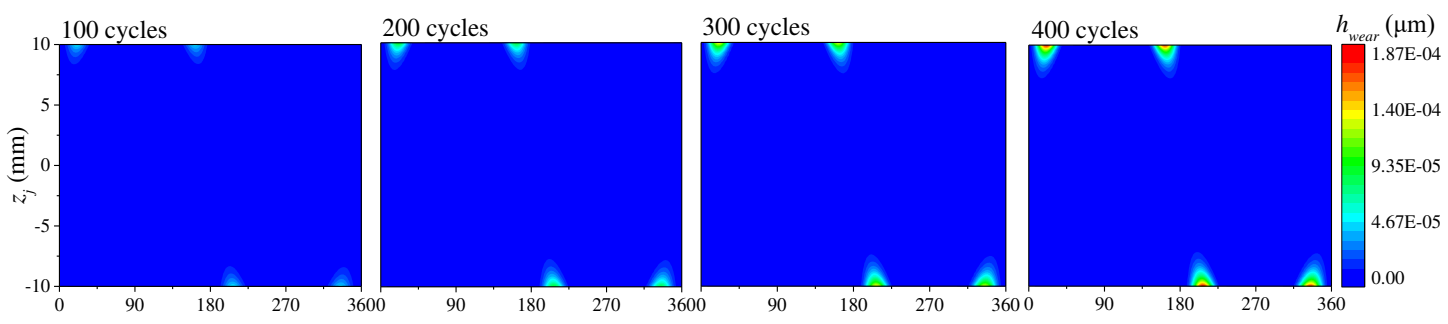

(d)

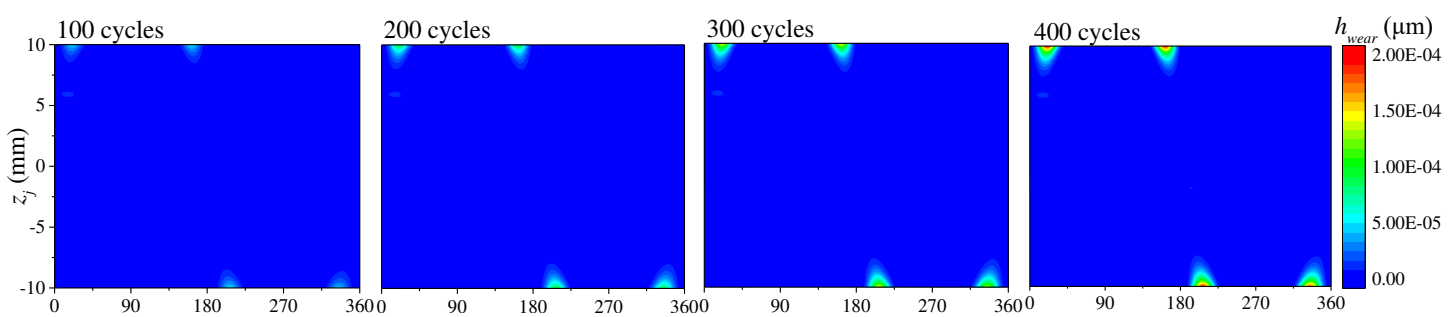

(e)

Circumferential position $\left(^{\circ}\right)$

Fig.19 Wear depth of the bushing with different clearance sizes: clearance = (a) $20 \mu \mathrm{m}$, (b) $25 \mu \mathrm{m}$, (c) $30 \mu \mathrm{m}$, (d) $35 \mu \mathrm{m}$, (e) $40 \mu \mathrm{m}$

\section{Conclusions}

In this paper, a rough surface damping contact model is proposed, and the spatial 
tribo-dynamics of the VSV mechanism is established based on this model. The main conclusions are drawn as follows:

(1) For dry friction pairs, the contact damping must be considered in the contact model, otherwise the calculation process will oscillate and not converge. This is quite different from the case with lubricating oil, that is to say, when there is lubricating oil, the contact damping is usually allowed to be omitted, mainly because the lubricating oil provides a more significant damping effect.

(2) During the operation of the VSV mechanism, the driving and aerodynamic drag lead to the deviation and deflection of the trunnion, so the contact area is concentrated on the edge of the bushing, and then wear occurs. In the dry friction pair of the trunnion and the bushing, the damping forces slow down the extrusion and separation of the asperities on the two surfaces, avoid the sudden variation of contact forces, and ensure the stability of the calculation process.

(3) With the increase of clearance size, the contact forces in the calculation process are more likely to fluctuate, and the distribution of the damping forces and the rigid forces is also affected. In addition, the increase of clearance reduces the adjustment accuracy and aggravates the wear.

\section{Acknowledgements}

This study was supported by the National Natural Science Foundation of China

(Grant No. 51875344), the Industry-University-Research cooperation project in Minhang District of Shanghai (Grant No. 2019MHC050), and the Shanghai Science and Technology Committee (Grant No. 19511133000).

\section{Compliance with ethical standards}

Conflict of interest: The authors declare that they have no conflict of interest.

\section{Appendix 1}

It can be concluded from Eqs. (12) and (13) that: 


$$
\left\{\begin{array}{c}
-l_{1} \sin \theta_{a}+l_{2} \sin \theta_{s z} \cos \theta_{s x} \cos \theta_{a}-l_{2} \cos \theta_{s Z} \sin \theta_{a}+l_{m} \cos \gamma=-l_{3} \sin \left(\theta_{b}-\varphi\right) \\
l_{2} \sin \theta_{s z} \sin \theta_{s x}+l_{4}=l_{4} \sin \theta_{v} \\
l_{1} \cos \theta_{a}+l_{2} \sin \theta_{s Z} \cos \theta_{s x} \sin \theta_{a}+l_{2} \cos \theta_{s z} \cos \theta_{a}+l_{m} \sin \gamma=l_{3} \cos \left(\theta_{b}-\varphi\right)
\end{array}\right.
$$

and from Eqs. (14) and (15), it can be concluded that:

$$
\left\{\begin{array}{c}
-\left(l_{3}+h_{2}\right) \sin \theta_{b}=l_{4} \cos \theta_{v} \\
l_{4}+h_{1}=l_{4} \sin \theta_{v} \\
\left(l_{3}+h_{2}\right) \cos \theta_{b}=l_{3}
\end{array}\right.
$$

where $h_{1}=l_{2} \sin \theta_{s z} \sin \theta_{s x}$, combining Eq. (54) and Eq. (55), we can get:

$$
\left\{\begin{array}{c}
\theta_{v}=\arccos \left(-\frac{l_{3} \sin \theta_{b}}{l_{4} \cos \theta_{b}}\right) \\
\theta_{s x}=\arctan \left[\frac{l_{4} \sin \theta_{v}-l_{4}}{l_{3} \sin \left(\theta_{a}-\theta_{b}+\varphi\right)-l_{m} \cos \left(\theta_{a}-\varphi\right)}\right] \\
\theta_{S Z}=\arcsin \left(\frac{l_{4} \sin \theta_{v}-l_{4}}{l_{2} \sin \theta_{s x}}\right)
\end{array}\right.
$$

\section{Appendix 2}

1. Coordinates of the mass center of the rods and the vane

$\operatorname{rod} 1:\left(x_{O 1}, y_{O 1}, z_{O 1}\right)=\left(l_{m} \cos \gamma-\frac{l_{1}}{2} \sin \theta_{a}, l_{4}, l_{m} \sin \gamma+\frac{l_{1}}{2} \cos \theta_{a}\right)$

$\operatorname{rod} 2:\left(x_{O 2}, y_{O 2}, z_{O 2}\right)=\left(\frac{e_{x}}{2}+l_{m} \cos \gamma-l_{1} \sin \theta_{a}+\frac{l_{2}}{2} \cos \theta_{a} \sin \theta_{s Z} \cos \theta_{s x}-\right.$

$\frac{l_{2}}{2} \sin \theta_{a} \cos \theta_{s Z}, \frac{e_{y}}{2}+l_{4}+\frac{l_{2}}{2} \sin \theta_{s Z} \sin \theta_{s x}, l_{m} \sin \gamma+l_{1} \cos \theta_{a}+\frac{l_{2}}{2} \cos \theta_{s Z} \cos \theta_{a}-$

$\left.\frac{l_{2}}{2} \sin \theta_{S Z} \sin \theta_{a}\right)$

$\operatorname{rad} 3:\left(x_{O 3}, y_{O 3}, z_{O 3}\right)=\left(\frac{e_{x}}{2}-\frac{l_{3}}{2} \cos \frac{\alpha}{2} \sin \left(\theta_{b}-\frac{\alpha}{2}\right), e_{y}+l_{4} \sin \theta_{d}, \frac{l_{3}}{2} \cos \frac{\alpha}{2} \cos \left(\theta_{b}-\frac{\alpha}{2}\right)\right)$

vane: $\left(x_{O v}, y_{O v}, z_{O v}\right)=\left(e_{x}+\frac{1}{2} l_{v} \sin \varphi_{y}, e_{y}+\frac{1}{2} l_{v} \sin \varphi_{x}, l_{3}-\frac{1}{2} l_{v}\right)$

Then the acceleration of mass centers in $X, Y$ and $Z$ directions can be obtained by the second derivative of coordinates:

$$
\begin{aligned}
& \ddot{x}_{O 1}=\frac{l_{1}}{2} \dot{\theta}_{a}{ }^{2} \sin \theta_{a}-\frac{l_{1}}{2} \ddot{\theta}_{a} \cos \theta_{a} \\
& \ddot{y}_{O 1}=0 \\
& \ddot{z}_{O 1}=-\frac{l_{1}}{2} \ddot{\theta}_{a} \sin \theta_{a}-l_{1} \dot{\theta}_{a}{ }^{2} \cos \theta_{a}
\end{aligned}
$$




$$
\begin{aligned}
& \ddot{x}_{O 2}=\frac{\ddot{e}_{x}}{2}-\ddot{\theta}_{a}\left(\sin \theta_{a} \sin \theta_{s z} \cos \theta_{s x}+\cos \theta_{s z} \cos \theta_{a}-l_{1} \sin \theta_{a}\right) \\
& -\dot{\theta}_{a}^{2}\left(\sin \theta_{s Z} \cos \theta_{s x} \cos \theta_{a}-\sin \theta_{a} \cos \theta_{s z}-l_{1} \cos \theta_{a}\right) \\
& +\frac{l_{2}}{2}\left[\ddot{\theta}_{s Z}\left(\cos \theta_{s Z} \cos \theta_{s x} \cos \theta_{a}+\sin \theta_{a} \sin \theta_{s Z}\right)\right. \\
& -\dot{\theta}_{s Z} \dot{\theta}_{a}\left(2 \sin \theta_{a} \cos \theta_{s Z} \cos \theta_{s x}-\cos \theta_{a} \sin \theta_{S Z}\right) \\
& +\dot{\theta}_{s x} \dot{\theta}_{a}\left(2 \sin \theta_{a} \sin \theta_{s z} \sin \theta_{s x}\right)-\left(\dot{\theta}_{s z}{ }^{2}+\dot{\theta}_{s x}{ }^{2}\right) \sin \theta_{s z} \cos \theta_{s x} \cos \theta_{a} \\
& +\dot{\theta}_{s 1 z}^{2} \sin \theta_{a} \cos \theta_{s z}-2 \dot{\theta}_{s 1 z} \dot{\theta}_{s x} \cos \theta_{s z} \sin \theta_{s x} \cos \theta_{a} \\
& \left.-\ddot{\theta}_{s x} \sin \theta_{s z} \sin \theta_{s x} \cos \theta_{a}\right] \\
& \ddot{y}_{O 2}=\frac{\ddot{e}_{y}}{2}+\frac{l_{2}}{2}\left(\ddot{\theta}_{s z} \cos \theta_{s z} \sin \theta_{s x}-\left(\dot{\theta}_{s z}{ }^{2}+\dot{\theta}_{s x}{ }^{2}\right) \sin \theta_{s z} \sin \theta_{s x}+2 \dot{\theta}_{s Z} \dot{\theta}_{s x} \cos \theta_{s z} \cos \theta_{s x}\right. \\
& \left.+\ddot{\theta}_{s x} \sin \theta_{s z} \cos \theta_{s x}\right) \\
& \ddot{z}_{O 2}=\ddot{\theta}_{a}\left[-l_{1} \sin \theta_{a}-\frac{l_{2}}{2} \sin \left(\theta_{S Z}+\theta_{a}\right)\right]-\dot{\theta}_{a}{ }^{2}\left[l_{1} \cos \theta_{a}+\frac{l_{2}}{2} \cos \left(\theta_{S Z}+\theta_{a}\right)\right] \\
& -\frac{l_{2}}{2} \ddot{\theta}_{s z} \sin \left(\theta_{s z}+\theta_{a}\right)-\frac{l_{2}}{2} \dot{\theta}_{s z}{ }^{2} \cos \left(\theta_{s z}+\theta_{a}\right)-l_{2} \dot{\theta}_{s z} \dot{\theta}_{a} \cos \left(\theta_{s z}+\theta_{a}\right) \\
& \ddot{x}_{O 3}=\frac{\ddot{e}_{x}}{2}-\frac{l_{3}}{2} \cos \frac{\alpha}{2}\left[\ddot{\theta}_{b} \cos \left(\theta_{b}-\frac{\alpha}{2}\right)-\dot{\theta}_{b}^{2} \sin \left(\theta_{b}-\frac{\alpha}{2}\right)\right] \\
& \ddot{y}_{O 3}=\ddot{e}_{y}+l_{4}\left(\ddot{\theta}_{d} \cos \theta_{d}-\dot{\theta}_{d}{ }^{2} \sin \theta_{d}\right) \\
& \ddot{z}_{03}=-\frac{l_{3}}{2} \ddot{\theta}_{b} \cos \frac{\alpha}{2} \sin \left(\theta_{b}-\frac{\alpha}{2}\right)-\frac{l_{3}}{2} \dot{\theta}_{b}{ }^{2} \cos \frac{\alpha}{2} \cos \left(\theta_{b}-\frac{\alpha}{2}\right) \\
& \ddot{x}_{O v}=\ddot{e}_{x}+\frac{l_{v}}{2}\left(\ddot{\varphi}_{y} \cos \varphi_{y}-\dot{\varphi}_{y}{ }^{2} \sin \varphi_{y}\right) \\
& \ddot{y}_{O v}=\ddot{e}_{y}+\frac{l_{v}}{2}\left(\ddot{\varphi}_{x} \cos \varphi_{x}-\dot{\varphi}_{x}{ }^{2} \sin \varphi_{x}\right) \\
& \ddot{z}_{O v}=0
\end{aligned}
$$

Therefore, the inertia forces and moments in Eqs. (19) (42) can be expressed as

$$
\begin{aligned}
& F_{1 x i}=-m_{1} \cdot \ddot{x}_{O 1} \\
& F_{1 z i}=-m_{1} \cdot \ddot{z}_{O 1} \\
& M_{1 y i}=-J_{1} \cdot \ddot{\theta}_{a} \\
& F_{2 x i}=-m_{2} \cdot \ddot{x}_{O 2} \\
& F_{2 y i}=-m_{2} \cdot \ddot{y}_{O 2} \\
& F_{2 z i}=-m_{2} \cdot \ddot{z}_{O 2} \\
& M_{2 x i}=-J_{2} \cdot\left[\left(2 \dot{\theta}_{s z} \dot{\theta}_{s x}+\ddot{\theta}_{s x} \theta_{s z}\right) \cos \theta_{s x}+\left(\ddot{\theta}_{s z}-\theta_{s z} \dot{\theta}_{s x}{ }^{2}\right) \sin \theta_{s x}\right]
\end{aligned}
$$




$$
\begin{aligned}
& M_{2 y i}=-J_{2} \cdot\left[\left(2 \dot{\theta}_{s z} \dot{\theta}_{s x}+\ddot{\theta}_{s x} \theta_{s z}\right) \sin \theta_{s x}-\left(\ddot{\theta}_{s z}-\theta_{s z} \dot{\theta}_{s x}{ }^{2}\right) \cos \theta_{s x}\right] \\
& M_{2 z i}=-J_{2} \cdot\left\{\left[2\left(\dot{\theta}_{s z}+\dot{\theta}_{a}\right) \dot{\theta}_{s x}+\left(\ddot{\theta}_{s z}+\ddot{\theta}_{a}\right) \theta_{s x}\right] \cos \left(\theta_{s z}+\theta_{a}\right)\right. \\
& \left.\quad+\left[\ddot{\theta}_{s x}-\theta_{s x}\left(\dot{\theta}_{s z}+\dot{\theta}_{a}\right)^{2}\right] \sin \left(\theta_{s z}+\theta_{a}\right)\right\} \\
& F_{3 x i}=-m_{3} \cdot \ddot{x}_{O 3} \\
& F_{3 y i}=-m_{3} \cdot \ddot{y}_{O 3} \\
& F_{3 z i}=-m_{3} \cdot \ddot{z}_{O 3} \\
& M_{3 y i}=-J_{3} \cdot \ddot{\theta}_{b} \\
& F_{v x i}=-m_{v} \cdot \ddot{e}_{x} \\
& F_{v y i}=-m_{v} \cdot e_{y} \\
& M_{v x i}=-J_{v} \cdot \ddot{\varphi}_{x} \\
& M_{v y i}=-J_{v} \cdot \ddot{\theta}_{y} \\
& M_{v z i}=-J_{v} \cdot \ddot{\theta}_{v}
\end{aligned}
$$

2. The detailed expressions of intermediate variables in Eq. (46):

$$
\begin{aligned}
A_{11}= & -m_{v} \cdot \operatorname{Loc}_{1}-\frac{m_{3}}{2} \cdot \operatorname{Loc}_{2}-\frac{m_{2}}{2} \cdot \operatorname{Loc}_{3} \\
A_{12}= & -\frac{m_{2}}{2} \cdot \operatorname{Loc}_{4}-\frac{m_{2}}{2}-m_{v} \\
A_{13}= & -\frac{m_{v} l_{v}}{2} \cos \varphi_{x} \\
A_{14}= & -\frac{m_{v} l_{v}}{2} \cdot \operatorname{Loc}_{1} \cdot \cos \varphi_{y} \\
B_{11}= & -\frac{m_{v} l_{v}}{2} \dot{\varphi}_{y}{ }^{2} \sin \varphi_{y} \cdot \operatorname{Loc}_{1}+m_{3} \cdot \operatorname{ParX}_{3} \cdot \operatorname{Loc}_{2}+m_{2} \cdot \operatorname{ParX}_{2} \cdot \operatorname{Loc}_{3}+m_{2} \cdot \operatorname{Pary}_{2} \cdot \operatorname{Loc}_{4} \\
\quad & \quad+m_{3} \cdot \operatorname{Pary}_{3}-\frac{m_{v} l_{v}}{2} \dot{\varphi}_{x}{ }^{2} \operatorname{Sin} \varphi_{x}-\operatorname{Loc}_{5}-F_{c y}-F_{\text {airy }}
\end{aligned}
$$$$
A_{21}=0
$$$$
A_{22}=-\frac{m_{v} l_{v}}{2}
$$$$
A_{23}=-\frac{m_{v} l_{v}^{2}}{4} \cos \varphi_{x}-J_{v}
$$$$
A_{24}=0
$$$$
B_{21}=-\frac{m_{v} l_{v}^{2}}{4} \dot{\varphi}_{x}{ }^{2} \sin \varphi_{x}-M_{t c x}-M_{j c x}-\frac{l_{v}}{2} F_{\text {airy }}
$$$$
A_{31}=-\frac{m_{v} l_{v}}{2}
$$ 


$$
\begin{aligned}
& A_{32}=0 \\
& A_{33}=0 \\
& A_{34}=-\frac{m_{v} l_{v}{ }^{2}}{4} \cos \varphi_{y}-J_{v} \\
& B_{31}=-\frac{m_{v} l_{v}^{2}}{4} \dot{\varphi}_{y}{ }^{2} \sin \varphi_{y}-M_{t c y}-M_{j c y}-\frac{l_{v}}{2} F_{a i r x} \\
& A_{41}=-m_{v} X_{s_{3}}^{O_{j}} \cdot L o c_{1}-\frac{m_{3}}{2} X_{s_{3}}^{O_{j}} \cdot \operatorname{Loc}_{2}-\frac{m_{2}}{2} X_{s_{3}}^{O_{j}} \cdot \operatorname{Loc}_{3}-m_{v} Y_{s_{3}}^{O_{j}} \\
& A_{42}=-\frac{m_{2}}{2} X_{s_{3}}^{O_{j}} \cdot L o c_{4}-\frac{m_{3}}{2} X_{S_{3}}^{O_{j}} \\
& A_{43}=0 \\
& A_{44}=-\frac{m_{v} l_{v}}{2} X_{s_{3}}^{O_{j}} \cos \varphi_{y} \cdot \operatorname{Loc}_{1}-\frac{m_{v} l_{v}}{2} Y_{s_{3}}^{O_{j}} \cos \varphi_{y} \\
& B_{41}=-\frac{m_{v} l_{v}}{2} X_{s_{3}}^{O_{j}} \dot{\varphi}_{y}{ }^{2} \sin \varphi_{y} \cdot \operatorname{Loc}_{1}+m_{3} X_{s_{3}}^{O_{j}} \cdot \operatorname{Parx}_{3} \cdot \operatorname{Loc}_{2}+m_{2} X_{s_{3}}^{O_{j}} \cdot \operatorname{Parx}_{2} \cdot \operatorname{Loc}_{3}+m_{2} X_{s_{3}}^{O_{j}} \\
& \cdot \operatorname{Pary}_{2} \cdot \operatorname{Loc}_{4}+m_{3} X_{s_{3}}^{O_{j}} \cdot \operatorname{Pary}_{3}-m_{v} Y_{s_{3}}^{O_{j}} \dot{\varphi}_{y}{ }^{2} \sin \varphi_{y}-X_{s_{3}}^{O_{j}} \cdot \operatorname{Loc}_{5}-Y_{s_{3}}^{O_{j}} F_{c x} \\
& +J_{v} \alpha_{4}-M_{j f}-M_{t f}-M_{\text {air }}
\end{aligned}
$$

where

$$
\begin{aligned}
& L_{o c 1}=\frac{\left(Y_{s 1}^{O_{2}}-Y_{s 2}^{O_{2}}\right)\left(Z_{b}^{O_{3}}-Z_{s 3}^{O_{3}}\right)}{\left(Z_{s 2}^{O_{3}}-Z_{b}^{O_{3}}\right)\left(X_{s 1}^{O_{2}}-X_{s 2}^{O_{2}}\right)-\left(X_{b}^{O_{3}}-X_{s 2}^{O_{3}}\right)\left(Z_{s 2}^{O_{2}}-Z_{s 1}^{O_{2}}\right)} \\
& L_{o c 2}=\frac{Z_{b}^{O_{3}}\left(Y_{s 1}^{O_{2}}-Y_{s 2}^{O_{2}}\right)}{\left(Z_{s 2}^{O_{3}}-Z_{b}^{O_{3}}\right)\left(X_{s 1}^{O_{2}}-X_{s 2}^{O_{2}}\right)-\left(X_{b}^{O_{3}}-X_{s 2}^{O_{3}}\right)\left(Z_{s 2}^{O_{2}}-Z_{s 1}^{O_{2}}\right)} \\
& L_{o c 3}=\frac{Z_{s 1}^{O_{2}}\left(X_{s 2}^{O_{3}}-X_{b}^{O_{3}}\right)\left(Y_{s 1}^{O_{2}}-Y_{s 2}^{O_{2}}\right)}{\left(Z_{b}^{O_{3}}-Z_{s 2}^{O_{3}}\right)\left(X_{s 2}^{O_{2}}-X_{s 1}^{O_{2}}\right)^{2}-\left(X_{s 2}^{O_{2}}-X_{s 1}^{O_{2}}\right)\left(X_{b}^{O_{3}}-X_{s 2}^{O_{3}}\right)\left(Z_{s 2}^{O_{2}}-Z_{s 1}^{O_{2}}\right)}-\frac{Y_{s 1}^{O_{2}}}{\left(X_{s 1}^{O_{2}}-X_{s 2}^{O_{2}}\right)} \\
& L_{o c 4}=\frac{X_{s 1}^{O_{2}}}{\left(X_{s 1}^{O_{2}}-X_{s 2}^{O_{2}}\right)} \\
& L_{o c 5}
\end{aligned}
$$

$$
=\frac{\left(Y_{s 1}^{O_{2}}-Y_{s 2}^{O_{2}}\right)\left[\left(Z_{b}^{O_{3}}-Z_{s 3}^{O_{3}}\right)\left(F_{a i r x}+F_{c x}\right)+M_{3 y i}+Z_{b}^{O_{3}}\left(G_{3}-F_{3 z i}\right)+\frac{X_{s 1}^{O_{2}}\left(G_{2}-F_{2 z i}\right)+M_{2 y i}}{\left(X_{s 2}^{O_{2}}-X_{s 1}^{O_{2}}\right)}\left(X_{s 2}^{O_{3}}-X_{b}^{O_{3}}\right)\right]}{\left(X_{s 1}^{O_{2}}-X_{s 2}^{O_{2}}\right)\left(Z_{s 2}^{O_{3}}-Z_{b}^{O_{3}}\right)-\left(X_{b}^{O_{3}}-X_{s 2}^{O_{3}}\right)\left(Z_{s 2}^{O_{2}}-Z_{s 1}^{O_{2}}\right)}
$$




$$
\begin{aligned}
& P_{a r x 2}=-\ddot{\theta}_{a}\left(\sin \theta_{a} \sin \theta_{s Z} \cos \theta_{s x}+\cos \theta_{s Z} \cos \theta_{a}-l_{1} \sin \theta_{a}\right) \\
& -\dot{\theta}_{a}^{2}\left(\sin \theta_{S Z} \cos \theta_{s x} \cos \theta_{a}-\sin \theta_{a} \cos \theta_{s Z}-l_{1} \cos \theta_{a}\right) \\
& +\frac{l_{2}}{2}\left[\ddot{\theta}_{s z}\left(\cos \theta_{s z} \cos \theta_{s 1 x} \cos \theta_{a}+\sin \theta_{a} \sin \theta_{s z}\right)\right. \\
& -\dot{\theta}_{s z} \dot{\theta}_{a}\left(2 \sin \theta_{a} \cos \theta_{s z} \cos \theta_{s x}-\cos \theta_{a} \sin \theta_{s z}\right) \\
& +\dot{\theta}_{s x} \dot{\theta}_{a}\left(2 \sin \theta_{a} \sin \theta_{s z} \sin \theta_{s x}\right)-\left(\dot{\theta}_{s z}{ }^{2}+\dot{\theta}_{s x}{ }^{2}\right) \sin \theta_{s z} \cos \theta_{s x} \cos \theta_{a} \\
& +\dot{\theta}_{s z}^{2} \sin \theta_{a} \cos \theta_{s Z}-2 \dot{\theta}_{s z} \dot{\theta}_{s x} \cos \theta_{s z} \sin \theta_{s x} \cos \theta_{a} \\
& \left.-\ddot{\theta}_{s x} \sin \theta_{s z} \sin \theta_{s x} \cos \theta_{a}\right] \\
& P_{\text {ary } 2}=\frac{l_{2}}{2}\left[\ddot{\theta}_{s z} \cos \theta_{s z} \sin \theta_{s x}-\left(\dot{\theta}_{s z}{ }^{2}+\dot{\theta}_{s x}{ }^{2}\right) \sin \theta_{s z} \sin \theta_{s x}+2 \dot{\theta}_{s z} \dot{\theta}_{s x} \cos \theta_{s z} \cos \theta_{s x}\right. \\
& \left.+\ddot{\theta}_{s x} \sin \theta_{s z} \cos \theta_{s x}\right] \\
& P_{\text {arx } 3}=-\frac{l_{3}}{2} \cos \frac{\alpha}{2}\left[\ddot{\theta}_{b} \cos \left(\theta_{b}-\frac{\alpha}{2}\right)-\dot{\theta}_{b}{ }^{2} \sin \left(\theta_{b}-\frac{\alpha}{2}\right)\right] \\
& P_{\text {ary3 }}=l_{4}\left(\ddot{\theta}_{v} \cos \theta_{v}-\dot{\theta}_{v}^{2} \sin \theta_{v}\right)
\end{aligned}
$$

3. The global coordinates of the joints relative to the mass center of the rods are as follows:

For the mass center $O_{1}$ :

$A:\left(X_{a}^{O_{1}}, Y_{a}^{O_{1}}, Z_{a}^{O_{1}}\right)=\left(\frac{l_{1}}{2} \sin \theta_{a}, 0,-\frac{l_{1}}{2} \cos \theta_{a}\right)$

$S_{l}:\left(X_{s_{1}}^{O_{1}}, Y_{s_{1}}^{O_{1}}, Z_{s_{1}}^{O_{1}}\right)=\left(-X_{a}^{O_{1}},-Y_{a}^{O_{1}},-Z_{a}^{O_{1}}\right)$

For the mass center $\mathrm{O}_{2}$ :

$S_{1}:\left(X_{s_{1}}^{O_{2}}, Y_{s_{1}}^{O_{2}}, Z_{s_{1}}^{O_{2}}\right)=\left(\frac{l_{2}}{2} \cos \theta_{S Z} \sin \theta_{a}-\right.$

$\left.\frac{l_{2}}{2} \sin \theta_{s Z} \cos \theta_{s x} \cos \theta_{a},-\frac{l_{2}}{2} \sin \theta_{s Z} \sin \theta_{s x},-\frac{l_{2}}{2} \cos \theta_{s Z} \cos \theta_{a}-\frac{l_{2}}{2} \sin \theta_{s Z} \cos \theta_{s x} \sin \theta_{a}\right)$

$S_{2}:\left(X_{s_{2}}^{O_{2}}, Y_{s_{2}}^{O_{2}}, Z_{s_{2}}^{O_{2}}\right)=\left(-X_{s_{1}}^{O_{2}},-Y_{s_{1}}^{O_{2}},-Z_{s_{1}}^{O_{2}}\right)$

For the mass center $\mathrm{O}_{3}$ :

$S_{2}:\left(X_{S_{2}}^{O_{3}}, Y_{S_{2}}^{O_{3}}, Z_{s_{2}}^{O_{3}}\right)=\left(l_{3} \cos \left(\theta_{b}-\varphi\right)-\frac{l_{3}}{2} \cos \frac{\varphi}{2} \cos \left(\theta_{b}-\frac{\varphi}{2}\right), 0, l_{3} \sin \left(\theta_{b}-\varphi\right)-\right.$ $\left.\frac{l_{3}}{2} \cos \frac{\varphi}{2} \sin \left(\theta_{b}-\frac{\varphi}{2}\right)\right)$

$B:\left(X_{b}^{O_{3}}, Y_{b}^{O_{3}}, Z_{b}^{O_{3}}\right)=\left(-\frac{l_{3}}{2} \cos \frac{\varphi}{2} \cos \left(\theta_{b}-\frac{\varphi}{2}\right), 0,-\frac{l_{3}}{2} \cos \frac{\varphi}{2} \sin \left(\theta_{b}-\frac{\varphi}{2}\right)\right)$

$S_{3}:\left(X_{s_{3}}^{O_{3}}, Y_{s_{3}}^{O_{3}}, Z_{S_{3}}^{O_{3}}\right)=\left(l_{3} \cos \theta_{b}-\frac{l_{3}}{2} \cos \frac{\varphi}{2} \cos \left(\theta_{b}-\frac{\varphi}{2}\right), 0, l_{3} \sin \theta_{b}-\frac{l_{3}}{2} \cos \frac{\varphi}{2} \sin \left(\theta_{b}-\frac{\varphi}{2}\right)\right)$ 
For the center of the trunnion $O_{j}$ :

$S_{3}:\left(X_{s 3}^{O_{j}}, Y_{s 3}^{O_{j}}, Z_{s 3}^{O_{j}}\right)=\left(l_{4} \cos \theta_{v}, l_{4} \sin \theta_{v}, \frac{l_{z}}{2}\right)$

\section{References}

1. Goldsmith, W.: Impact: The theory and physical behavior of colliding solids. E. Arnold, London (1960)

2. Hunt, K.H., Crossley, F.R.E.: Coefficient of restitution interpreted as damping in vibroimpact. ASME J. Appl. Mech. 42(2), 440-445 (1975)

3. Lee, T.W., Wang, A.: On the dynamics of intermittent-motion mechanisms. Part 1: dynamic model and response. J. Mech. Design. 105(3), 534-540 (1983)

4. Lankarani, H.M., Nikravesh, P.E.: A contact force model with hysteresis damping for impact analysis of multibody systems. J. Mech. Design. 112(3), 369-376 (1990)

5. Zhao, B., Zhang, Z.N., Fang, C.C., Dai, X.D., Xie, Y.B.: Modeling and analysis of planar multibody system with mixed lubricated revolute joint. Tribol. Int. 98, 229-241 (2016)

6. Chen, X., Jiang, S., Deng, Y., Wang, Q.: Dynamics analysis of 2-DOF complex planar mechanical system with joint clearance and flexible links. Nonlinear Dyn. 93(3), 1009-1034 (2018)

7. Li, Y., Wang, C., Huang, W.: Rigid-flexible-thermal analysis of planar composite solar array with clearance joint considering torsional spring, latch mechanism and attitude controller. Nonlinear Dyn. 96(3), 2031-2053 (2019)

8. Sun, D., Zhang, B., Liang, X., Shi, Y., Suo, B.: Dynamic analysis of a simplified flexible manipulator with interval joint clearances and random material properties. Nonlinear Dyn. 98(2), 1049-1063 (2019)

9. Yan, S.Z., Xiang, W.W.K., Zhang, L.: A comprehensive model for 3D revolute joints with clearances in mechanical systems. Nonlinear Dyn. 80(1-2), 309-328 (2015)

10. Xiang, W.W.K., Yan, S.Z., Wu, J.N.: Dynamic analysis of planar mechanical systems considering stick-slip and Stribeck effect in revolute clearance joints. Nonlinear Dyn. 95(1), 321-341 (2019)

11. Lankarani, H.: Canonical equations of motion and estimation of parameters in the analysis of impact problems. University of Arizona, Tucson, Arizona (1988) 
12. Greenwood, J.A., Williamson, J.B.P.P.: Contact of nominally flat surfaces. P. Roy. Soc. A-Math. 295, 300-319 (1966)

13. Greenwood, J.A., Tripp, J.H.: The elastic contact of rough spheres. J. Appl. Mech. 34(1), 153$159(1967)$

14. Chang, W.R., Etsion, I., Bogy, D.B.: An elastic-plastic model for the contact of rough surfaces. ASME J. Tribol. 109(2), 257-263

15. Kogut, L., Etsion, I.: Elastic-plastic contact analysis of a sphere and a rigid flat. J. Appl. Mech. 69(5), 657-662 (2002)

16. Chen, Y.H., Zhang, X.L., Wen, S.H., Lan, G.S.: Fractal model for normal contact damping of joint surface considering elastoplastic phase. Journal of Mechanical Engineering. 55(16), 58-68 (2019)

17. Pan, W.J., Li, X.P., Guo, N., Yang, Z.M., Sun, Z.N.: Three-dimensional fractal model of normal contact damping of dry-friction rough surface. Adv. Mech. Eng. 9(3), 1-11 (2017)

18. Wirkowski, P.: Influence of the incorrect settings of axial compressor inlet variable stator vanes on gas turbine engine work parameters. J. Kones. 19, 483-489 (2012)

19. Wang, Z.T., Li, J., Fan, K., Li, S.Y.: The off-design performance simulation of marine gas turbine based on optimum scheduling of variable stator vanes. Math. Probl. Eng. 8, 1-11 (2017)

20. Sugimoto, T., Nagai, K., Ryu, M., Tanaka, R., Kimura, T.: A review of L20A engine design and field operating experience. In: Turbo Expo: Power for Land, Sea, and Air. 537-541 (2004)

21. Striebing, D.R., Stanford, M.K., DellaCorte, C., Rossi, A.M.: Tribological performance of PM300 solid lubricant bushings for high temperature applications. NASA/TM-2007-214819 (2007)

22. Zhang, Z., Xiao, Y., Xie, Y.H., Su, Z.Q.: Effects of contact between rough surfaces on the dynamic responses of bolted composite joints: Multiscale modeling and numerical simulation. Compos. Struct. 211, 13-23 (2019).

23. Pan, W.J, Li, H.S., Qu, H.Y., Ling, L.Y., Wang, L.L.: Investigation of tangential contact damping of rough surfaces from the perspective of viscous damping mechanism. ASME J.Tribol. 143(4), $041501(2021)$

24. Yu, J.P., Sun, J.M., Ji, F.S., Zhang, S., Zhang, Z.J.: Motion analysis and optimization of jointly adjusting mechanism of aero-engine stator vane. Journal of Aerospace Power. 34(6), 1193-1200 
(2019)

25. Reitenbach, S., Schnoes, M., Becker, R.G., Otten, T.: Optimization of compressor variable geometry settings using multi-fidelity simulation. In: Turbo Expo: Power for Land, Sea, and Air. V02CT45A010 (2015)

26. Wang, J.Y., Yang, R.F., Wang, Z.Q., Hu, J.: Effect of location of penny platform in variable stator vane on endwall flow. Journal of Aerospace Power. 27(11), 2585-2591 (2012)

27. Tang, Y.Y., Guo, W.Z.: Global dimensional optimization for the design of adjusting mechanism of variable stator vanes. Journal of Mechanical Engineering. 56(11), 26-36 (2020)

28. Riesland, D.: Aircraft engine analysis using ADAMS. In: European ADAMS User Conference, New Yoik, 1-7 (2000)

29. Kogut, L., Etsion, I.: A finite element based elastic-plastic model for the contact of rough surfaces. Trib. Trans. 46(3), 383-390 (2003)

30. Zhang, R., Meng, X.H., Lyu, B.G., Sun, K.: A deterministic FE contact analysis of 3D rough surfaces with textures and comparison with classic statistical contact models. Sci. China Tech. Sci. (2020). https://doi.org/10.1007/s11431-019-1536-6

31. Archard, J.: Contact and rubbing of flat surfaces. J. Appl. Phys. 24(8), 981-988 (1953)

32. Cash, J.: Modified extended backward differentiation formulae for the numerical solution of stiff initial value problems in ODEs and DAEs. J. Comput. Appl. Math. 125(1-2), 117-130 (2000)

33. Li, R., Meng, X.H., Xie, Y.B.: A new coupling tribodynamic model of crosshead slipper-guide system and piston skirt-liner system of low-speed marine diesel engines. Tribol. Int. 117, 189$205(2018)$

34. Liu, R.C., Meng, X.H., Cui, Y.: Influence of numerous start-ups and stops on tribological performance evolution of engine main bearings. Int. J. Engine Res. 21(8), 1362-1380 (2020)

35. Patir, N., Cheng, H.S.: An average flow model for determining effects of three-dimensional roughness on partial hydrodynamic lubrication. J. Lubr. Technol. 100, 12-17 (1978)

36. Patir, N., Cheng, H.S.: Application of average flow model to lubrication between rough sliding surfaces. J. Lubr. Technol. 101, 220-229 (1979)

37. Wu, C.W., Zheng, L.Q.: An average reynolds equation lubrication with a contact factor. ASME J.Tribol. 111, 188-191 (1989) 


\section{Figures}

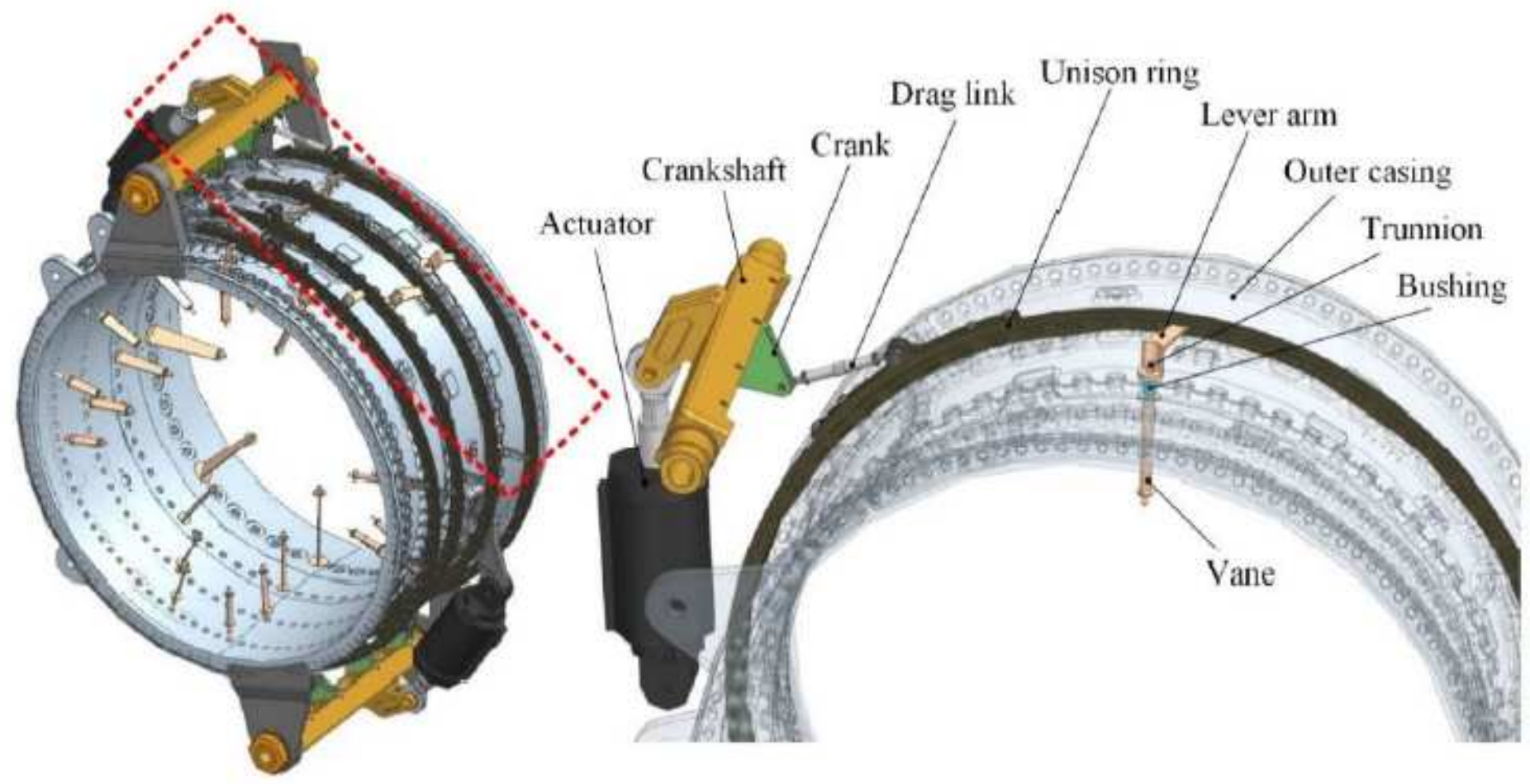

Figure 1

Schematic diagram of the VSV mechanism

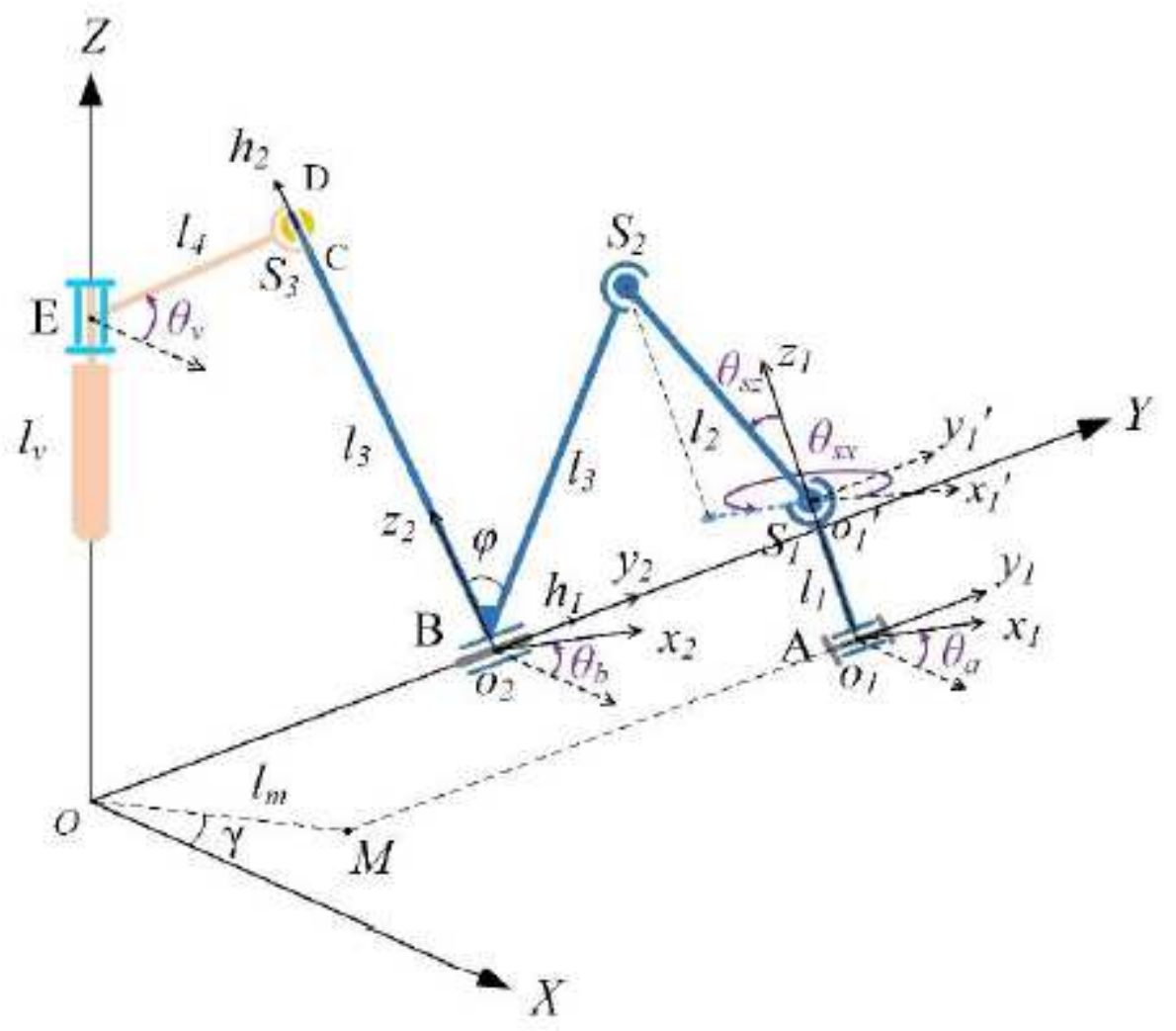


Figure 2

Schematic diagram of spatial multi-rod mechanism

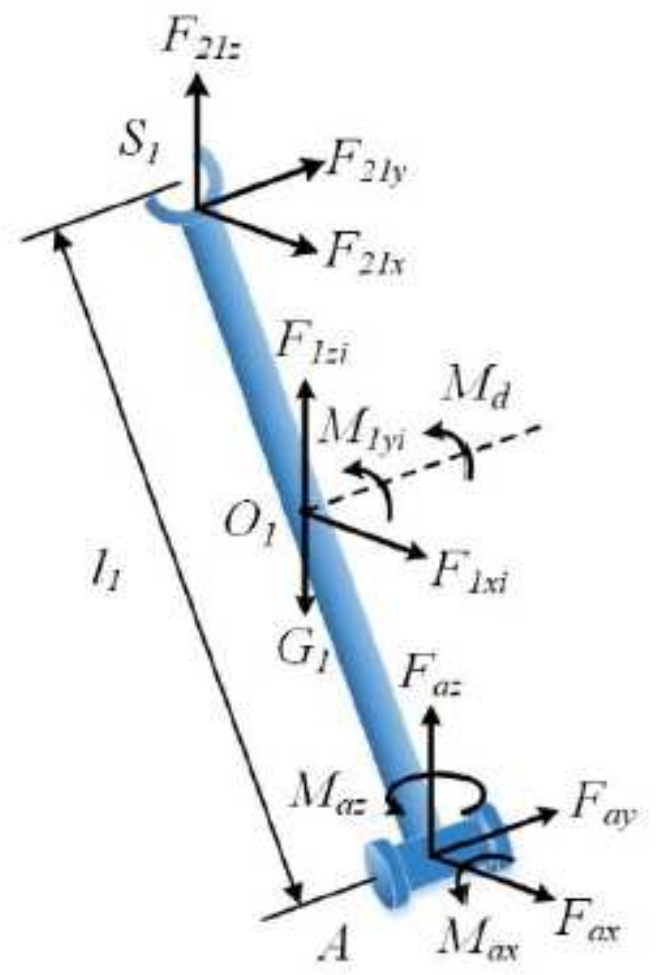

\section{Figure 3}

Schematic of the forces on the rod 1

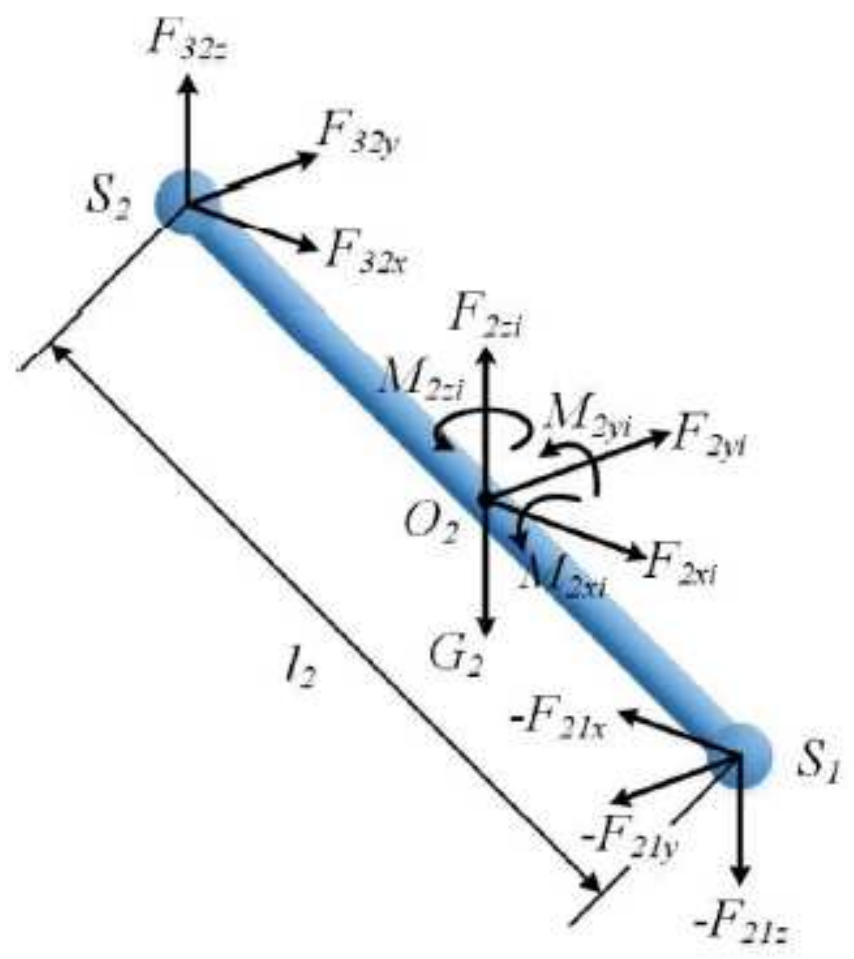


Figure 4

Schematic of the forces on the rod 2

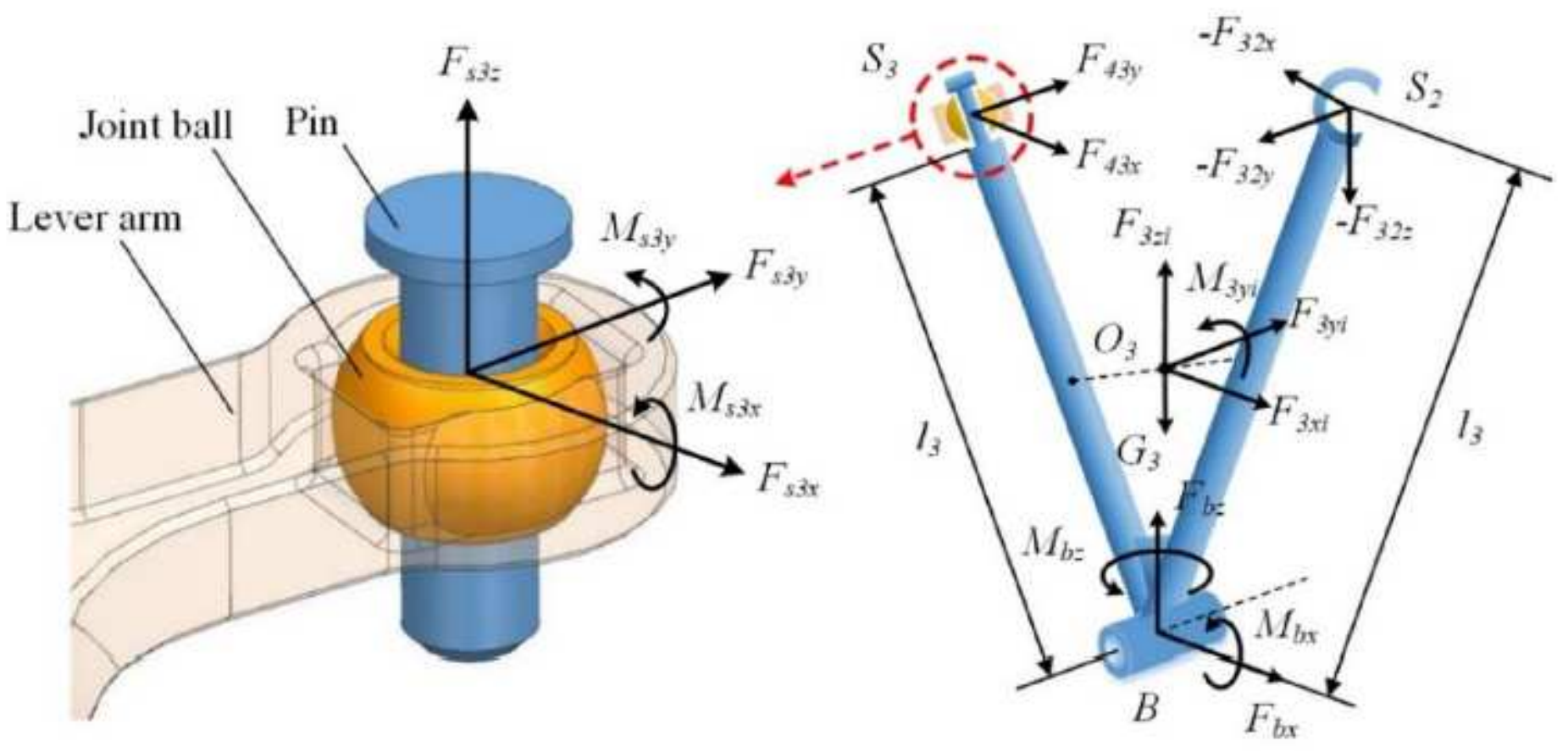

Figure 5

Schematic of the forces on the rod 3

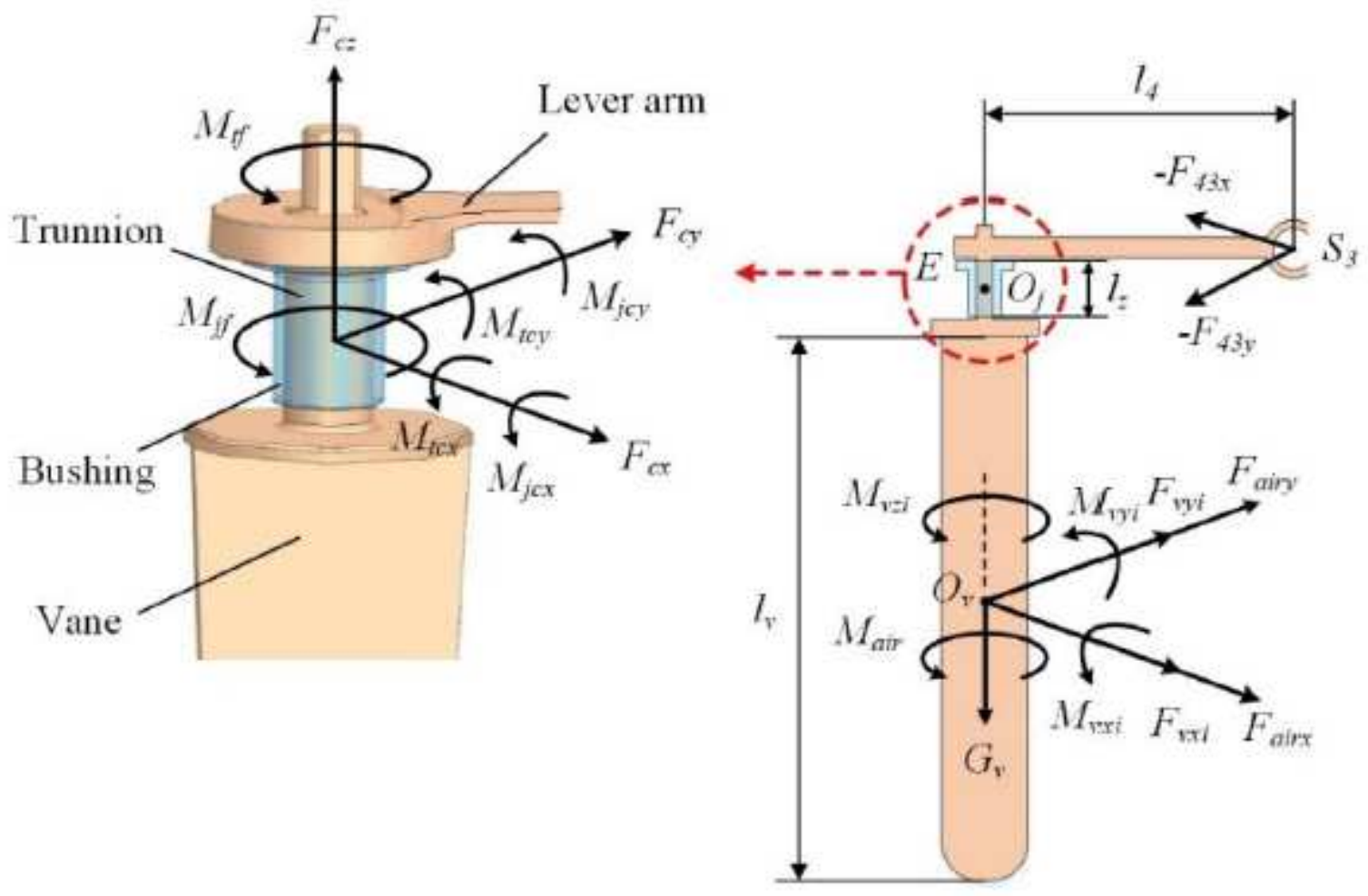


Figure 6

Schematic of the forces on the lever arm and the vane

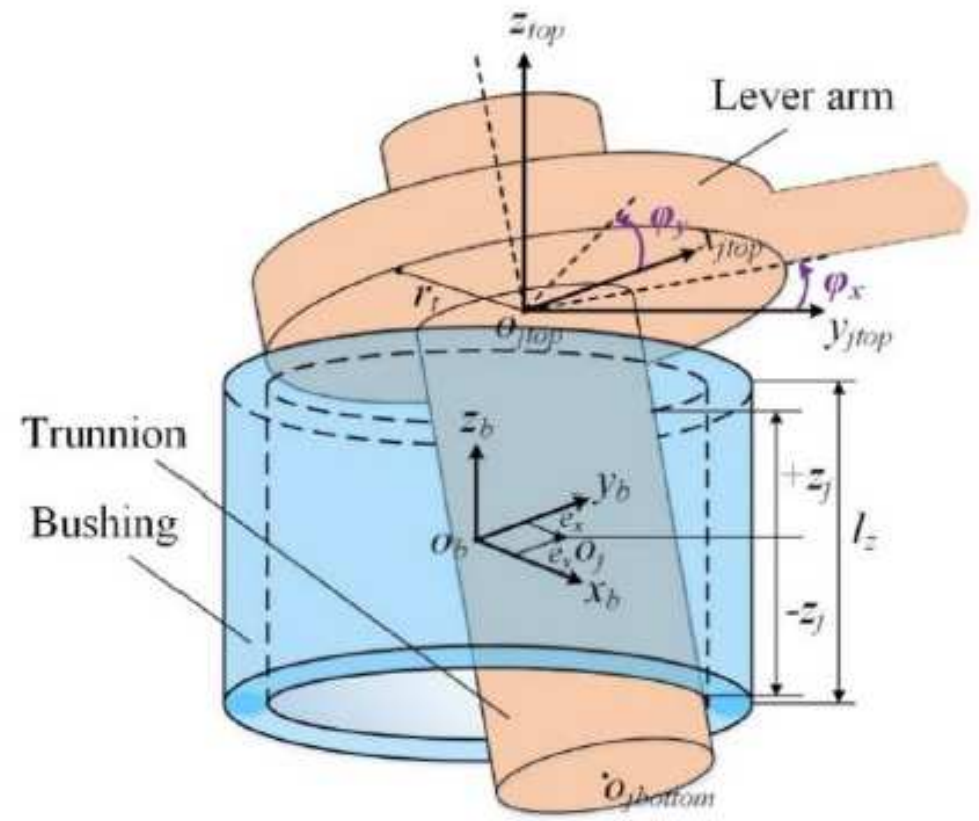

(a)

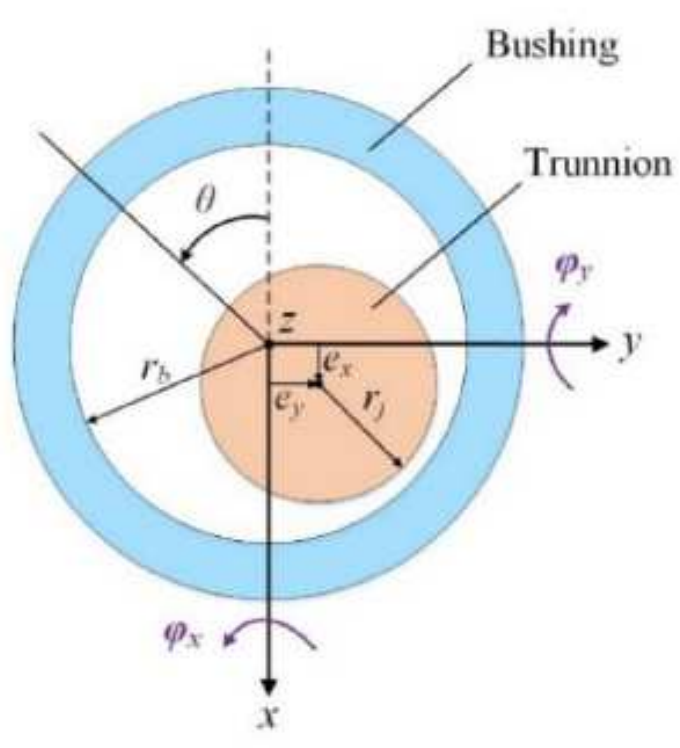

(b)

\section{Figure 7}

Schematic diagram of attitude of the trunnion in the bushing

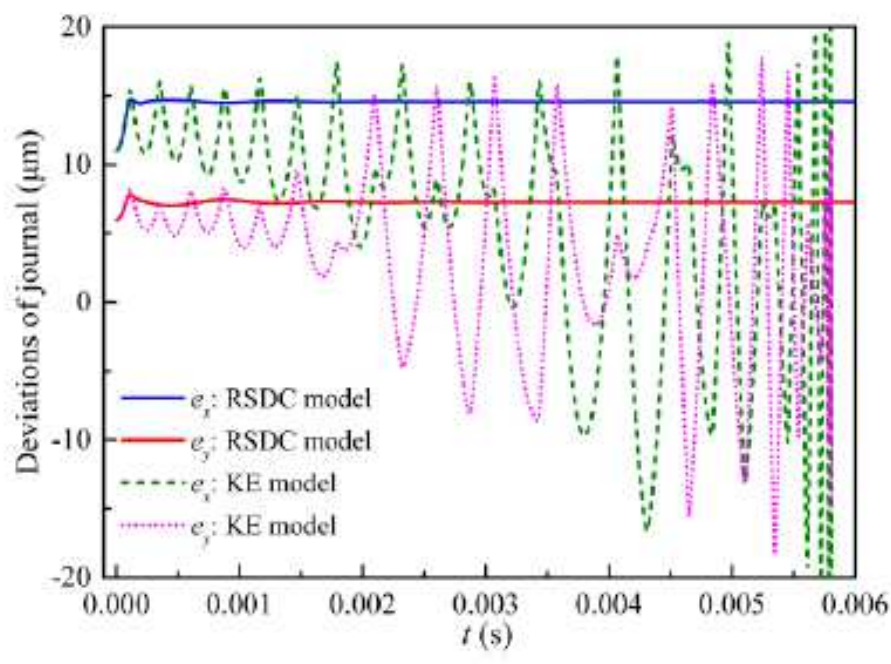

(a)

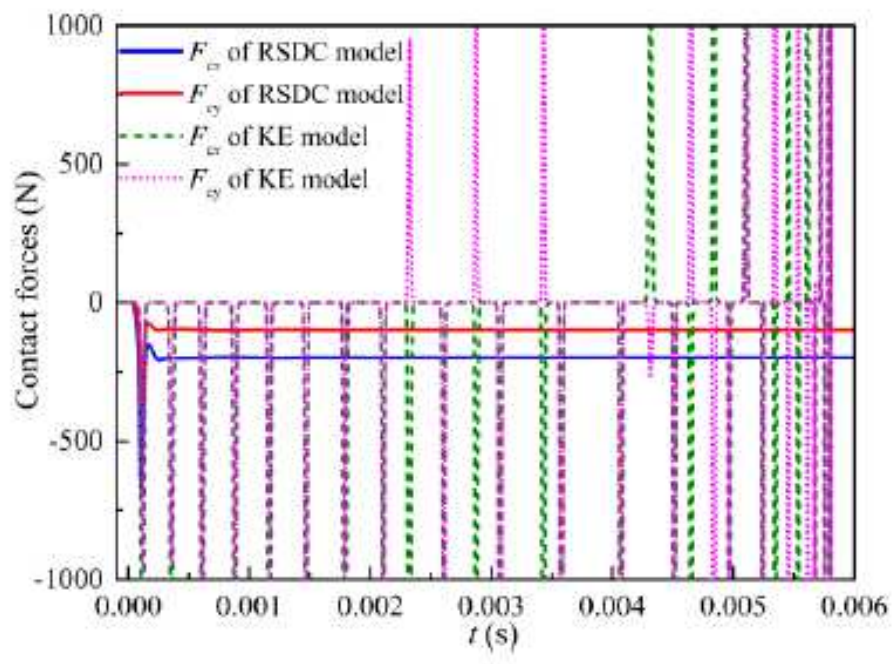

(b)

Figure 8

Journal bearing with dry friction: (a) deviations of journal, (b) contact forces 


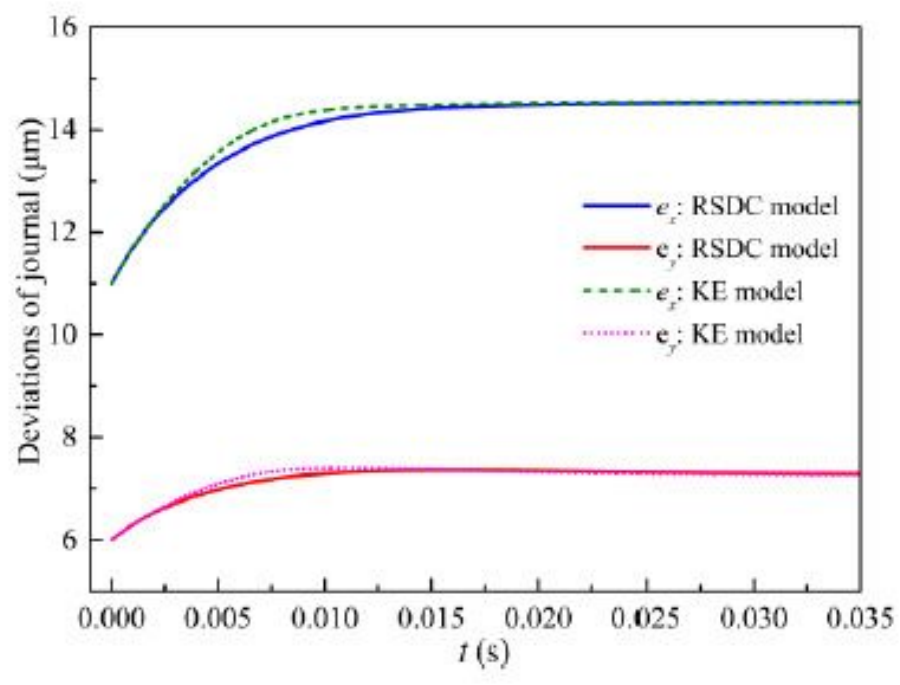

(a)

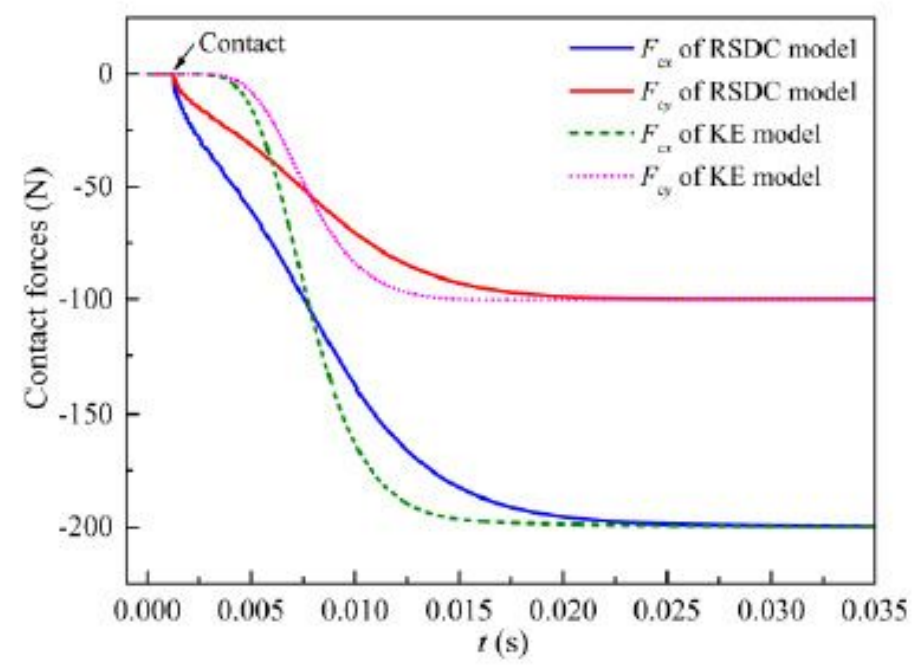

(c)

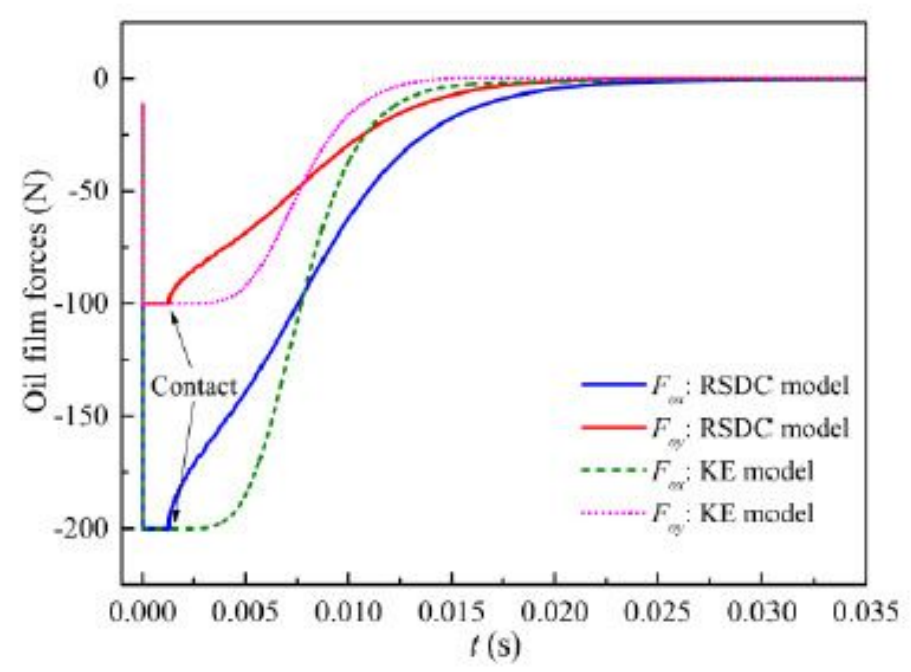

(b)

\section{Figure 9}

Journal bearing with oil lubrication: (a) deviations of journal (b) oil film forces, (b) contact forces 


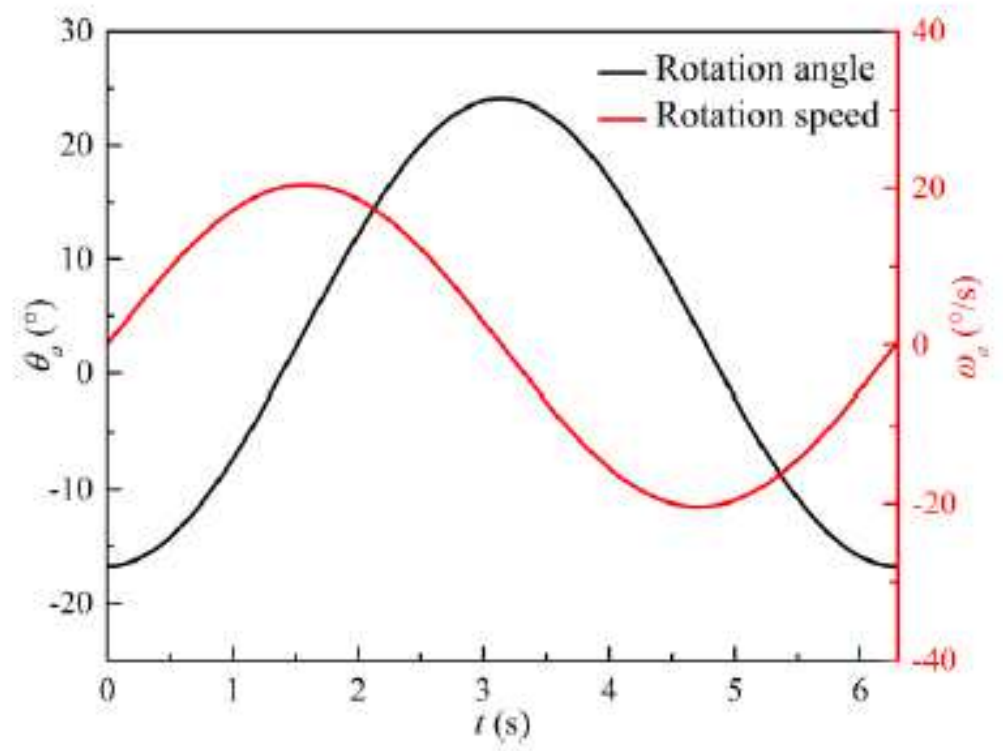

Figure 10

Driving angle and speed of the crank

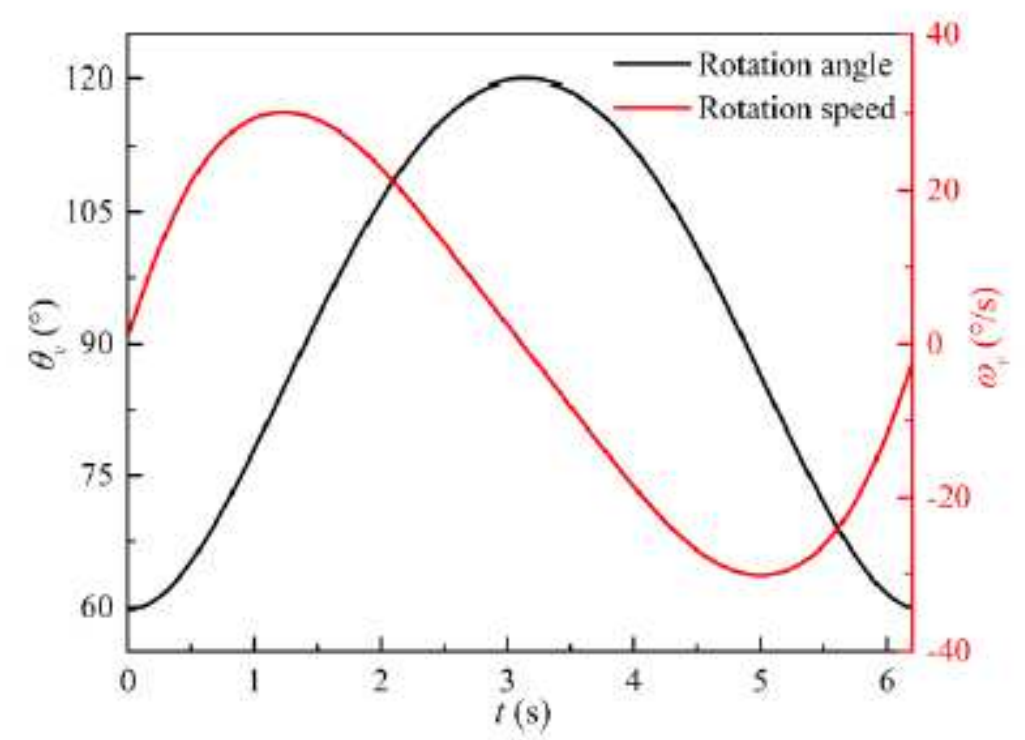

Figure 11

Rotation angle and speed of the vane 


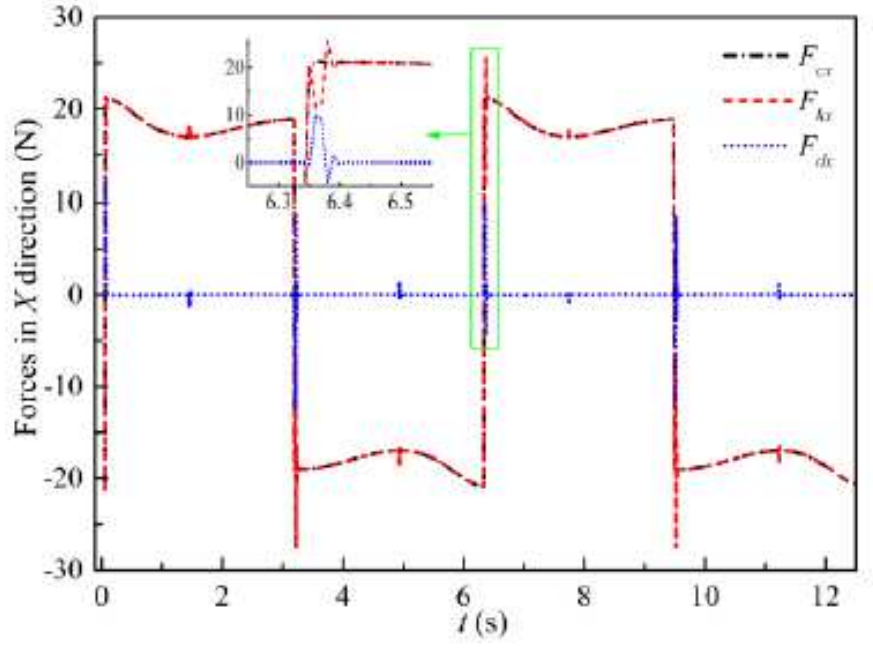

(a)

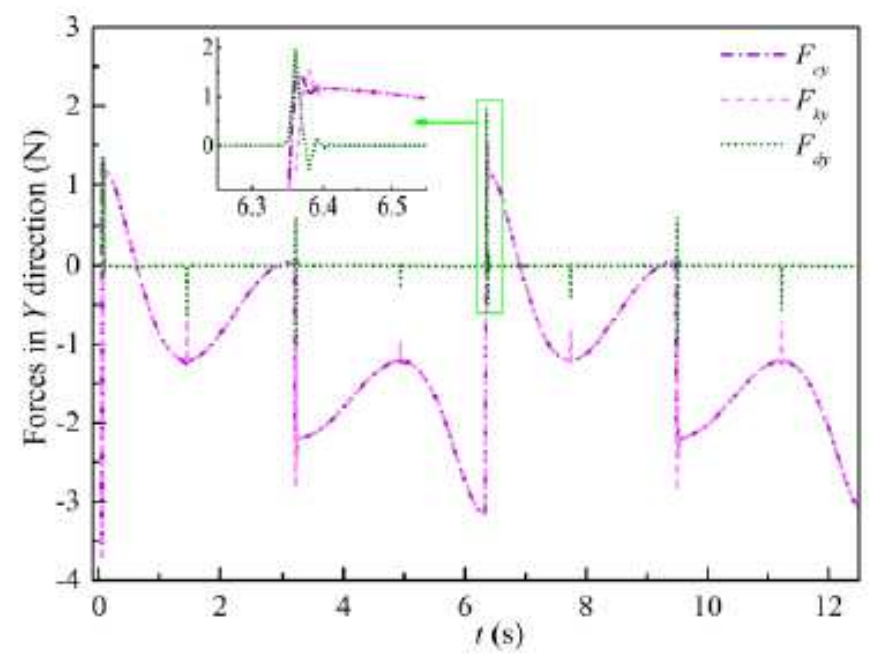

(b)

Figure 12

Contact, rigid and damping forces acting on the trunnion: (a) $X$ direction, (b) $Y$ direction

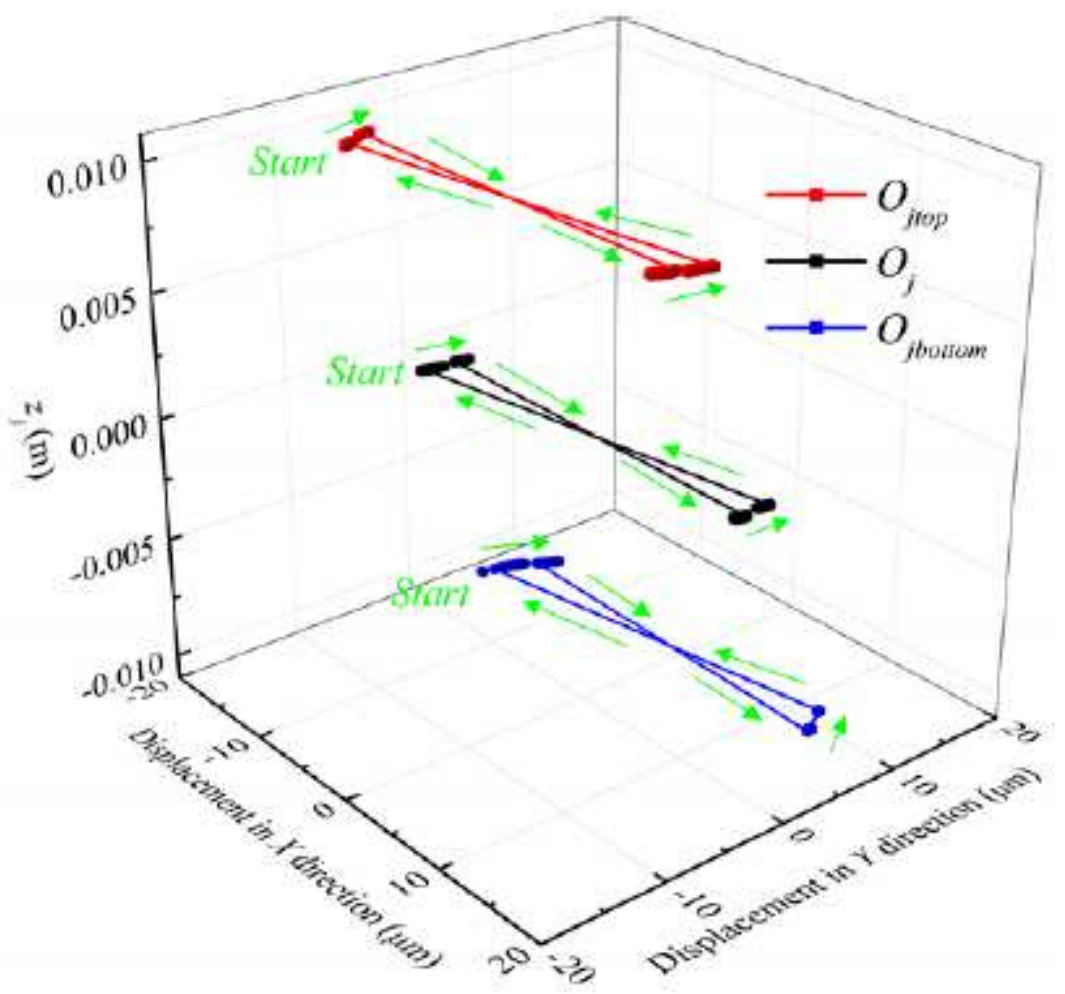

Figure 13

Trajectory of the trunnion 


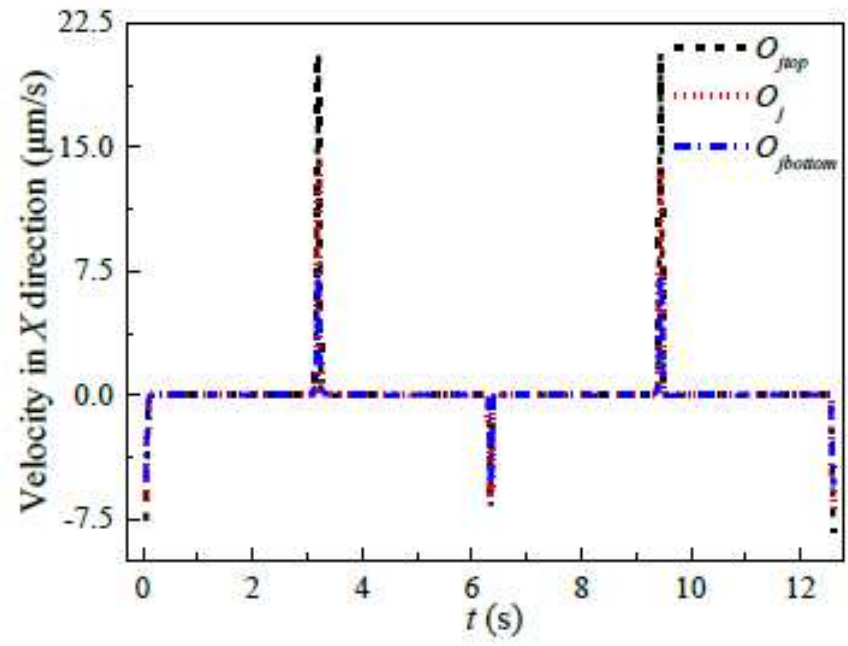

(a)

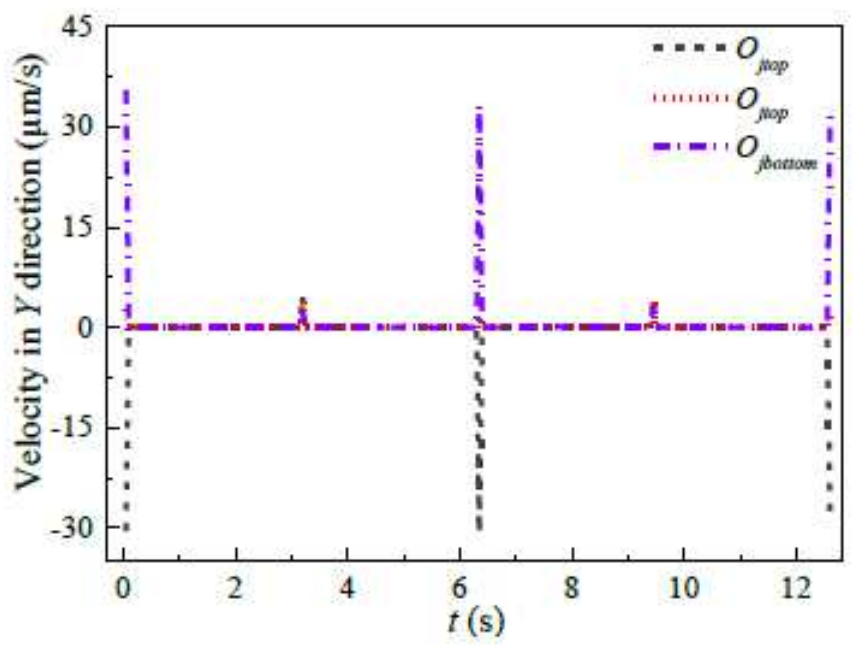

(b)

\section{Figure 14}

Velocity of the three centers of the trunnion in (a) $X$ direction and (b) $Y$ direction 


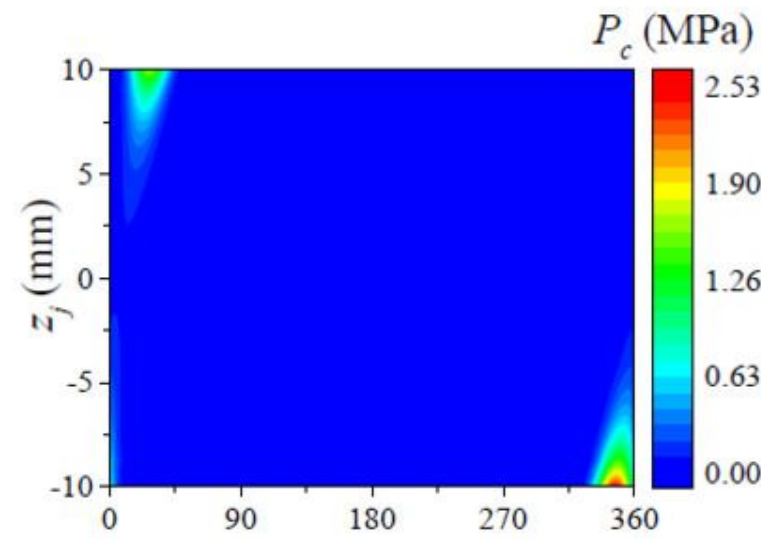

(a)

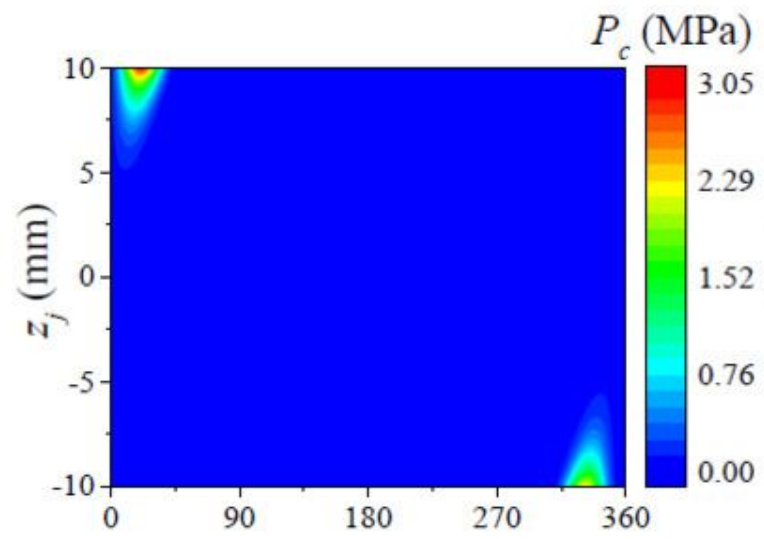

(c)

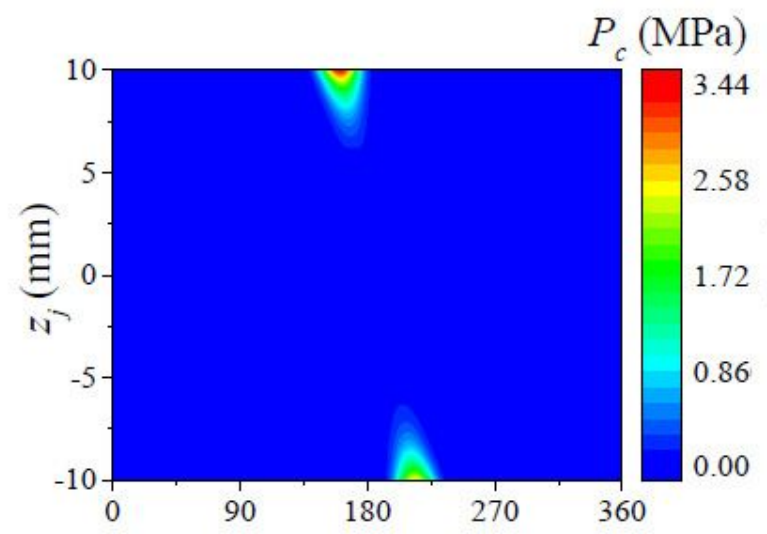

(e)

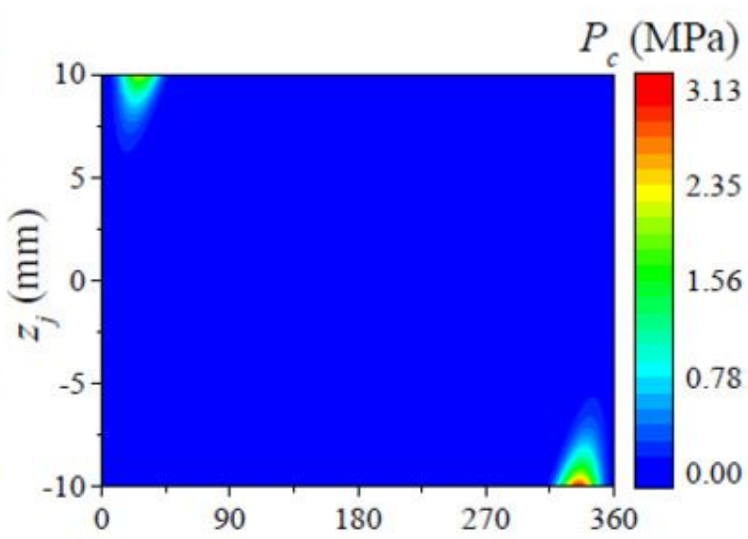

(b)

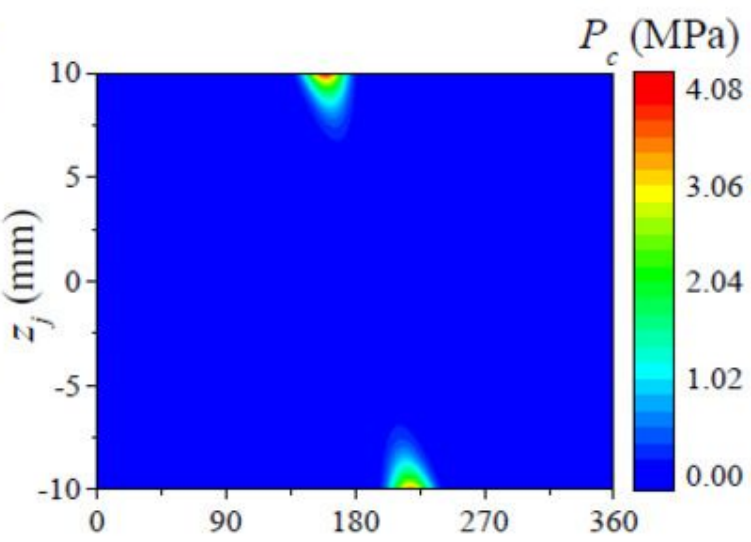

(d)

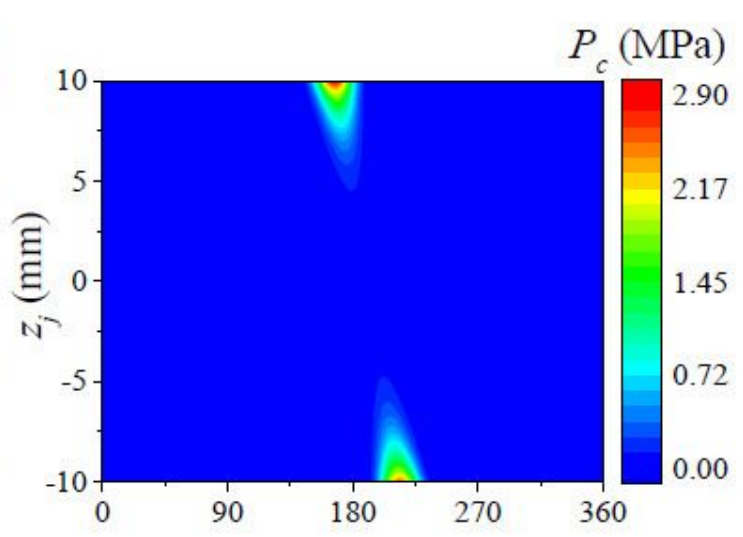

(f)

Circumferential position $\left({ }^{\circ}\right)$

\section{Figure 15}

The contact pressure distribution of the vane at different positions: (a) $60^{\circ}$, (b) $90^{\circ}$, (c) $120^{\circ}$ in forward rotation, (d) $120^{\circ}$, (e) $90^{\circ}$, (f) $60^{\circ}$ in reverse rotation 


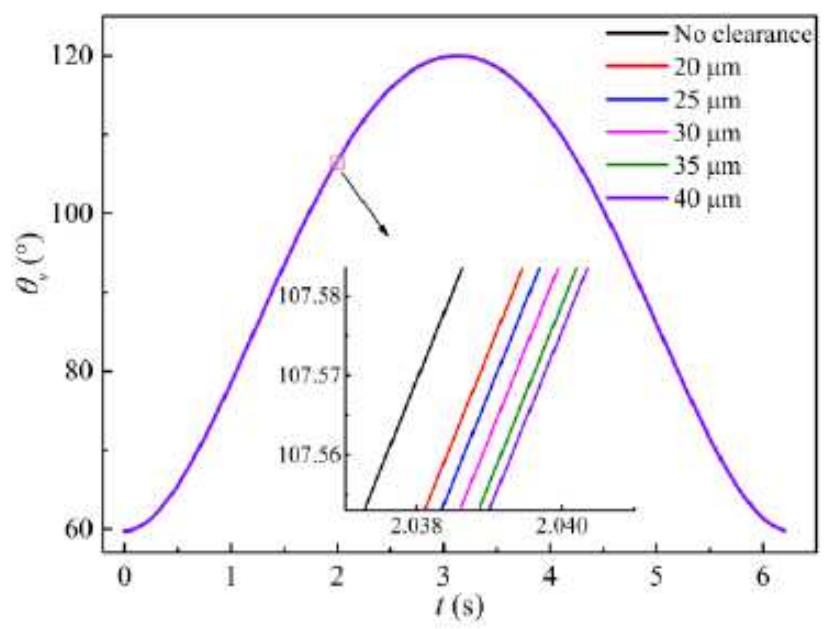

(a)

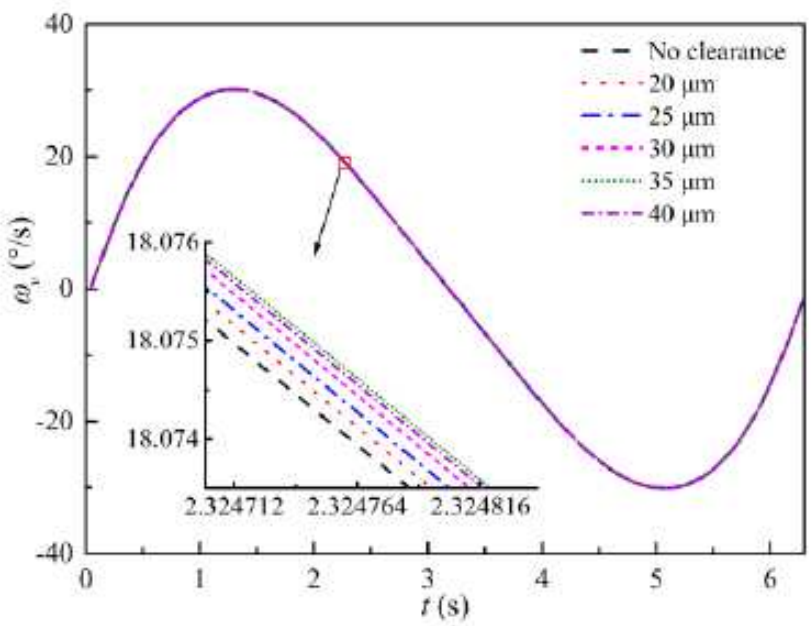

(b)

\section{Figure 16}

Motion state of the vane with different clearance sizes: (a) rotation angle, (b) rotation velocity 


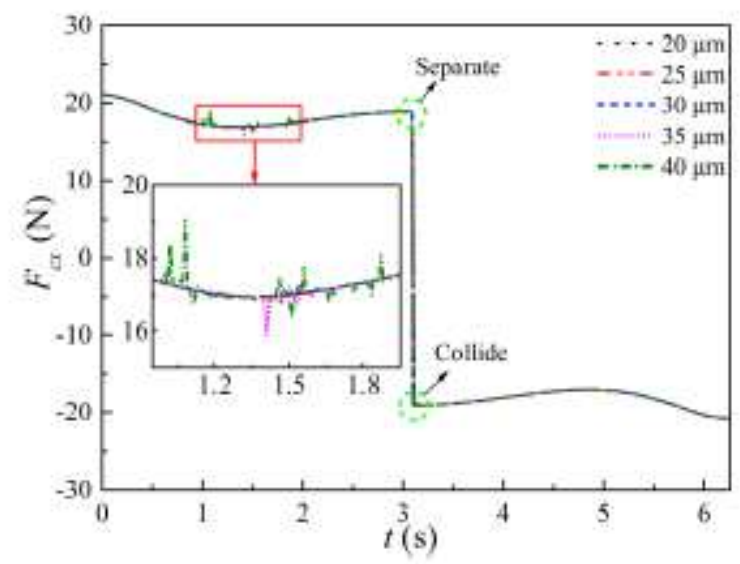

(a)

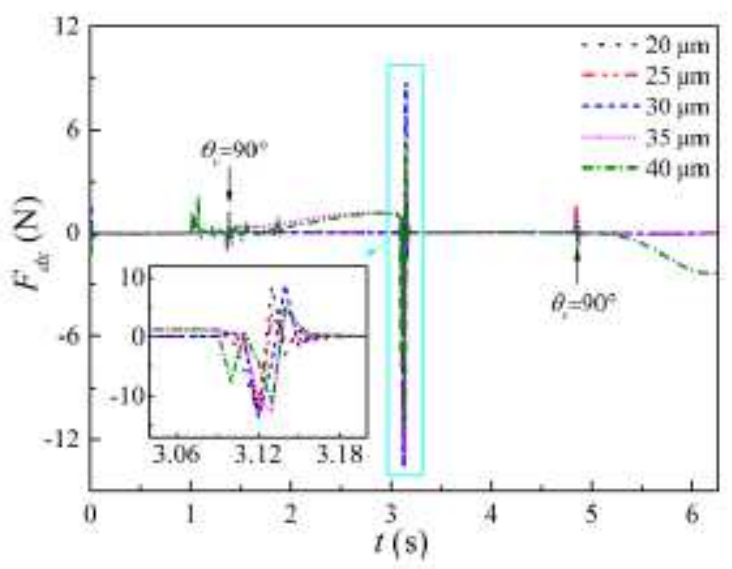

(c)

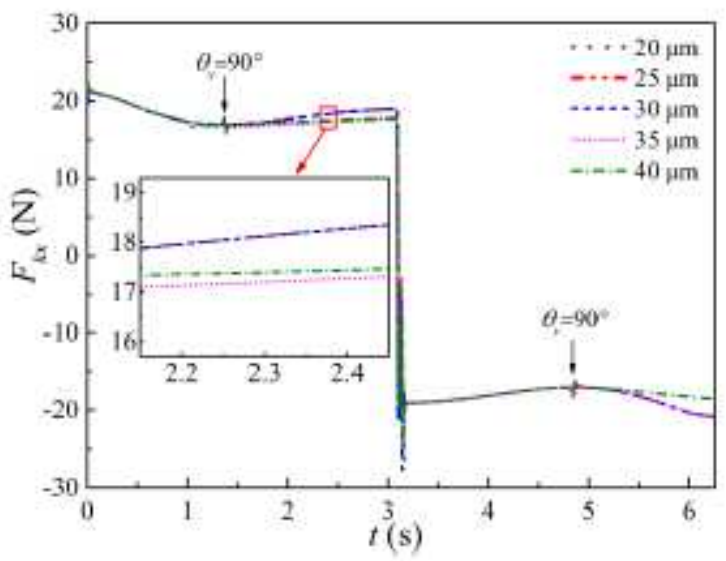

(e)

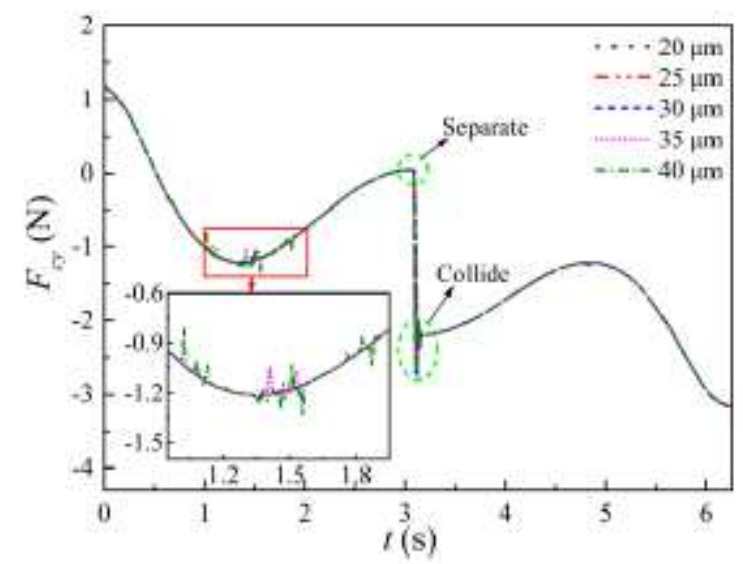

(b)

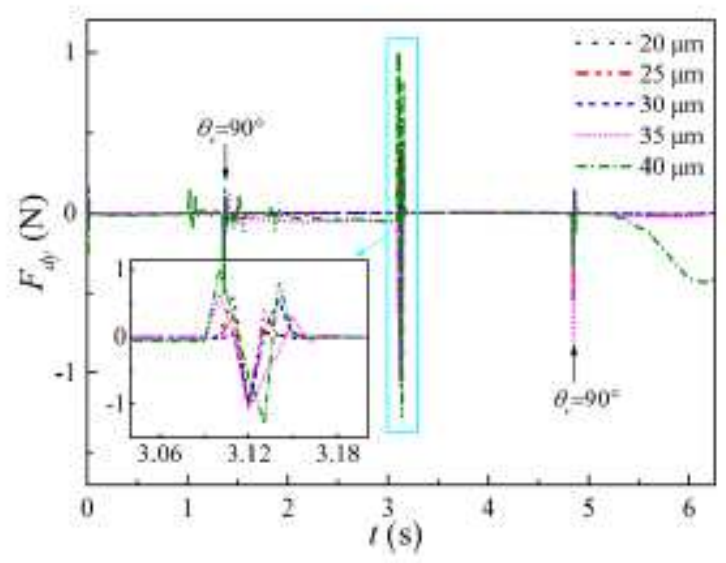

(d)

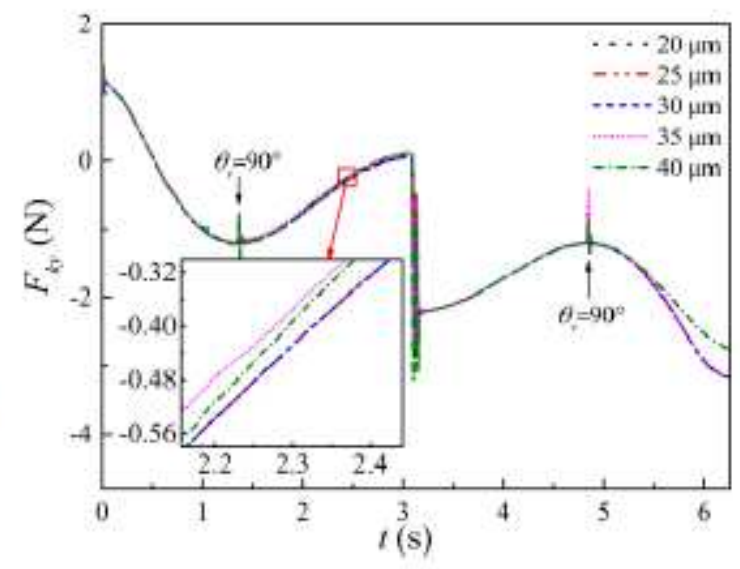

(f)

Figure 17

Variation of forces with different clearance sizes: (a) and (b) for contact forces, (c) and (d) for damping forces, (e) and (f) for rigid forces 


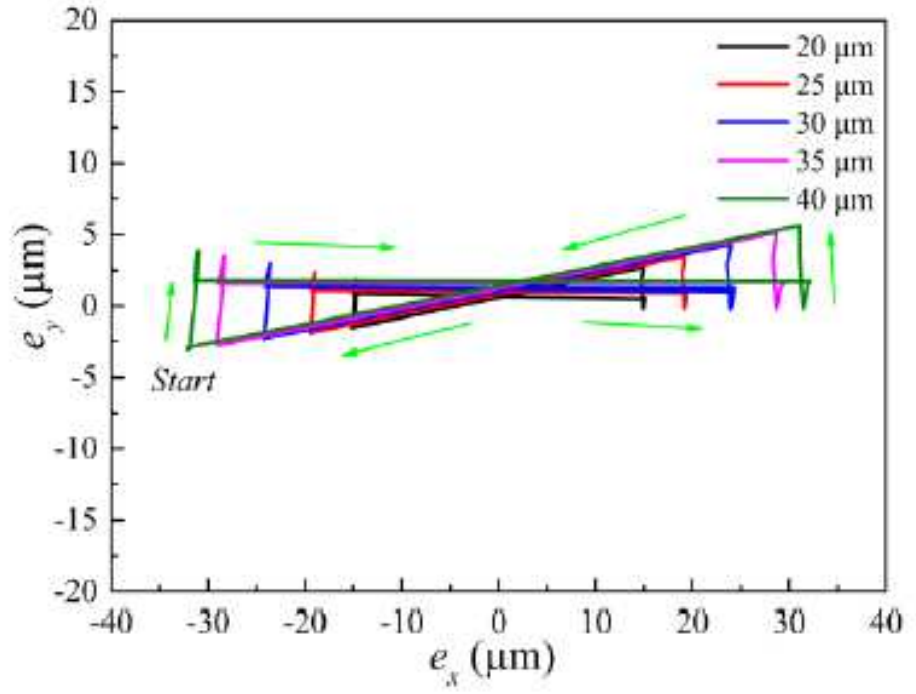

(a)

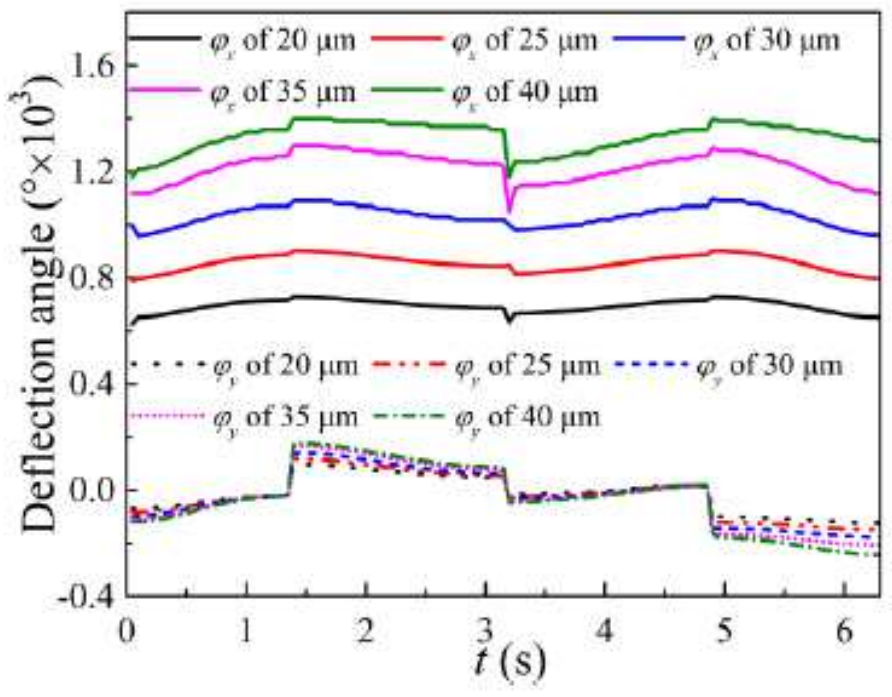

(b)

\section{Figure 18}

Attitude of the trunnion center with different clearance sizes: (a) trajectory, (b) deflection angle 


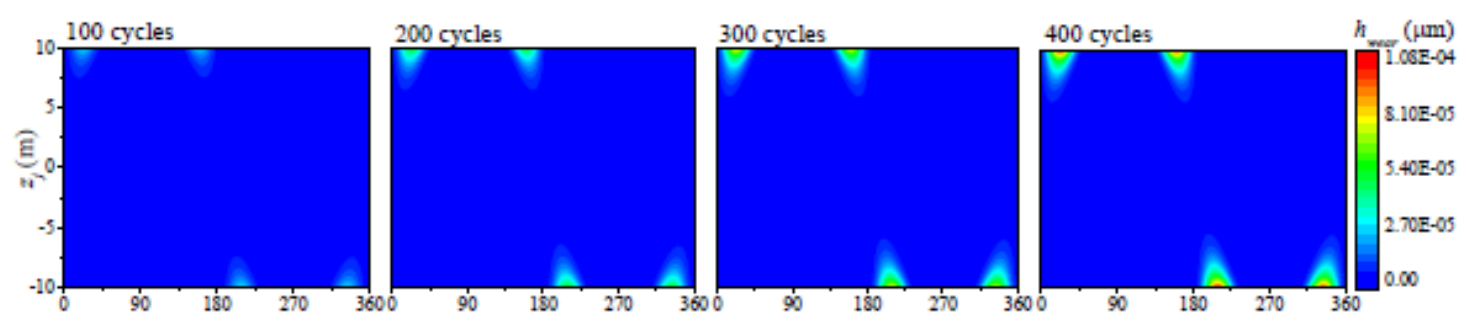

(a)

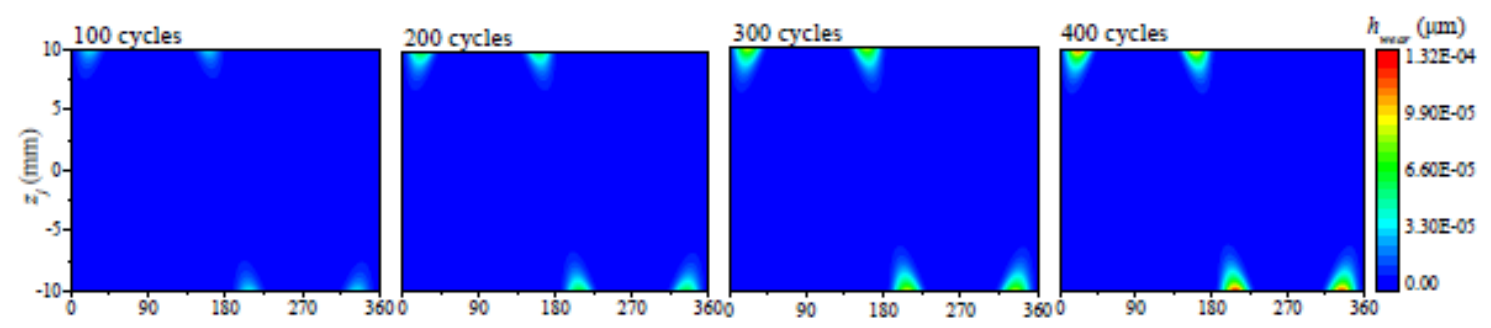

(b)

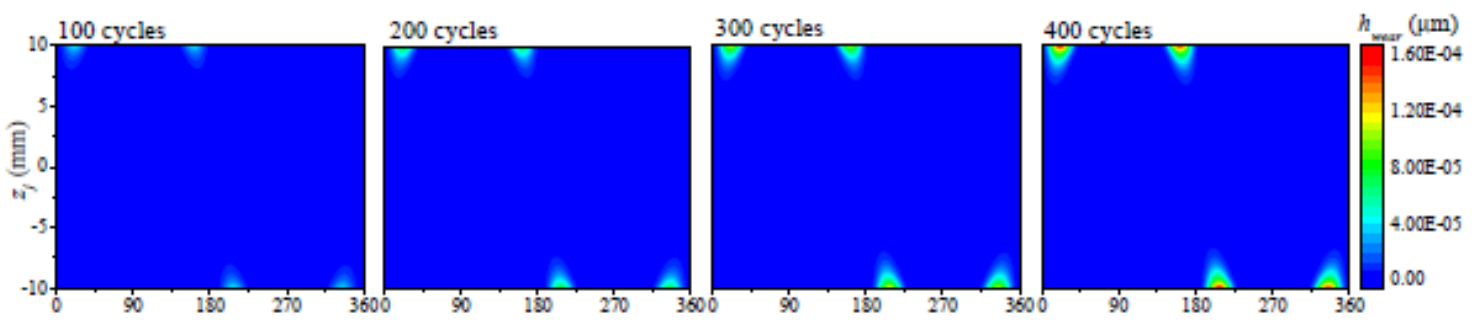

(c)

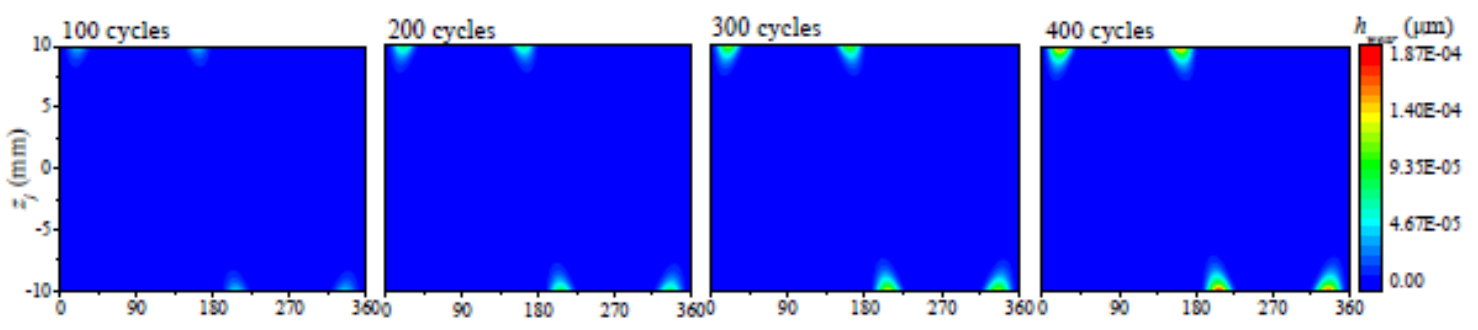

(d)

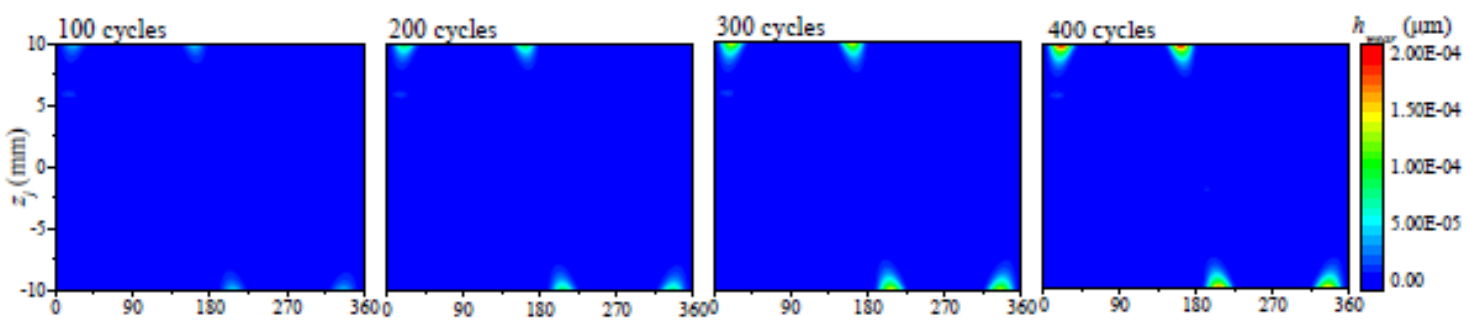

(e)

Circumferential position $\left({ }^{\circ}\right)$

\section{Figure 19}

Wear depth of the bushing with different clearance sizes: clearance = (a) $20 \mu \mathrm{m},(b) 25 \mu \mathrm{m},(\mathrm{c}) 30 \mu \mathrm{m},(\mathrm{d})$ $35 \mu \mathrm{m},(\mathrm{e}) 40 \mu \mathrm{m}$ 